Orniversità degli. Pudidi. Firenne

DIPARTIMENTO DI MECCANICA E TECNOLOGIE INDUSTRIALI

TESI DI DOTTORATO DI RICERCA IN PROGETTO E COSTRUZIONE DI MACCHINE

XXI CICLO

\title{
STRESS ANALYSIS OF A CVT BELT TRANSMISSION
}

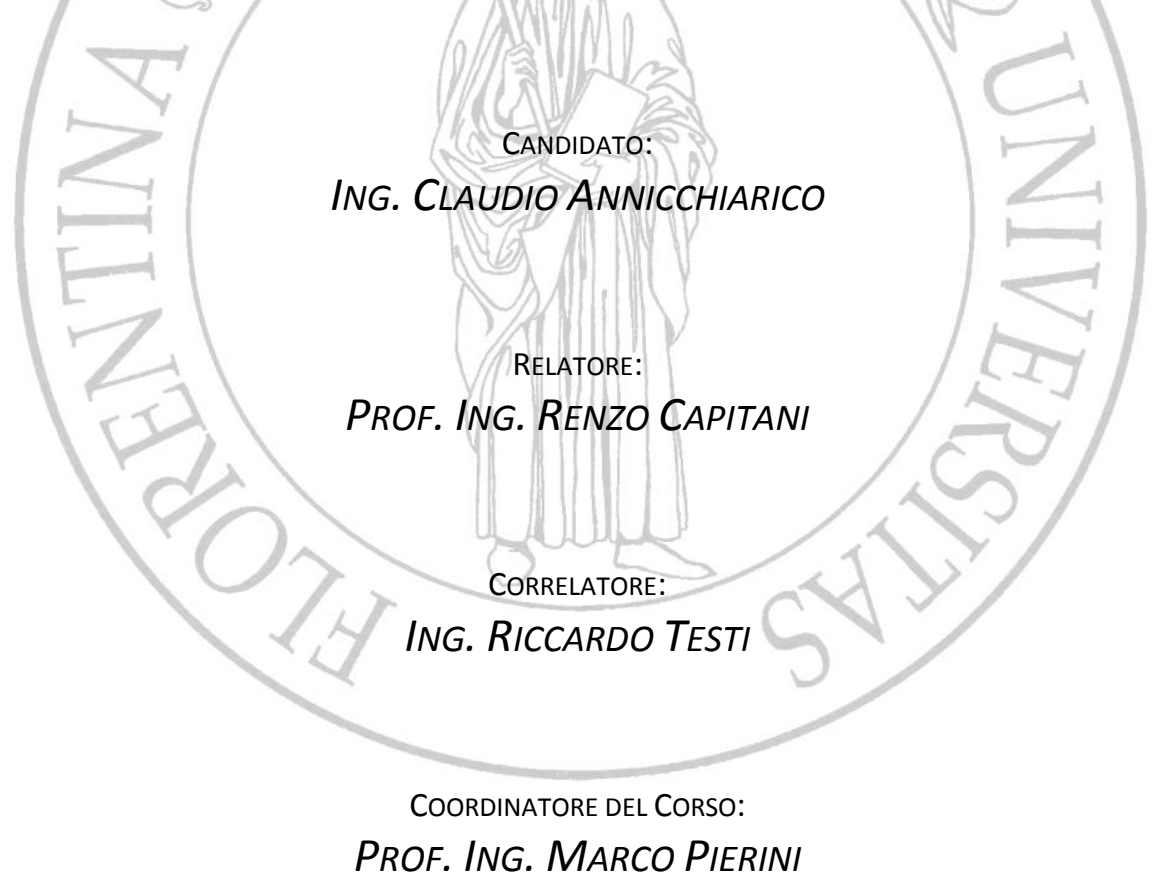



A IRENE, Silvana E CiRo

IMAGINATION, NOT INTELLIGENCE, MADE US HUMAN TERRY PRATCHETT 



\section{Abstract}

In this work we have analyzed the stress field acting on the CVT (Continuously Variable Transmission) used by the Piaggio Company in high displacement scooters. In detail, we have focused on the belt and on the driven pulley, that are the critical parts of the assembly. The analysis has been conducted with a full MultiBody model of the mechanism combined with the Finite Element analysis of both the belt and the pulley. The output data so obtained have been used in a fatigue analysis in order to define the reliability of these parts.

The belt durability has been computed with the Miner approach, using the fatigue data supplied by the producer. This analysis has shown that the estimated lifetime of the belt under the Piaggio standard test at the engine bench is lower than 70 hours.

As far as it concerns the pulley, we have evaluated the stress field produced by both the manufacturing process and the working conditions. We have then compared the resulting stress field the fatigue strength of the pulley material, that has been evaluated with a staircase approach based on 15 specimens milled from a die cast part. This analysis has shown that the stress field acting on the flange is lower than the fatigue strength of the material. Considering the standard deviation of the staircase measurements, the reliability of the flange with respect to an infinite lifetime expectation is $75 \%$. 



\section{Contents}

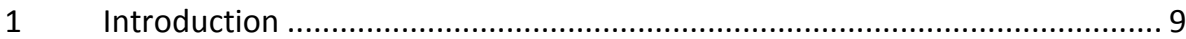

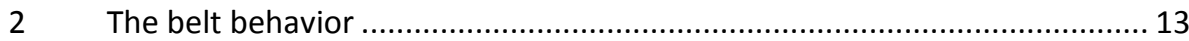

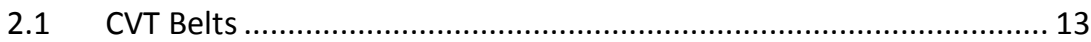

2.2 The belt proportioning system ......................................................... 15

2.3 The Finite Element modeling of the belt .......................................... 18

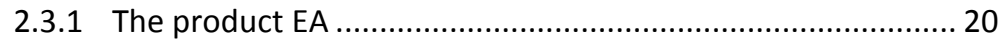

2.3.2 The product EJ ............................................................ 22

2.3.3 The stiffness matrix .............................................................. 24

2.4 Another possible use of the belt FE model ....................................... 25

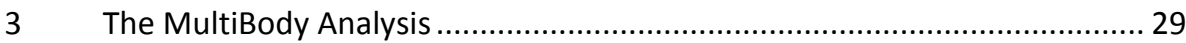

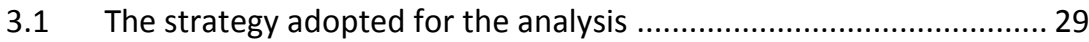

3.2 An outline of the CVT Model.............................................................. 31

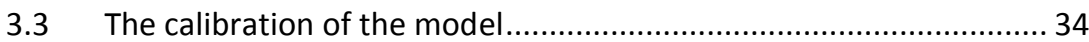

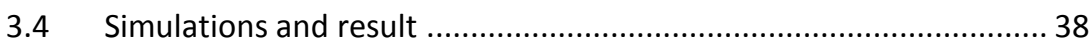

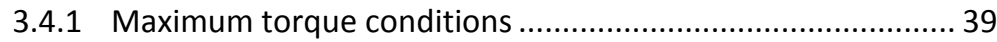

3.4.2 Maximum power conditions ................................................ 45

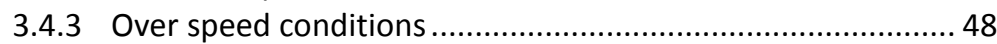

3.4.4 Lifetime of the belt ............................................................... 51

$4 \quad$ The residual stress acting on the pulleys ................................................... 53

4.1 The riveting process .................................................................... 53

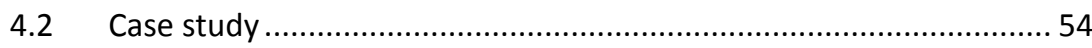




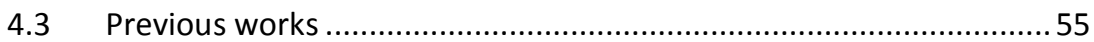

4.4 The complete FE model of the assembly ......................................60

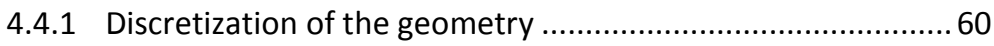

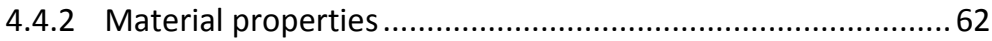

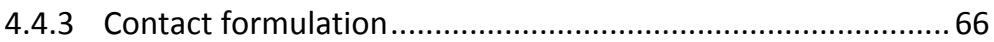

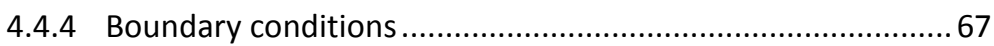

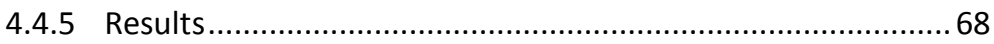

$5 \quad$ The fatigue strength of the flange material .............................................. 81

$5.1 \quad$ Material and process matching .................................................... 81

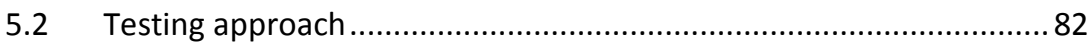

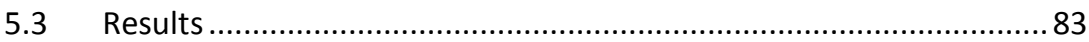

$6 \quad$ The stress field acting on the pulleys in working conditions ....................... 85

$6.1 \quad$ The fatigue calculation approach ................................................ 85

6.2 The FE analysis of the working conditions .................................... 88

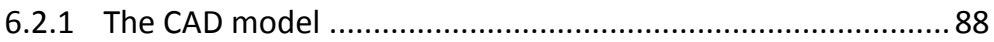

6.2.2 The FE model..................................................................... 90

6.2.3 The first load case: the riveted flange................................. 92

6.2.4 The maximum torque conditions........................................ 94

6.2.5 The maximum power conditions ......................................... 97

6.2.6 The over speed conditions ............................................. 100

6.3 The reliability of the flange ........................................................ 103

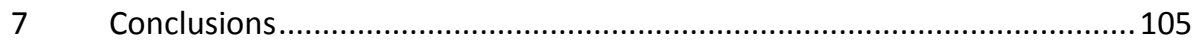

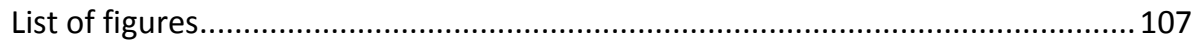

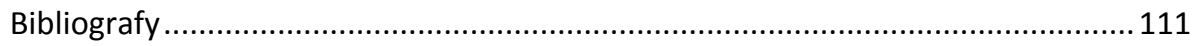




\section{Introduction}

The globalization has considerably widen the supply spectrum in the Automotive field, destabilizing the past equilibriums and generating a more competitive market. If in the past the market was split between American, European, Japanese and Korean societies, in the last decades new producers, mainly Chinese and Indian, have become more and more competitive even in the global market [1]. Moreover, the geographic reassembly of the global trades and the increasing in the global per capita income have contributed to build up the demand of vehicles in the Developing Countries, generating new market fields and new competitors [2]. The consequent increasing in supply has led to a new consumer profile, more and more mindful to the product quality, in terms of both technical features, like performances and consumptions, and perceived quality, like assembly accuracy and noise.

The increasing competitiveness of the Automotive market has imposed an important reduction in time to market, in order to adapt the project specifications to the current expectations of the customer. This tendency has led to a drastic reduction in the time dedicated to the development stage, from the approval of the style sketch to the introduction in the market. Therefore, in a modern scheme the approval of drawings and the production of the parts must be as quick as possible, but the reduced time spent in the design phase cannot imply a reduction in robustness of the choices, since the errors done in this phase have a great effect on the product cost [3]: some authors stated that $70 \%$ of the cost of a product, considering its whole life cycle, is decided in the design phase [4], [5]. 


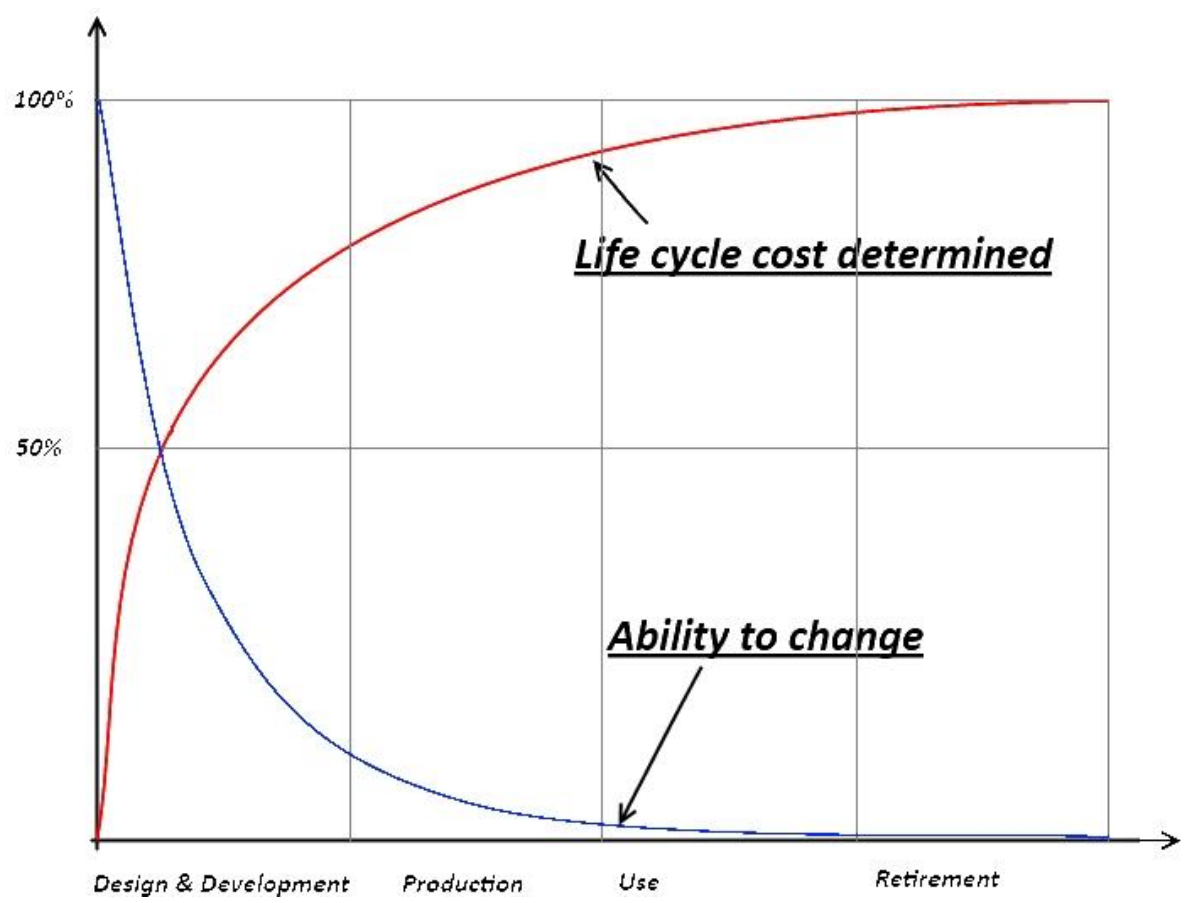

Figure 1.1: Cost build up in the life cycle of a product

For instance, a design error could lead to undersized part in the product; if this issue is not noticed before the production phase, it is often necessary to modify the material of that part or its production process, with a consequent increase in costs. To avoid this kind of issues, it is necessary to understand the stress level in the early phase of the design process, in order to simplify the selection of materials and technologies, and so executing the CAD prototyping on the basis of the manufacturing process needs.

In this work we have analyzed the stress field acting on a continuously variable transmission (CVT) mechanism used on the high displacement scooters produced by the Piaggio Company, trying to extrapolate the most important results of the analysis with the aim of providing the designers with some guidelines useful to reduce the design error occurrence. This work could serve as a base to compile a new proportioning method, that should be based on the whole stress history of each part of the assembly, computed with the aid of some numerical tools.

The CVT assembly is generally composed by a V-Belt and two pulleys with variable pitch diameter. As shown in Figure 1.2, the drive pulley is composed of two flanges (the half pulleys), one fixed on the shaft and the other free to move in the axial direction. 


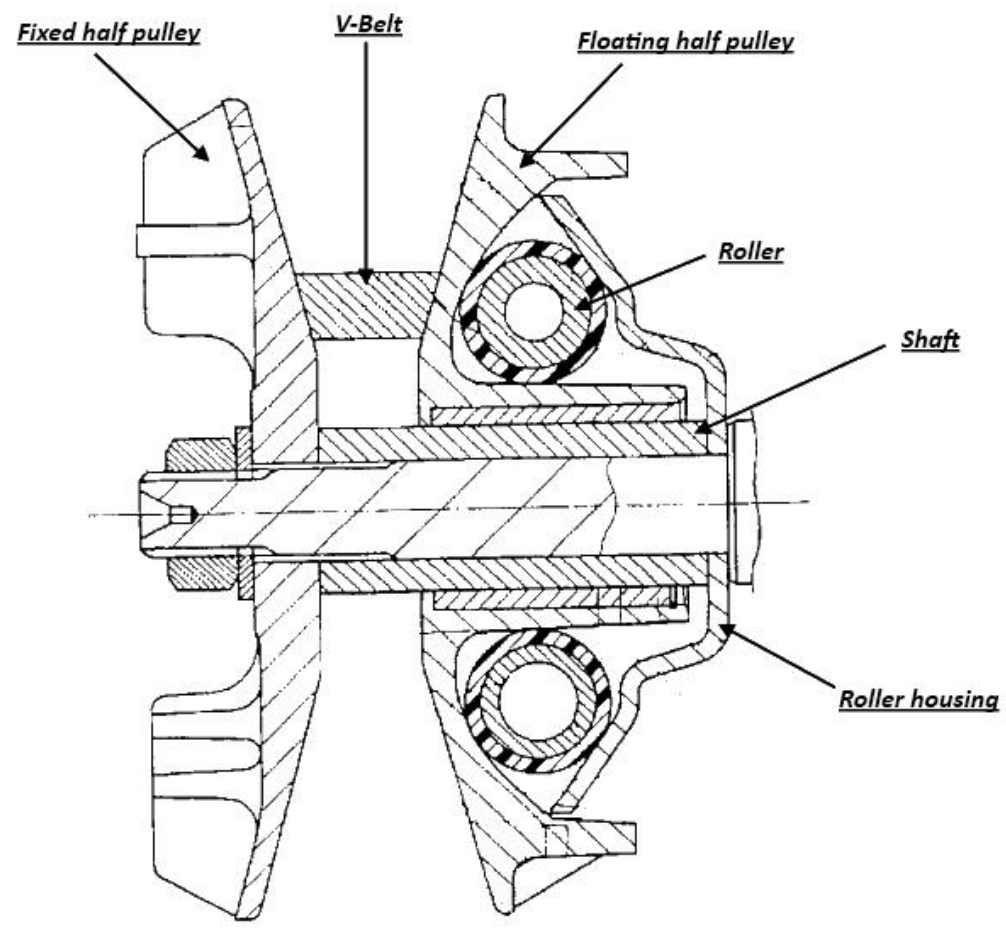

Figure 1.2: CVT working scheme - The drive pulley

On the floating half pulley there are some grooves where the rollers are positioned; these cylindrical bodies move in the radial direction under the effect of the centrifugal force, sliding on the surface of the roller housing. Therefore, the shape of the grooves in the floating half pulley imposes the variation of the wrapping diameter of the V-Belt on the drive pulley as a function of the rotational speed of the shaft. 


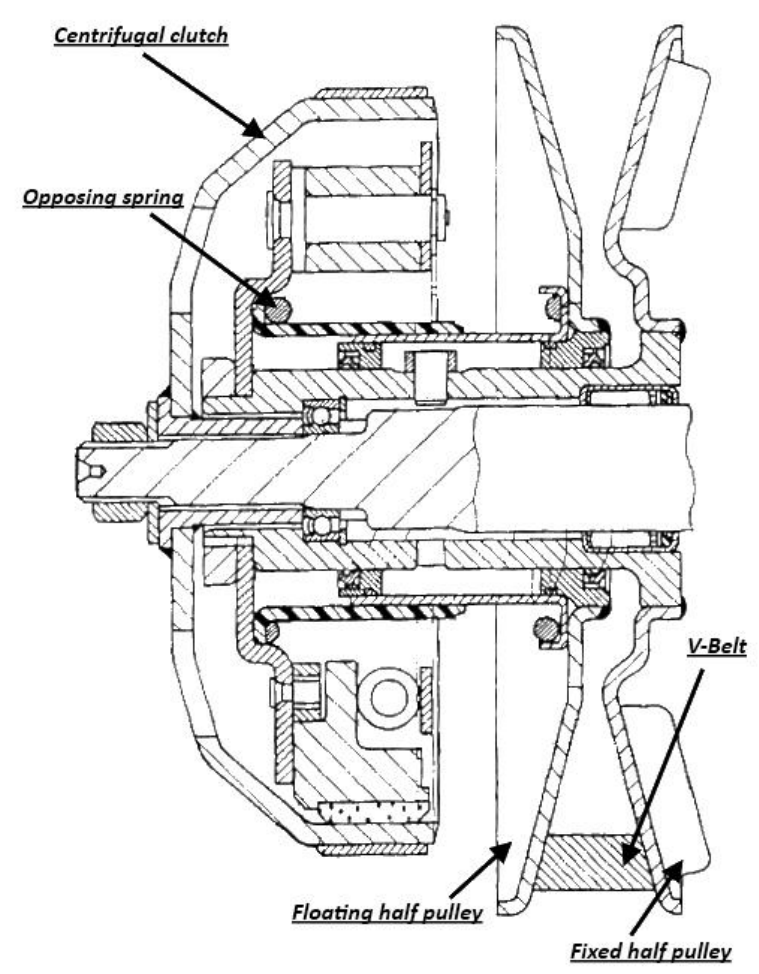

Figure 1.3: CVT working scheme - The driven pulley

Due to the high axial stiffness of the belt (usually made of composite material, with Kevlar plies in an elastomeric matrix), an increment in the drive pitch diameter must be paid by a reduction in the driven pitch diameter. As shown in Figure 1.3, even the driven pulley is realized by two flanges, one fixed to the shaft and the other axially free. The contact with the belt is ensured by the action of an opposing spring, installed between the floating half pulley and a flange joined to the shaft. In the same Figure is shown the centrifugal clutch joined to the shaft.

This system is subdued to high stresses connected to the contact pressure that builds up between the pulleys and the side wall of the V-Belt. Unlike other works [6], we have analyzed the behavior of the mechanism even in unsteady state, i.e. during the variation of the transmission ratio, highlighting some issues often omitted in the design of the CVT. 


\section{The belt behavior}

\subsection{CVT Belts}

The typical layout of the CVT used on motor scooters contemplate a trapezoidal belt (also called V-belt) and two expandable pulleys. The belt is usually composed of a core that represents the load-carrying element of the belt, and of two structures that realize the external geometry of the belt.

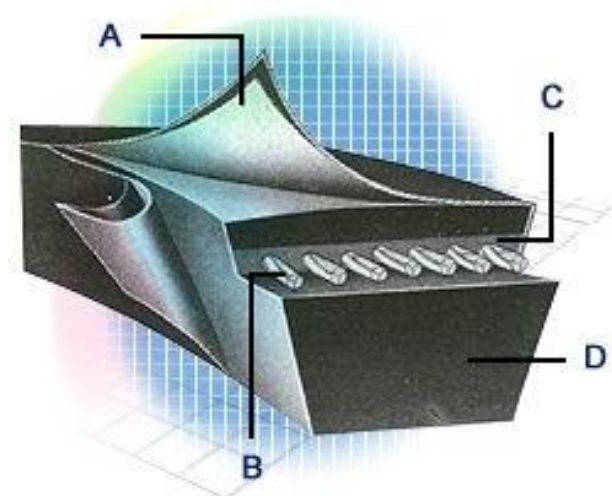

Figure 2.1: Typical CVT belt cross section

In reference to Figure 2.1, the core of the belt (indicated by the letter " $\mathrm{B}$ ") is usually composed of a series of plies made of stiff material, like Kevlar or steel, and sometimes disposed on multiple layers in order to ensure the requested tensile strength of the belt, providing a high fatigue life and a good resistance to elongation. Those cords are bonded in their position with an epoxidic compound (letter " $C$ "). The upper and lower sides of the belt (letter " $D$ ") are composed of two elastomeric pads reinforced with short fibers of high stiffness material; their role is to transmit the traction force to the pulleys and to avoid the wedging of the belt into the pulleys' groove. In some cases there is a protective cover (letter " $A$ ") to improve the wear resistance of the belt. 
The CVT belts usually show a series of cogs on both the upper and the lower side, to increase the flexibility of the structure and to improve the heat dissipation.

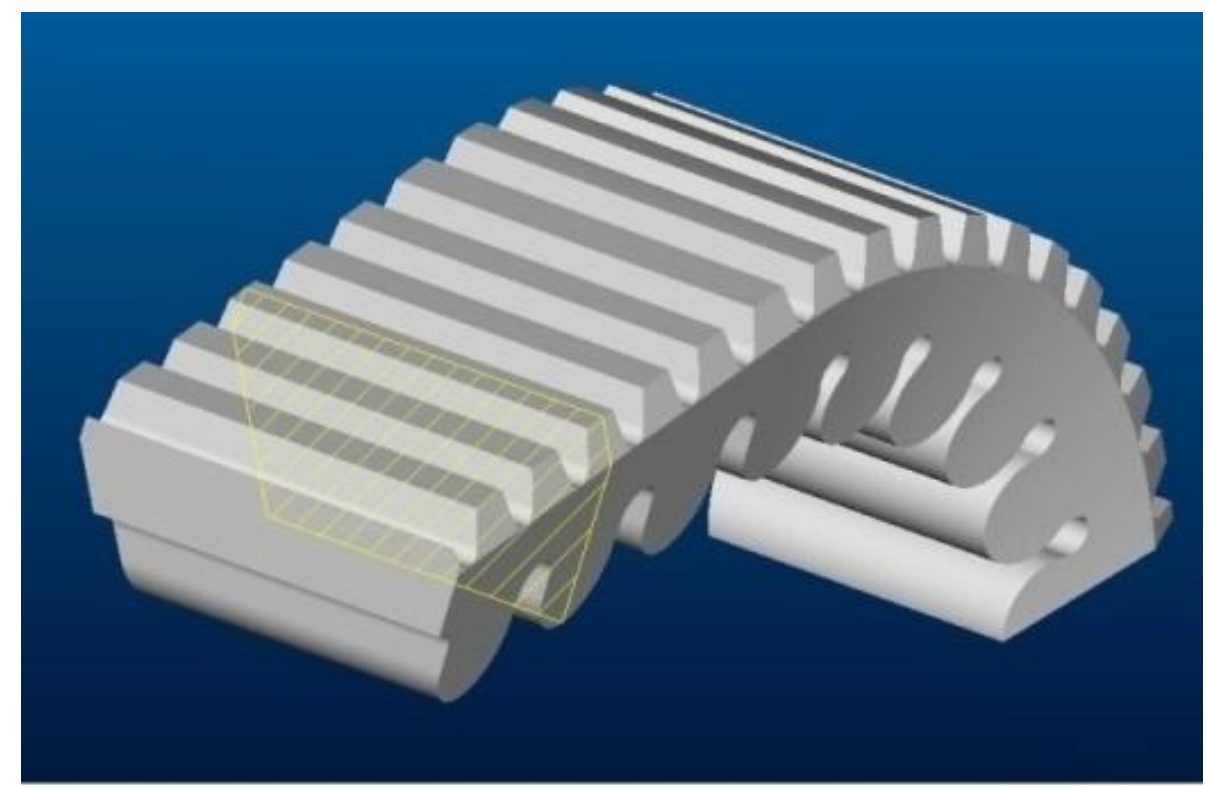

Figure 2.2: Toothed shape of a typical CVT belt 


\subsection{The belt proportioning system}

The main causes of failure for a belt are the breaking of the core, usually anticipated by a crack into the lower elastomeric pad, and the excessive wearing of the sides that brings the belt to wedge into the groove more than expected, also limiting the friction coefficient with the pulleys.

The belt life calculation is usually conducted using the experimental data supplied by the producer that refer to an equivalent flat belt transmission with a "virtual" friction coefficient with the pulleys given by the equation (2.1).

$$
\mu_{v}=\frac{\mu}{\sin (\alpha)}
$$

Where:

- $\quad \mu \quad$ is the actual friction coefficient

- $\mu_{V} \quad$ is the virtual friction coefficient

- $\alpha \quad$ is the half angle of the belt sides.

The typical belt proportioning method for flat belts requires the imposition of the following conditions:

$$
\begin{gathered}
T_{t}<T_{\lim } \\
p<p_{\text {lim }}
\end{gathered}
$$

Where:

$$
\begin{array}{lll}
- & T_{t} & \text { is the belt tension measured in the tight side } \\
- & T_{\lim } & \text { is the maximum tension sustainable by a given belt } \\
- & p & \text { is the pressure acting on the sides of the belt } \\
- & T_{\text {lim }} & \text { is the maximum side pressure sustainable by a given belt. }
\end{array}
$$

The tension is $T_{t}$ computed on the tight side of the belt with the well known equation (2.4).

$$
T_{t}=\frac{C_{m} e^{\mu_{v} \varphi}}{R_{m}\left(e^{\mu_{v} \varphi}-1\right)}+F_{c}
$$


Where:

- $C_{m} \quad$ is the motor torque

- $R_{m} \quad$ is the motor pulley radius

- $\varphi \quad$ is the wrapping angle on the motor pulley

- $F_{c} \quad$ is the belt tension due to the centrifugal force.

In the equation (2.4) the wrapping angle is computed with the equation (2.5) and the centrifugal force is given by the equation (2.6).

$$
\begin{aligned}
& \varphi=180-2 \sin ^{-1}\left(\frac{R_{c}-R_{m}}{a}\right) \\
& F_{c}=\lambda \omega_{m}{ }^{2} R_{m}^{2}
\end{aligned}
$$

Where:

$-\lambda \quad$ is the mass of the belt for unit length

- $\omega_{m} \quad$ is the angular velocity of the motor pulley

- $R_{c} \quad$ is the driven pitch radius

- $R_{m} \quad$ is the motor pitch radius

- $a \quad$ is the center distance of the pulleys.

The side pressure is more difficult to compute, so, to make easier the proportioning process, the belt manufacturer advices to compute the "LT factor" with the equation (2.7).

$$
\mathrm{LT}=\frac{\mathrm{C}_{\mathrm{m}}}{\mathrm{R}_{\mathrm{m}} A_{c}}
$$

The LT factor is considered proportional to the mean side pressure, and is a function of the projection of the belt side on the motion plane of the pulley, shown as $A_{c}$ in the equation (2.7).

This kind of proportioning method cannot describe the durability of the belt, since it guides the belt selection without any information on the life time expected with a given stress level. An experimental analysis conducted by the belt producer (Mitsuboshi Belting Ltd.) has shown a strong correlation between the side contact pressure and the life time; this correlation is reported in Figure 2.3. 


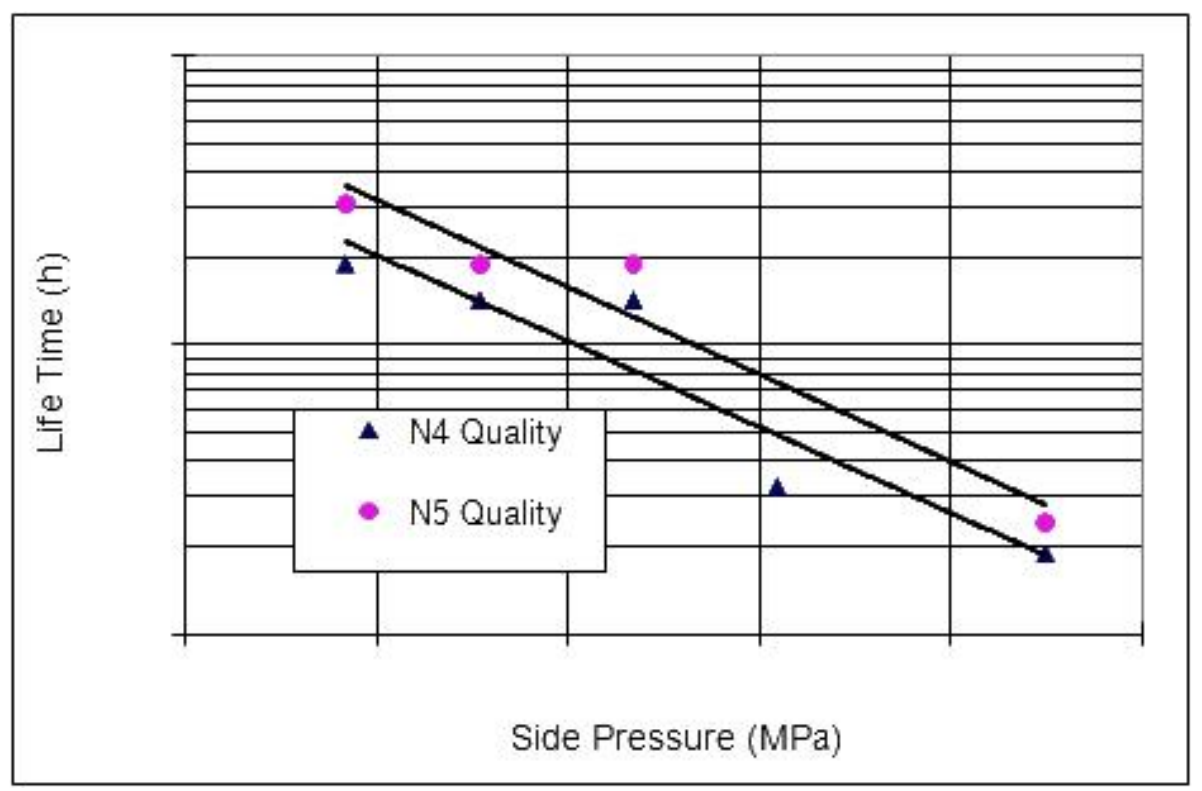

Figure 2.3: Belt life vs. side pressure

In this work we have chosen to adopt this model to compute the life time expectation of the belt, considering the load history given by the actual side pressure generated by the contact between the belt ant the pulley. To measure the side pressure acting on the belt we have realized a MultiBody model of the CVT mechanism, that will be explained in chapter 2 . In the following section we have reported the Finite Element analysis performed on the belt that has been used as an input in the MultiBody model. 


\subsection{The Finite Element modeling of the belt}

To model the CVT mechanism we have schematized the belt as a series of rigid elements connected by viscoelastic joints. The elastic behavior of these joints have been defined using a Finite Element (FE) model of a longitudinal element of the belt: imposing a known deformation in the various directions and measuring the relative reaction force, we have computed the stiffness matrix of the belt.

Assuming that the motion of the belt evolves on a plane, the joint between two belt elements can be seen as a beam element, with the stiffness matrix reported in (2.8).

$$
[K]=\left[\begin{array}{cccccc}
\frac{E A}{L} & 0 & 0 & -\frac{E A}{L} & 0 & 0 \\
0 & 12 \frac{E J}{L^{3}} & 6 \frac{E J}{L^{2}} & 0 & -12 \frac{E J}{L^{3}} & 6 \frac{E J}{L^{2}} \\
0 & 6 \frac{E J}{L^{2}} & 4 \frac{E J}{L} & 0 & -6 \frac{E J}{L^{2}} & 2 \frac{E J}{L} \\
-\frac{E A}{L} & 0 & 0 & \frac{E A}{L} & 0 & 0 \\
0 & -12 \frac{E J}{L^{3}} & -6 \frac{E J}{L^{2}} & 0 & 12 \frac{E J}{L^{3}} & -6 \frac{E J}{L^{2}} \\
0 & 6 \frac{E J}{L^{2}} & 2 \frac{E J}{L} & 0 & -6 \frac{E J}{L^{2}} & 4 \frac{E J}{L}
\end{array}\right]
$$

Where:

- $E \quad$ is the Young modulus of the material,

- $A$ is the cross section area,

- $J$ is the moment of inertia of the cross section

- $L \quad$ is the length of the beam element.

The matrix in (2.8) is completely defined once $E, A, J$ and $L$ are known. In the following sections we have reported the method used to compute the correct values to use in MB analysis. To simplify the procedure, the quantities evaluated are $L$ and the products $E A$ and $E J$.

The motion of the belt is not rigorously planar, because the variation of the transmission ratio imposes a distance between the mid planes of the two pulleys. However, this distance is small compared to the center distance between the pulleys, so the misalignment can be omitted. 
In Figure 2.4 the dimensions of the belt used in our analysis are shown.
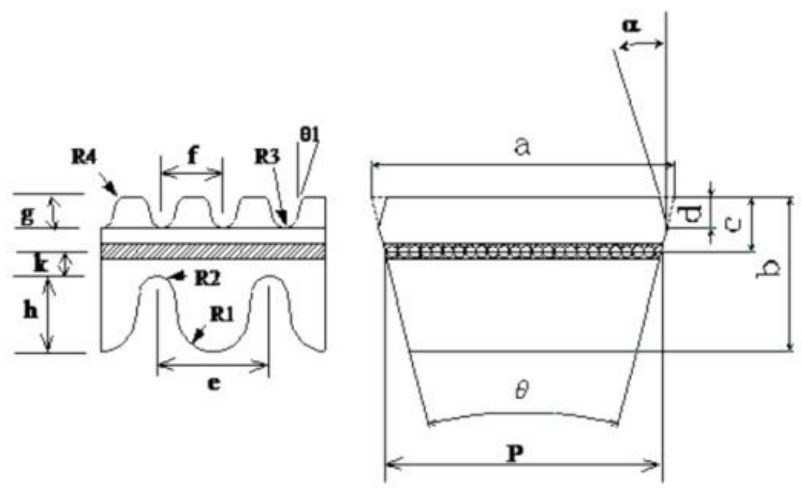

\begin{tabular}{|l|r|l|}
\hline a & 27.7 & $\mathrm{~mm}$ \\
\hline b & 14 & $\mathrm{~mm}$ \\
\hline c & 4.9 & $\mathrm{~mm}$ \\
\hline d & 2.8 & $\mathrm{~mm}$ \\
\hline e & 10.3 & $\mathrm{~mm}$ \\
\hline f & 5.6 & $\mathrm{~mm}$ \\
\hline g & 2.8 & $\mathrm{~mm}$ \\
\hline h & 6.8 & $\mathrm{~mm}$ \\
\hline k & 2.3 & $\mathrm{~mm}$ \\
\hline P & 25.3 & $\mathrm{~mm}$ \\
\hline R1 & 3.5 & $\mathrm{~mm}$ \\
\hline R2 & 1.6 & $\mathrm{~mm}$ \\
\hline R3 & 1 & $\mathrm{~mm}$ \\
\hline R4 & 0.5 & $\mathrm{~mm}$ \\
\hline$\theta$ & 28 & Degrees \\
\hline$\theta 1$ & 15 & Degrees \\
\hline$\alpha$ & 14 & Degrees \\
\hline
\end{tabular}

Figure 2.4: The belt layout

Using these data, we have realized the CAD model of the belt element, shown in Figure 2.5: the belt has been modeled dividing each component of the structure, avoiding the use of approximations used in other works [7], in order to compute the tension in each element of the belt and facilitate the evaluation of the damage accumulation for future anlyses.

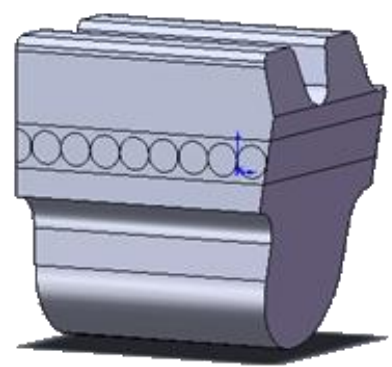

Figure 2.5: CAD model of a belt element

The elastic characteristics of each component of the belt have been provided by the supplier and the mesh has been executed using 8-nodes brick elements. 


\subsubsection{The product $E A$}

To measure the product between the equivalent cross section area and the Young modulus, we have executed a traction test on the belt element model. As shown in Figure 2.6, we have imposed to the belt element a traction force in the direction of the plies and measured its global deformation.

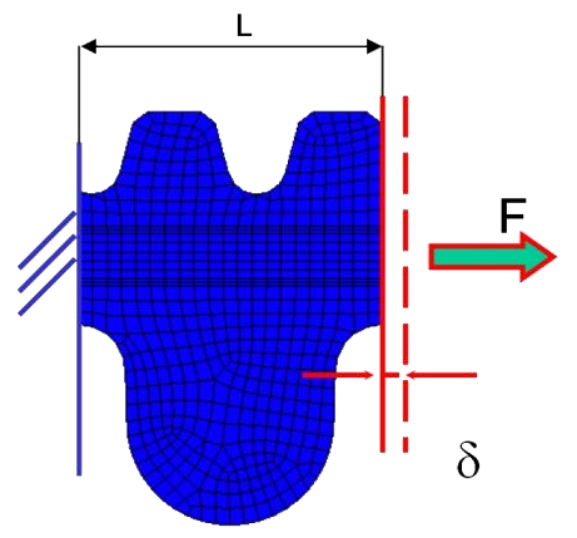

Figure 2.6: Traction test

By analogy with an homogenous, elastic isotropic material, the relation between force and deformation can be expressed as in (2.9).

$$
\delta=\frac{F L}{E_{e} A_{e}}
$$

Where:

$-\quad \delta \quad$ is the global displacement of the right section of the belt element

- $F \quad$ is the force applied to the right section of the belt element

- $\quad L \quad$ is the length of the belt element $(10.3 \mathrm{~mm})$

- $E_{e} \quad$ is the equivalent Young modulus of the belt element

- $A_{e} \quad$ is the equivalent cross section area of the belt element.

Using the equation (2.9) it is possible to compute the requested quantity. The FE analysis has given the results shown in Figure 2.7. 


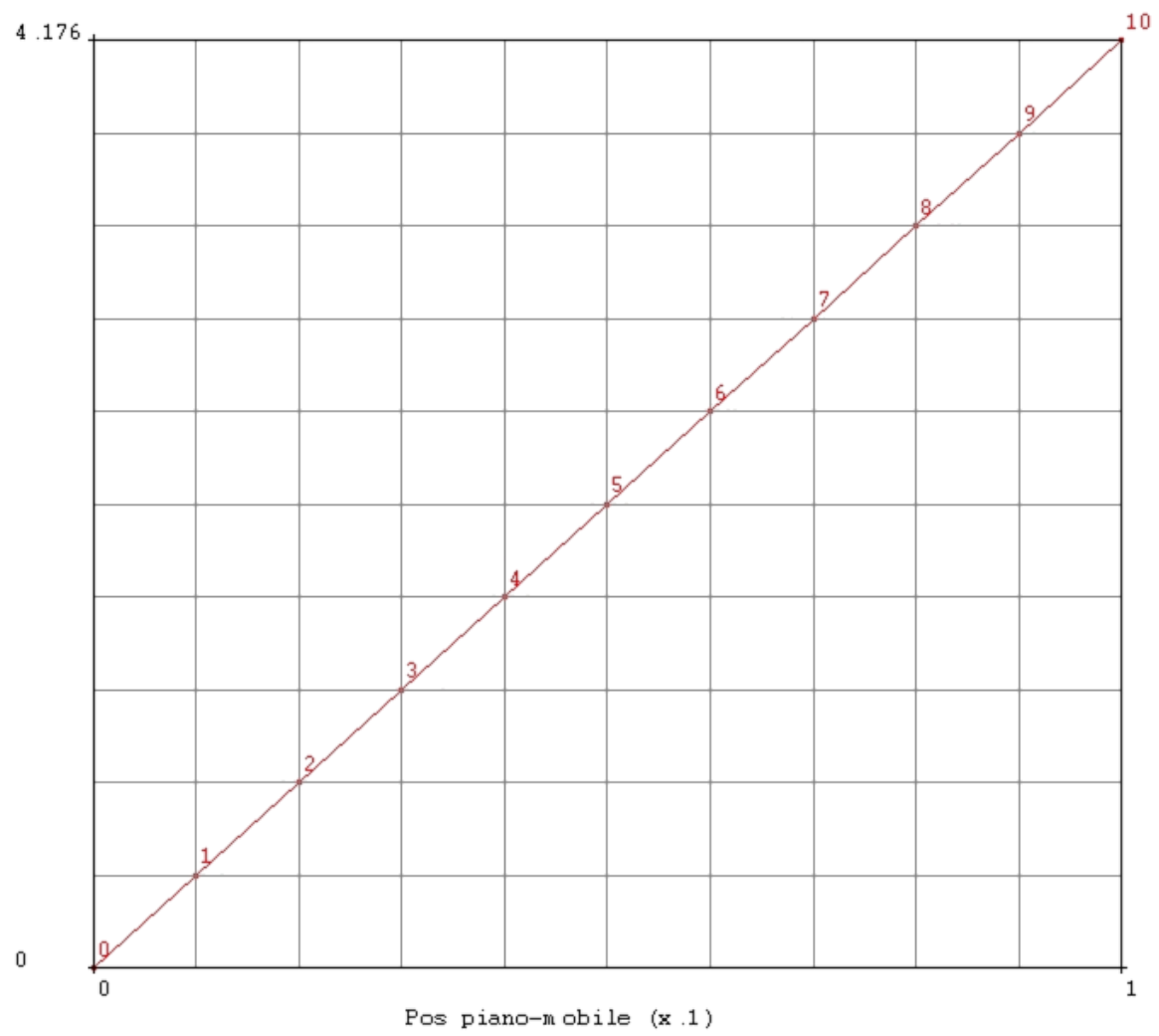

Figure 2.7: The force-displacement diagram obtained with the traction test

Remembering that the FE analysis has been performed with a symmetric model, the linear relation between force and displacement can be expressed with the equation (2.10).

$$
F=8350 \cdot \delta
$$

Therefore, the product $E_{e} A_{e}$ assumes the value shown in (2.11).

$$
E_{e} A_{e}=\frac{F L}{\delta}=8350 \cdot 10.3=86 \cdot 10^{3} \mathrm{~N}
$$




\subsubsection{The product EJ}

To measure the product between the equivalent modulus of inertia and the Young modulus of the belt, we have done a bending test on the belt element model. As shown in Figure 2.8, we have imposed to the belt element a bending moment and measured global deformation of the loading plane.

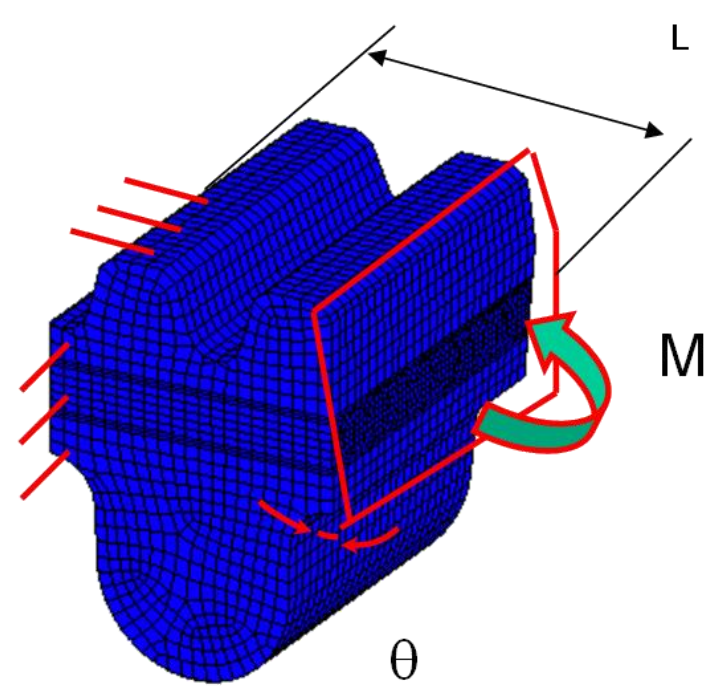

Figure 2.8: Bending test

By analogy with an homogenous, elastic isotropic material, the relation between torque and deformation can be expressed as in the equation (2.12).

$$
\vartheta=\frac{M L}{E_{e} J_{e}}
$$

Where:

- $\theta$ is the angular displacement of the right section of the belt element

- $M \quad$ is the torque applied to the right section of the belt element

- $L \quad$ is the length of the belt element

- $E_{e} \quad$ is the equivalent Young modulus of the belt element

- $J_{e} \quad$ is the equivalent moment of inertia of the belt element. 
Using the equation (6.4) it is possible to compute the requested quantity. The FEM analysis has given the results shown in Figure 2.9.

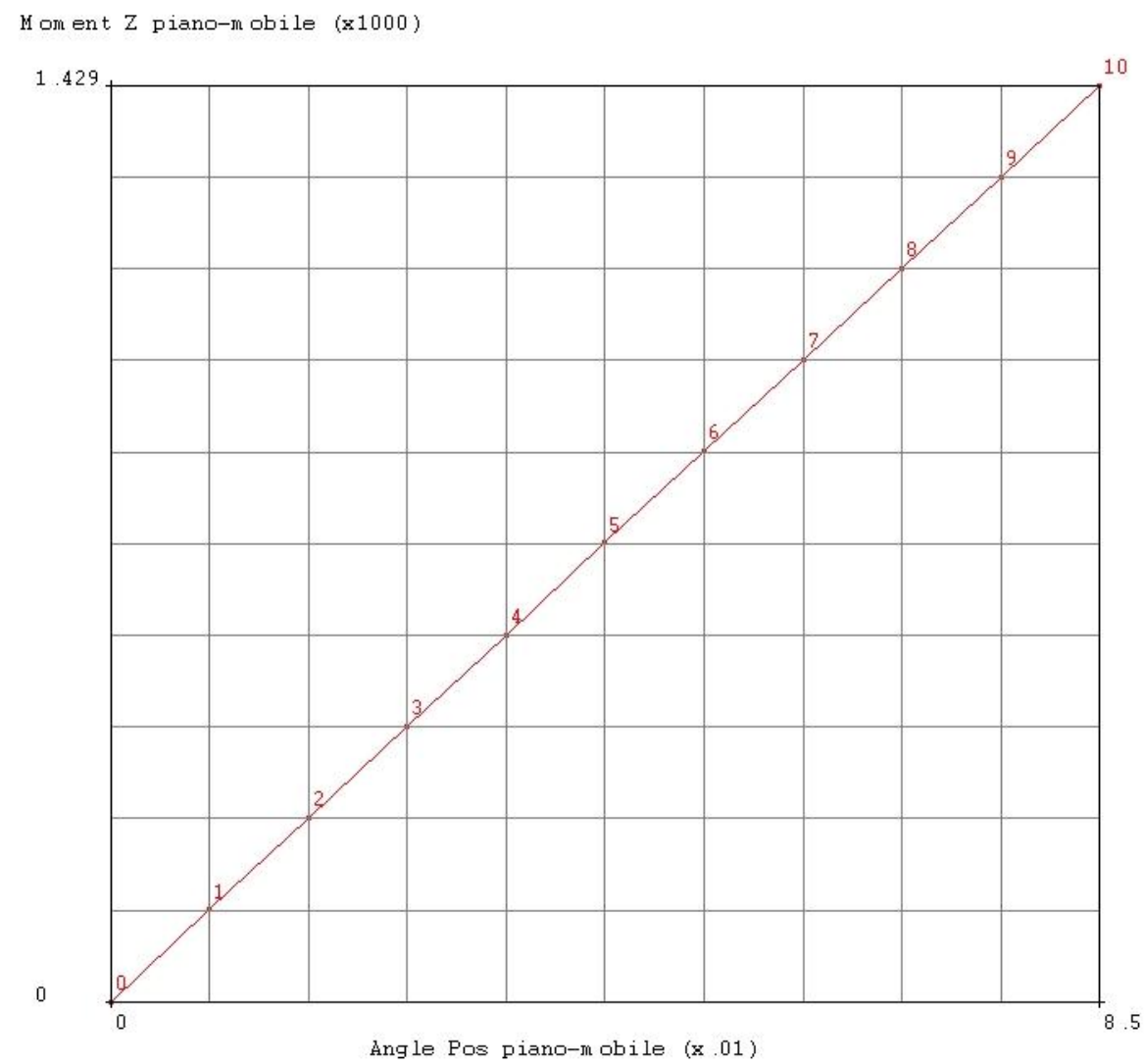

Figure 2.9: The bending moment vs. angular displacement in the bending test

Remembering that the FE analysis has been performed with a symmetric model, the linear relation between moment and angular displacement can be expressed with the equation (2.13), where $\vartheta$ is expressed in radians; therefore, the product $E_{e} J_{e}$ assumes the value shown in (2.14).

$$
\begin{aligned}
& M=2860 \cdot \vartheta \\
& E_{e} J_{e}=\frac{M L}{\vartheta}=2860 \cdot 10.3=29.5 \cdot 10^{3} \mathrm{~N} \cdot \mathrm{mm}^{2}
\end{aligned}
$$




\subsubsection{The stiffness matrix}

Using the data collected with the traction and bending tests, we can build the stiffness matrix of the belt shown in the equation (2.15).

$$
[K]=\left[\begin{array}{cccccc}
8.35 & 0 & 0 & -8.35 & 0 & 0 \\
0 & 0.324 & 1.67 & 0 & -0.324 & 1.67 \\
0 & 1.67 & 11.5 & 0 & -1.67 & 5.73 \\
-8.35 & 0 & 0 & 8.35 & 0 & 0 \\
0 & -0.324 & -1.67 & 0 & 0.324 & -1.67 \\
0 & 1.67 & 5.73 & 0 & -1.67 & 11.5
\end{array}\right] \cdot 10^{3}
$$

This formulation of the stiffness matrix has to be used with the following units:

$\begin{array}{lll}\text { - } & \text { Force unit: } & \text { Newton } \\ \text { - } & \text { Length unit: } & \text { Millimeters }\end{array}$ 


\subsection{Another possible use of the belt FE model}

The model proposed to compute the stiffness matrix of the belt could be used to formulate a new proportioning method for the belt. Considering for instance the traction test performed in the section 2.3.1, we can divide the belt structure into the following parts:

$$
\begin{aligned}
& \text { - The upper cushion } \\
& \text { - } \quad \text { The elastomeric matrix } \\
& \text { - } \quad \text { The Kevlar plies } \\
& \text { - } \quad \text { The lower cushion. }
\end{aligned}
$$

We can then define some coefficients to evaluate the stress level in the most solicited regions of the belt. For instance, in Figure 2.10 we can see the Von Mises stress field acting on the upper cushion.

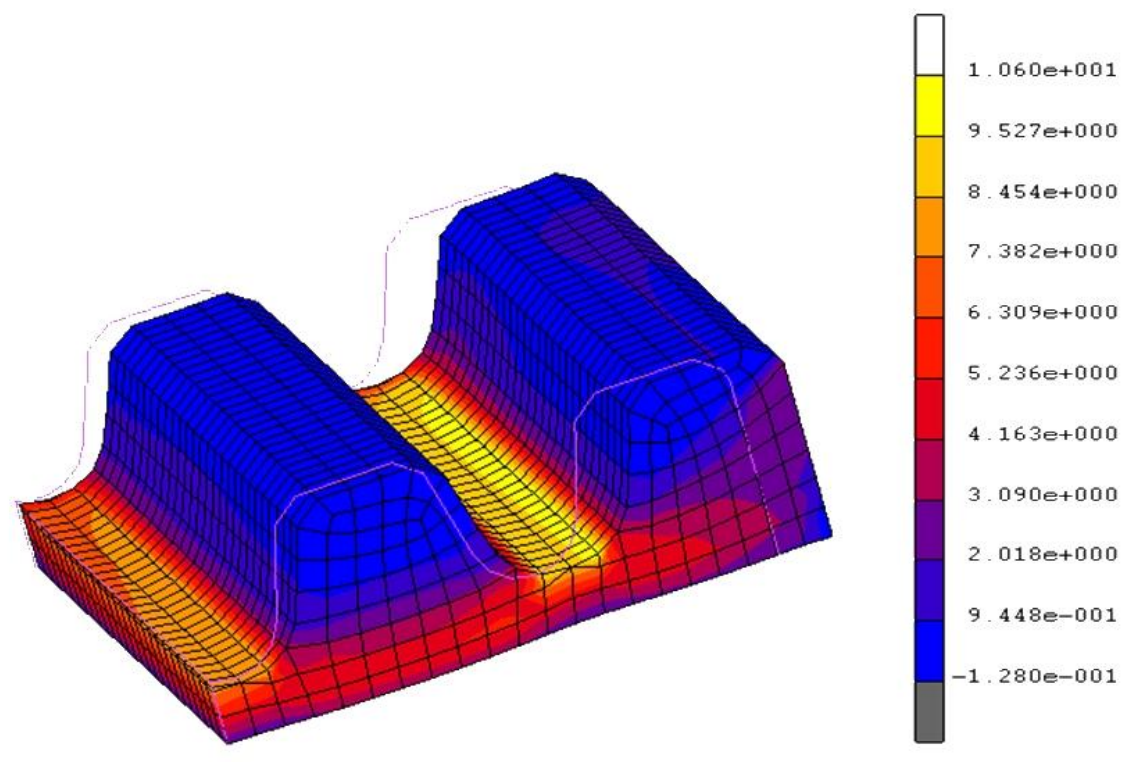

Figure 2.10: The stress field acting on the upper cushion

The most stressed region of this part is the fillet between two consecutive cogs. With the same approach, in Figure 2.11 we can see that the most stressed region in the elastomeric matrix is the one in contact with the Kevlar plies. 


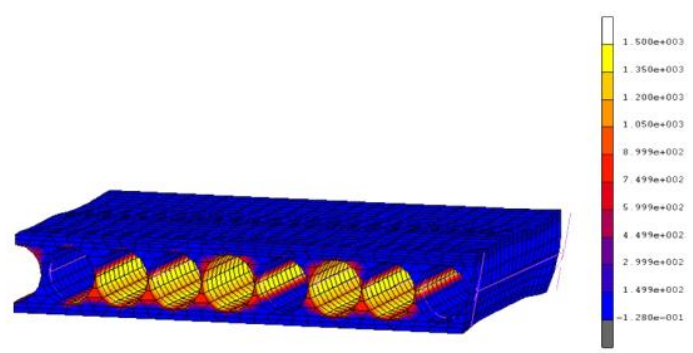

Figure 2.11: The stress field acting on the matrix

The Kevlar plies act as the most stressed part in the belt: in a traction test, their duty is to keep low the global elongation.

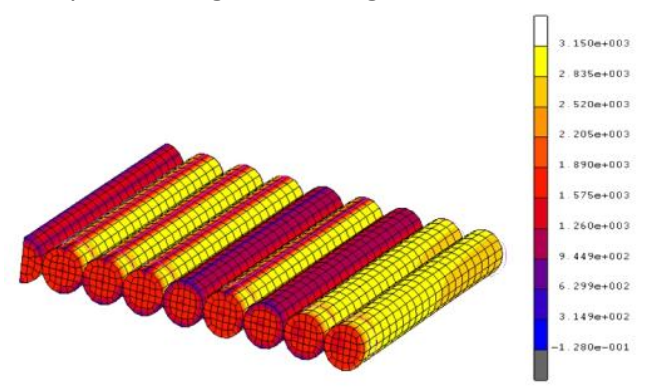

Figure 2.12: The stress field acting on the plies

The most stressed region of the lower cushion is the fillet between two consecutive teeth, as shown in Figure 2.13.

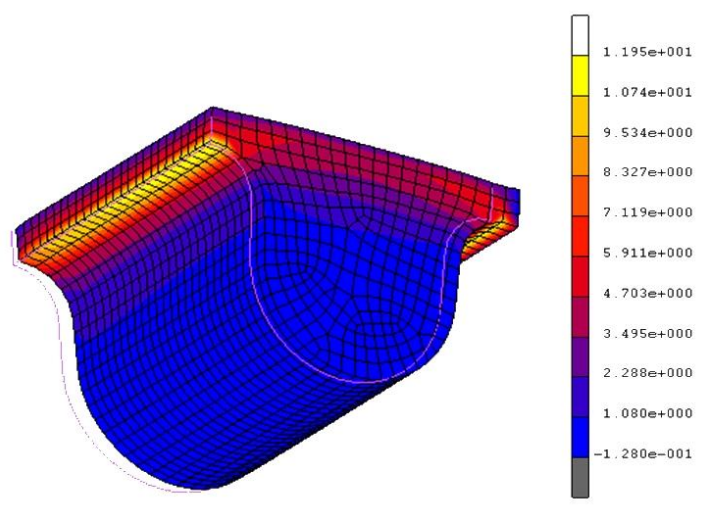

Figure 2.13: The stress field on the lower cushion 
The values assumed by the stress field in each component are referred to a generic traction force. Repeating the simulation for other values of the traction force, it is possible to define a linear correlation between the traction force and the maximum stress value assumed in each region with the equation (2.16).

$$
\lambda=\frac{\sigma_{\max }}{F}
$$

Table 2-1: Stress-Force correlation

\begin{tabular}{|c|c|}
\hline Region & $\lambda \quad\left[1 / \mathrm{mm}^{2}\right]$ \\
\hline Upper cushion & $2.53 e+4$ \\
\hline Elastomeric matrix & $3.5 e-2$ \\
\hline Kevlar plies & 7.1e-2 \\
\hline Lower cushion & $2.84 \mathrm{e}-4$ \\
\hline
\end{tabular}

The coefficient $\lambda$ shown in Table 2-1 represents the slope of the linear correlation between the traction force and the maximum stress registered in each component of the belt. These values could be used with the belt tension output of a MultiBody analysis to compute the maximum value assumed by the stress field acting on each part of the belt. These data should be used in cooperation with the belt supplier, in order to compile a new proportioning method that considers the fatigue limit of each component of the belt and evaluates its accumulated damage for the whole loading time history. 



\section{The MultiBody Analysis}

\subsection{The strategy adopted for the analysis}

The CVT mechanism is characterized by a complex interaction between the belt and the pulleys, that takes place both in the axial and in the radial direction. To keep the computational cost tolerably low, in almost all the other works in this field [6] the analyses are limited to the steady working condition. Moreover, the mechanism is always subdued to hard simplifications, like the imposition of the pitch diameters, that necessitate the calibration of some key characteristics of the model (like the belt tension) to match the experimental evidence.

In this work we will evaluate the magnitude of the forces acting on the belt and on the pulleys under steady and unsteady conditions, without any simplification of the mechanism.

To achieve this goal, we have chosen to use the software RecurDyn ${ }^{\mathrm{TM}}$, a MultiBody $(\mathrm{MB})$ software based on the recursive formulation proposed by Haug and Bae to generate the equations of motion ([8], [9], [10], [11], [12]). In most of the common $\mathrm{MB}$ analysis software, the algorithms of collision identification are related to the global Cartesian system; this approach imposes the solution of a large number of equations, since the coordinates of both bodies in contact are independent variables of the system. The software used in this work defines the coordinates in a reference system joined to one of the bodies interested by the contact (called defense body, whereas the body whose movements are expressed in the relative coordinates is called hitting body). This method allows to reduce the variable number and, as a consequence, the computational cost of the simulation ([13], [14], [15], [16]). With this method, the defense body shape is discretized with triangular shell elements and the hitting body is represented with a series of spheres.

To keep the computational cost still low, the identification of body collisions is done in two consecutive steps, with increasing computational complexity ([17], [18]). In our model, the belt - pulleys contact has been represented assuming the pulleys as the defense body and the belt element as the hitting bodies, that are equipped with five contact spheres on each side, as shown in Figure 3.1. 


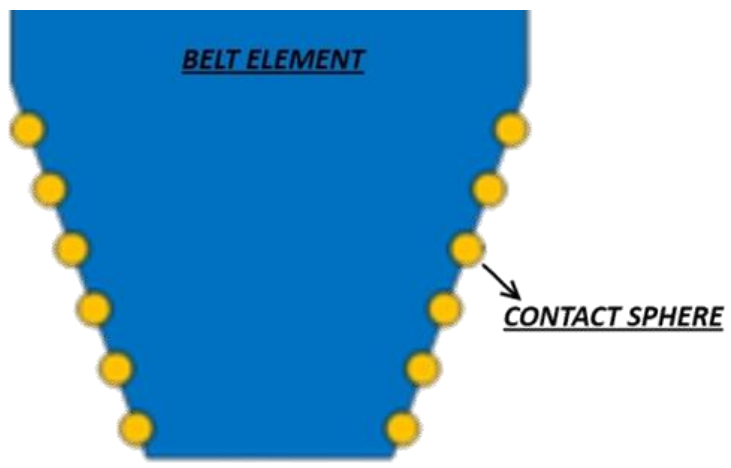

Figure 3.1: The hitting body discretization 


\subsection{An outline of the CVT Model}

To describe accurately every component of the CVT, we have modeled the system analyzing any kinematic constraint of the assembly. As shown in Figure 3.2 , we have implemented all the parts of the driveline.

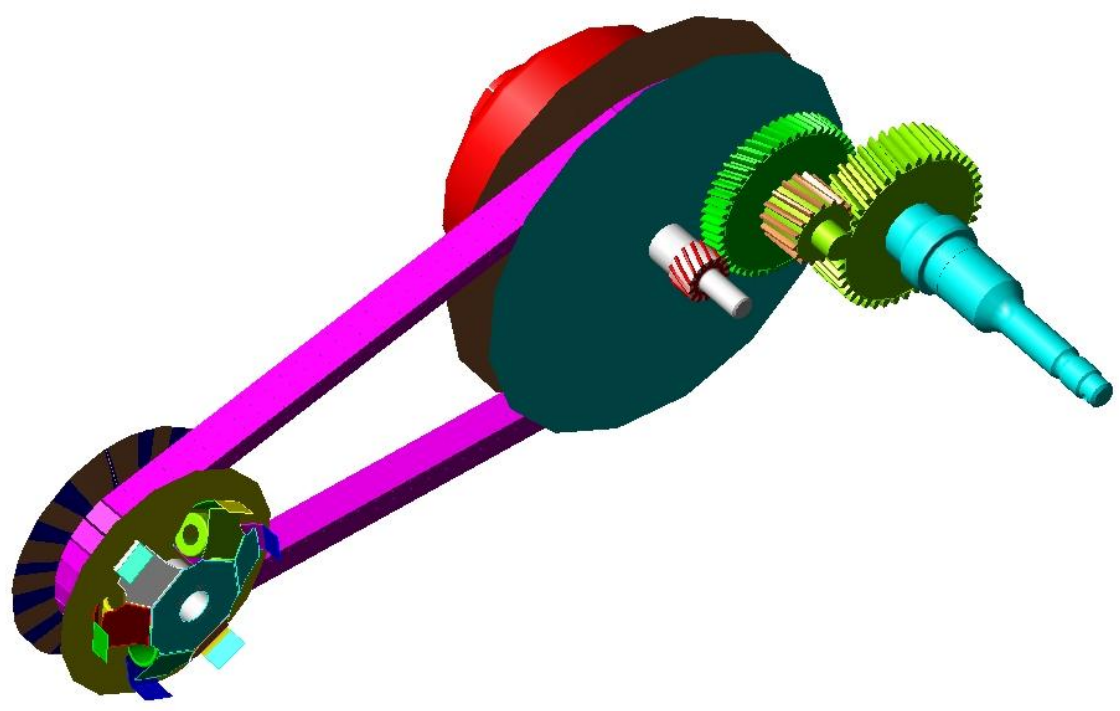

Figure 3.2: The complete MBS model of the CVT

In Figure 3.3 are shown the rollers that, moving under the effect of the centrifugal force among the grooves milled on the two half pulleys, impose the variation of the pitch diameter of the motor pulley. 


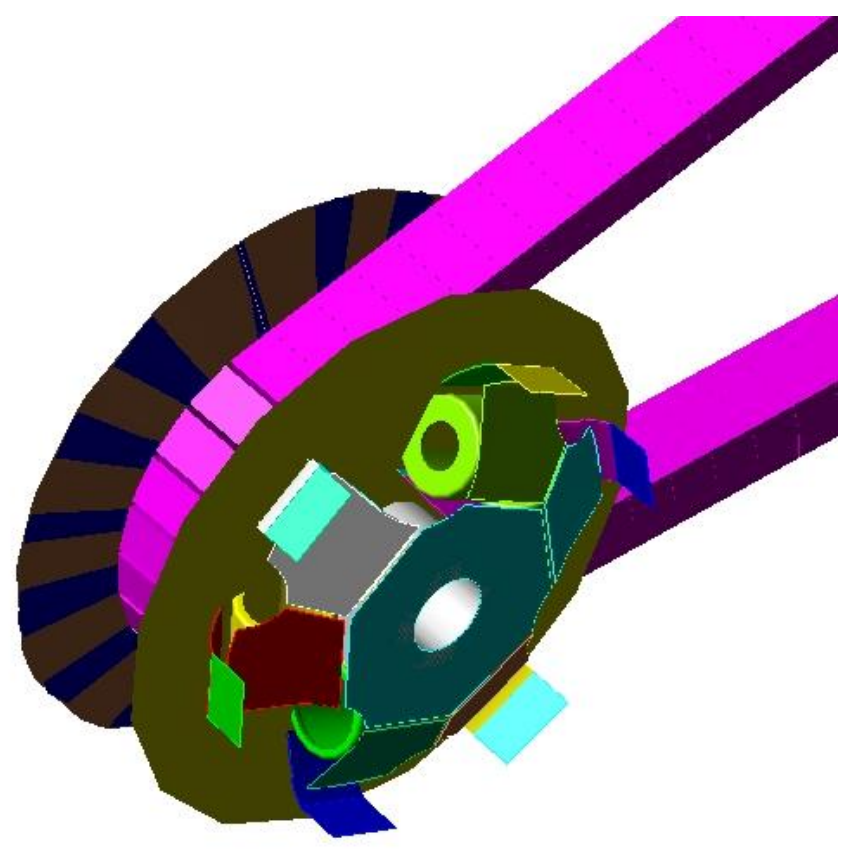

Figure 3.3: The motor pulley

Due to the high elongation stiffness of the belt, an increase of the pitch diameter of the motor pulley is followed by a reduction of the pitch diameter of the driven pulley (and vice versa). This reduction is opposed by the action of a spring joined to a sleeve coupling (called torque corrector) fixed to one half of the driven pulley. The other half of the pulley is joined with the driven shaft, which comes equipped with some pivots coupled with the slots of the torque corrector (see Figure 3.4). This system is designed to impose a non linear law to the force generated by the spring. To complete the model, we have joined to the driven pulley the clutch assembly and the speed reduction gear connected to the wheel. 


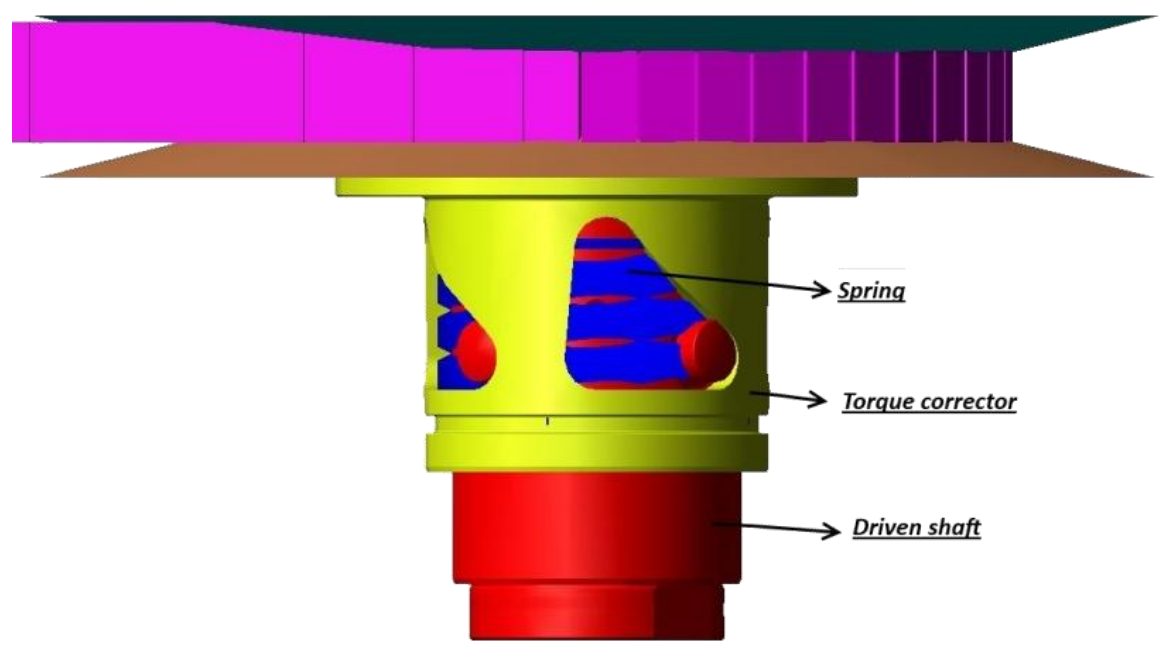

Figure 3.4: The torque corrector

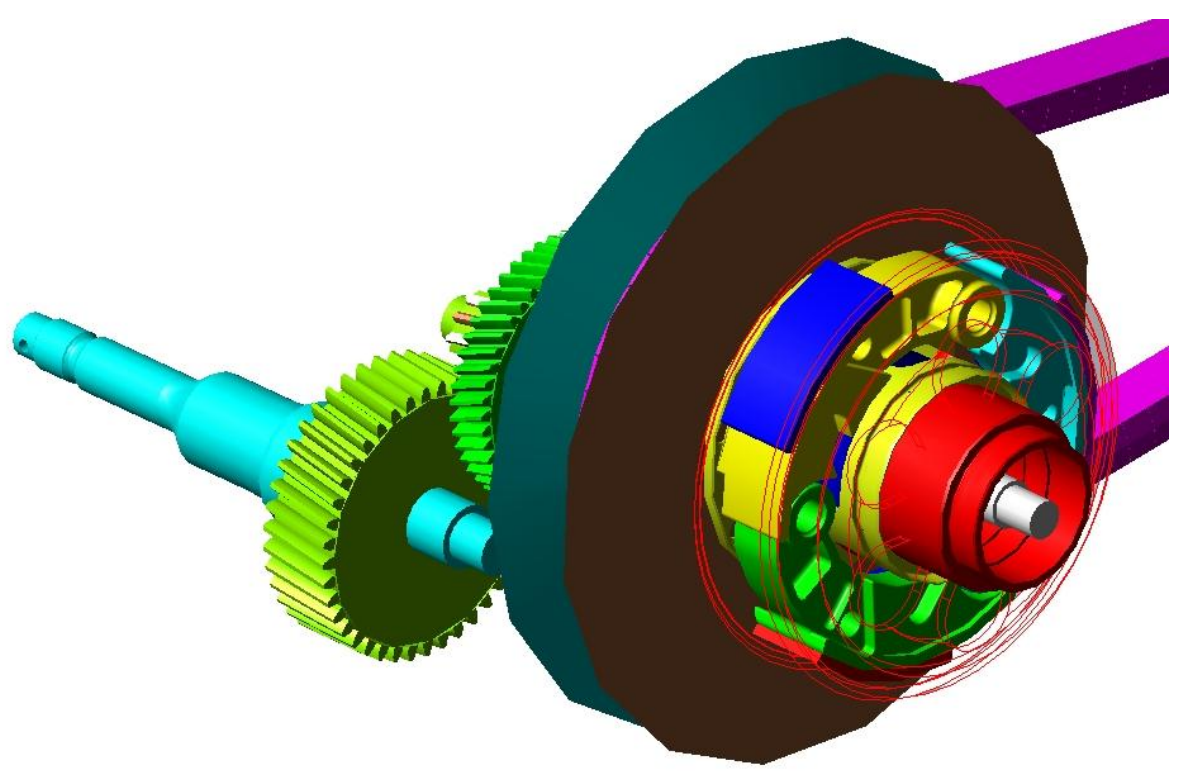

Figure 3.5: The rear assembly 


\subsection{The calibration of the model}

The contact between belt and pulley has been modeled with the pure penalty formulation, that in RecurDyn expresses the normal contact force with the equation (3.1).

$$
F_{n}=k \delta^{m_{1}}+c \frac{\dot{\delta}}{|\dot{\delta}|}|\dot{\delta}|^{m_{2}} \delta^{m_{3}}
$$

where:

- $\quad k \quad$ is the contact stiffness

- $\quad c \quad$ is the contact damping coefficient

- $\delta \quad$ is the penetration of the elements of the hitting body into the defense body

$-\delta \quad$ is the rate of penetration

- $m_{1}$ is the stiffness exponent, used to impose a non - linear behavior to the contact force

- $m_{2}$ is the damping exponent

- $m_{3}$ is the indentation exponent, used to avoid negative values of contact force.

Assuming the belt as the only deformable part of the contact pair, to evaluate the contact stiffness in the equation (3.1) we have analyzed the behavior of the FE model described in chapter 2.3 under the action of a lateral force. As shown in Figure 3.6, we have joined the belt element to the ground on its symmetry plane, and applied a known compression force with a rigid plane on the contact side.

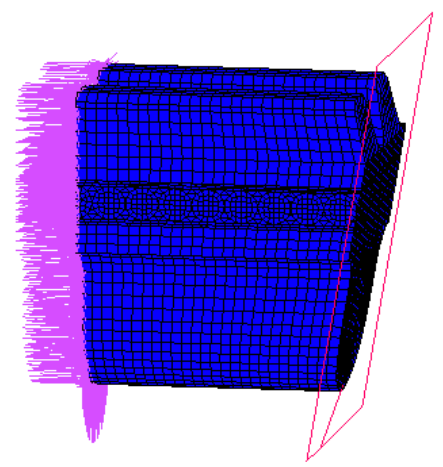

Figure 3.6: The model used to define the contact stiffness 
The displacement of the pushing plane has been used to evaluate the contact stiffness.
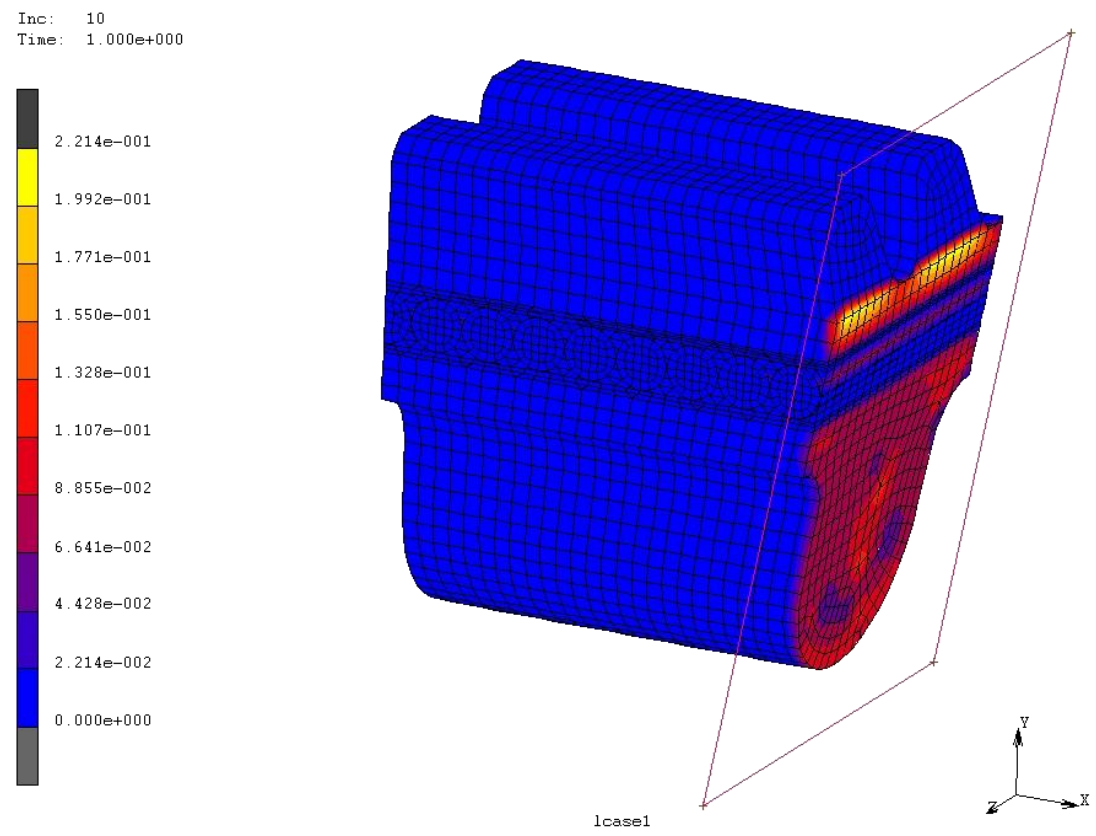

Contact Normal Force

Figure 3.7: Contact normal force distribution

In Figure 3.8 is shown the correlation between the actuation force and the displacement of the loading plane. 


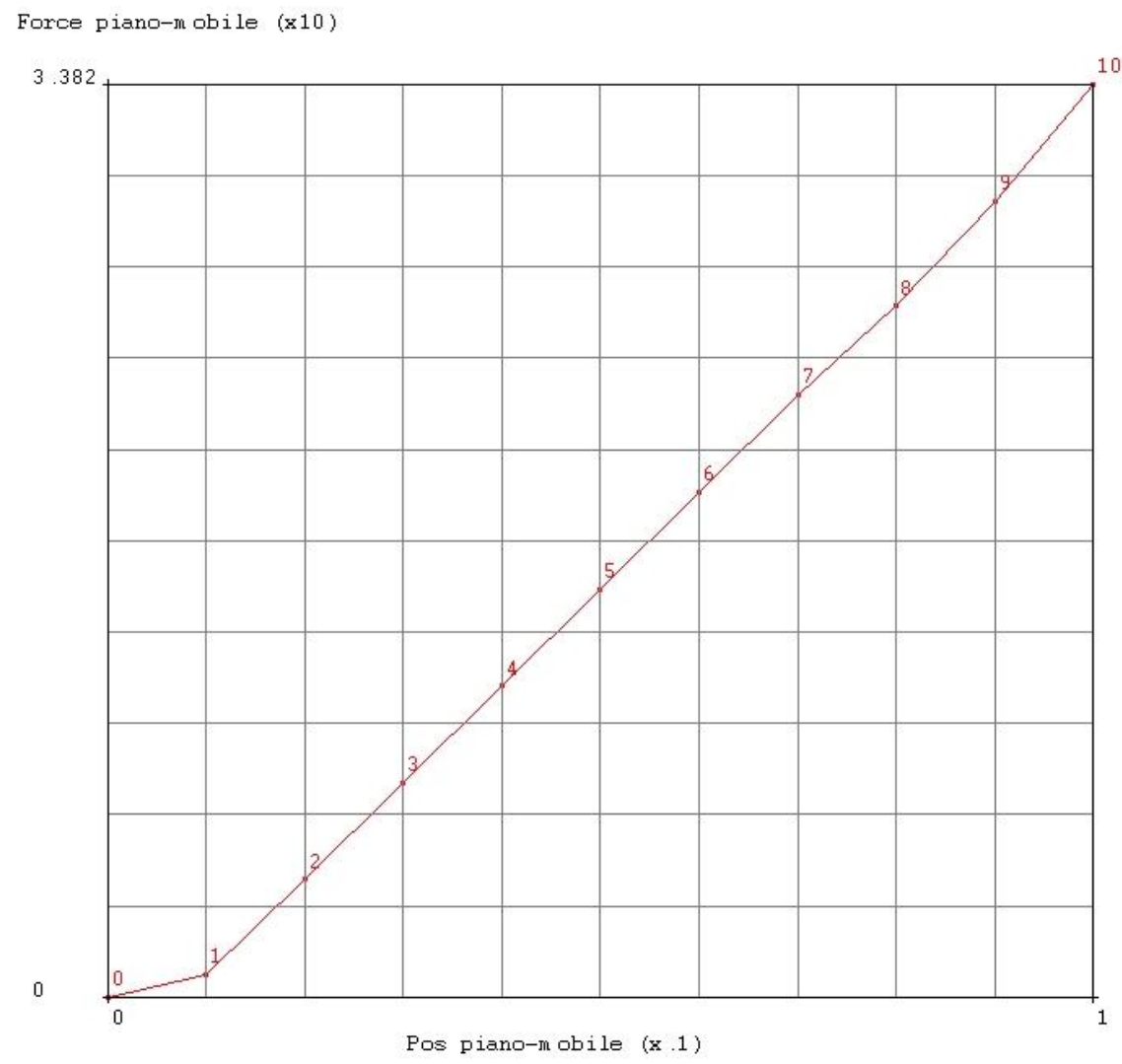

Figure 3.8: Force-Displacement behavior with a lateral force

We have used the data shown in Figure 3.8 to get the coefficients $k$ and $m_{1}$ needed to define the elastic part of the contact formulation. The values of the contact stiffness and of the stiffness exponent have been chosen in order to minimize the error in respect to the FE analysis data. The best fitting equation for the elastic term is shown in (3.2).

$$
F=600 \cdot \delta^{1.245}
$$

This equation allows to keep the error below $5.5 \%$ of the force in almost all the integration points. As shown in Figure 3.9, the error reaches a maximum for a small displacement of the loading plane. Even if the error is great in percentage, its absolute value is lower than $1.1 \mathrm{~N}$ and occurs for a force lower than the expected one in the contact between the belt and the pulley. 


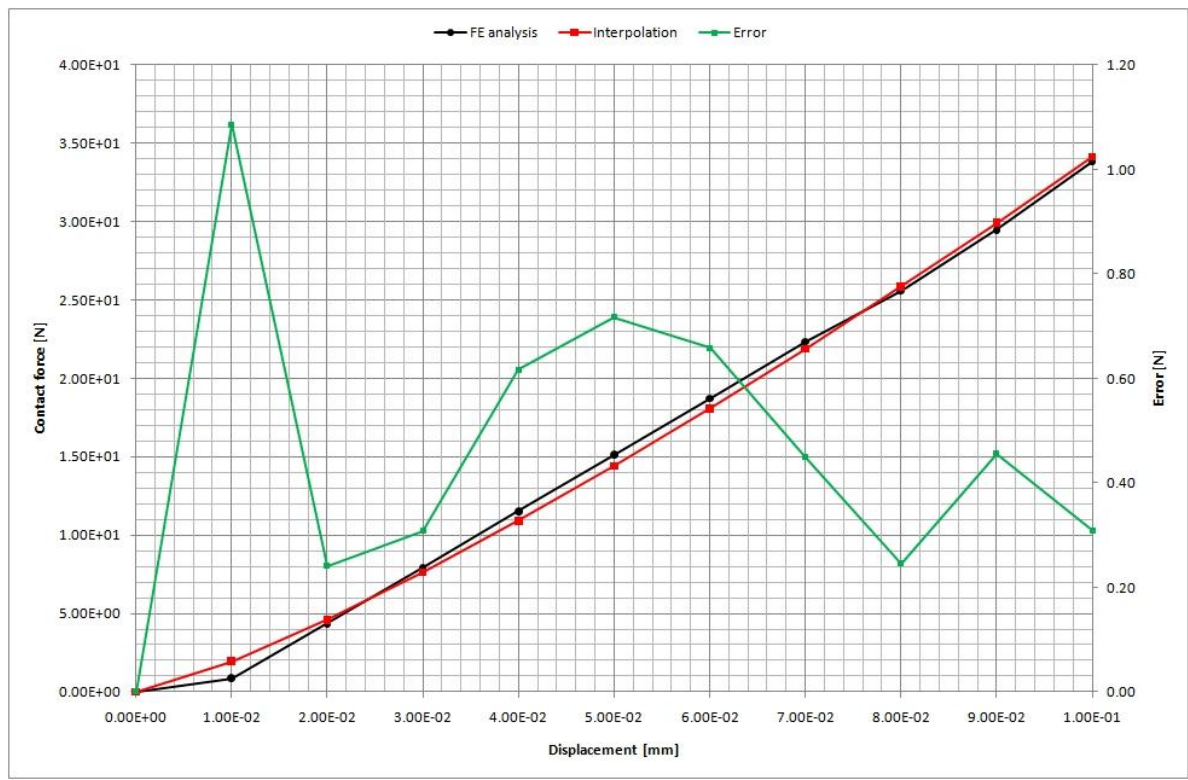

Figure 3.9: Calibration of the elastic part of the contact formulation

Unlike other works [19], the proposed MB model does not require the calibration of the preload of the belt, since the pitch diameters of both pulleys can vary in order to adapt the system to the elasticity of the belt.

The damping coefficient results to be more difficult to evaluate by numerical or experimental tests. Therefore, we have conducted the calibration of this parameter with a Design of Experiments (DOE) approach. This approach, used also to evaluate the damping matrix of the belt element, has been conducted with the aim of keeping the computational cost low, in respect of the results stability.

We have chosen to execute the DOE analysis on a simplified model of the CVT system, which omits the pitch diameter variation in both the motor and driven pulleys. The simplified CVT has the same features of the original one (in terms of geometry and inertia properties), but the half pulleys are joined together in the axial direction; this simplification has allowed a great saving in simulation time. Imposing a variation of the investigated parameters of the simulation (into a consistent range), we have monitored the corresponding variation in some key output data, like belt tension and actual transmission ratio. Discarded the parameters that lead to a non consistent behavior of the system, we have chosen the parameters that ensure a saving in simulation time. 


\subsection{Simulations and result}

We have taken advantage of the described model to produce the typical load history needed to proportionate the belt and the pulleys with a damage accumulation approach. Taking into account the standard Piaggio mission profile for the engine bench tests, called $\Delta t$ the whole life cycle, the following quantities have then been defined:

$$
\begin{array}{ll}
- & \Delta t_{t}=0.05 \Delta t \\
- & \Delta t_{p}=0.85 \Delta t \\
- & \Delta t_{o}=0.10 \Delta t \\
- & f_{t}=5750 \mathrm{rpm} \\
- & f_{p}=7700 \mathrm{rpm} \\
- & f_{o}=8500 \mathrm{rpm}
\end{array}
$$
fraction of $\Delta t$ at maximum torque fraction of $\Delta t$ at maximum power fraction of $\Delta t$ in over speed engine speed at maximum torque engine speed at maximum power engine speed in over speed.

During $\Delta t_{t}$ the engine completes $N_{t}$ revolutions, where $N_{t}$ is given by the equation (3.3).

$$
N_{t}=f_{t} \Delta t_{t}
$$

Similar considerations apply to $\Delta t_{p}$ and $\Delta t_{o}$. So the number of rotations are given by the equations (3.4) and (3.5).

$$
\begin{aligned}
& N_{p}=f_{p} \Delta t_{p} \\
& N_{o}=f_{o} \Delta t_{o}
\end{aligned}
$$

We have performed the simulation using these parameters, to define the lifetime expected for the belt with a typical load history that could be used to design the belt with the aid of the graph reported in Figure 2.3. If the belt producer supplied the material characteristics, a more detailed analysis of the damage accumulation of the belt would be performed, using the guidelines introduced in chapter 2.4. The belt fatigue data shown in Figure 2.3 are referred to a test condition, where the pulleys act a constant pressure on the belt, with the motor pulley rotating at $4200 \mathrm{rpm}$. In these conditions, the loaded part of the belt (i.e. the part of the belt constrained in the pulleys) is $50 \%$ of its total length $(665 \mathrm{~mm})$, and the linear velocity of the belt is $11.7 \mathrm{~m} / \mathrm{s}$. From these data we can notice that one hour of test coincides with approximately 127000 load cycles. We will use this value to define the lifetime of the belt.

We have performed all the simulations starting from a static configuration, in order to evaluate the stress gain connected to the acceleration of the driven wheel. 


\subsubsection{Maximum torque conditions}

This analysis has been conducted imposing a constant motor torque equal to $76.4 \mathrm{Nm}$ at $5750 \mathrm{rpm}$. We have also imposed an opposing toque on the driven pulley equal to $176 \mathrm{Nm}$, as prescribed by Piaggio. In Figure 3.10 we can notice the difference in belt tension between the tight side and the slack one, that balances in the drive pulley the motor torque.

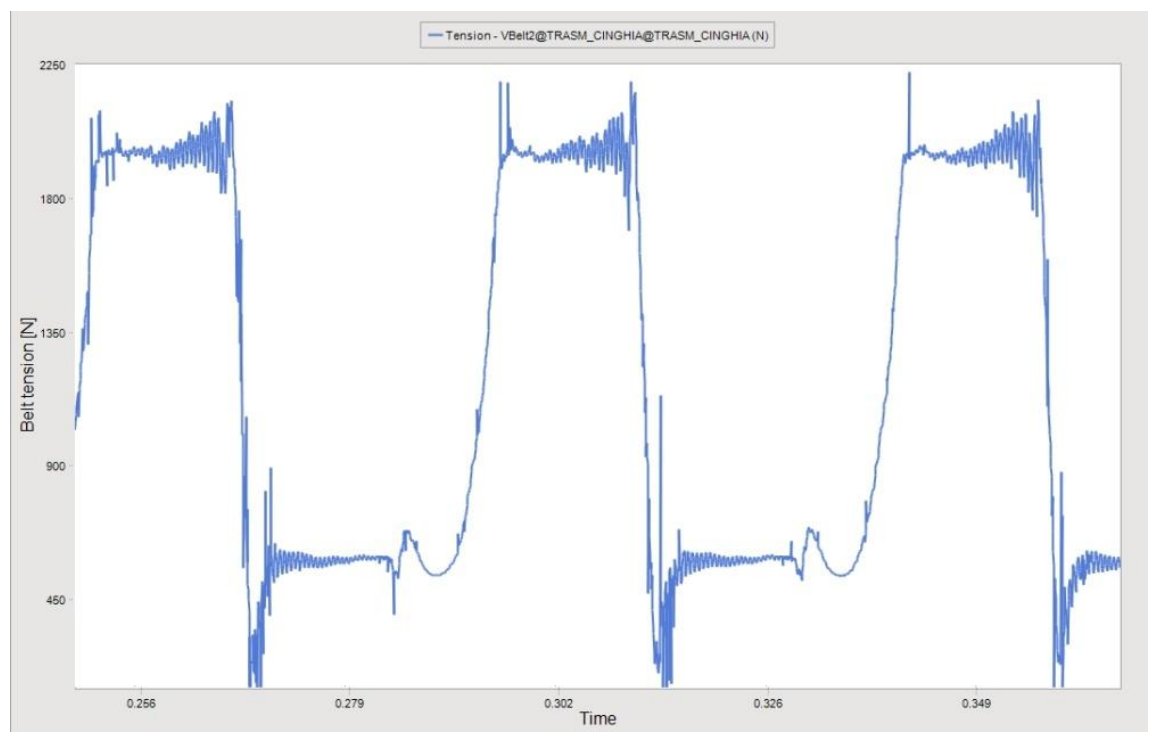

Figure 3.10: Belt tension in the maximum torque test

In Table 3-1 we have reported the characteristics of the belt tension history relative to this load case.

Table 3-1: Belt tension history in maximum torque

\begin{tabular}{|cccc|}
\hline Tight side & Slack side & Mean tension & Alternate tension \\
\hline $2050 \mathrm{~N}$ & $470 \mathrm{~N}$ & $1260 \mathrm{~N}$ & $790 \mathrm{~N}$ \\
\hline
\end{tabular}

The alternate and the mean tension could be used in a Wöhler-like diagram of the belt to define the damage level connected with this load case. Since these data are missing, by now we can only use the maximum value reached by the belt tension to select the most appropriate belt for our application.

In contrast with the common proportioning systems, the Figure 3.11 shows that the contact force is not constant along the wrapping angle, but shows some spikes due to the elastic behavior of the belt. 


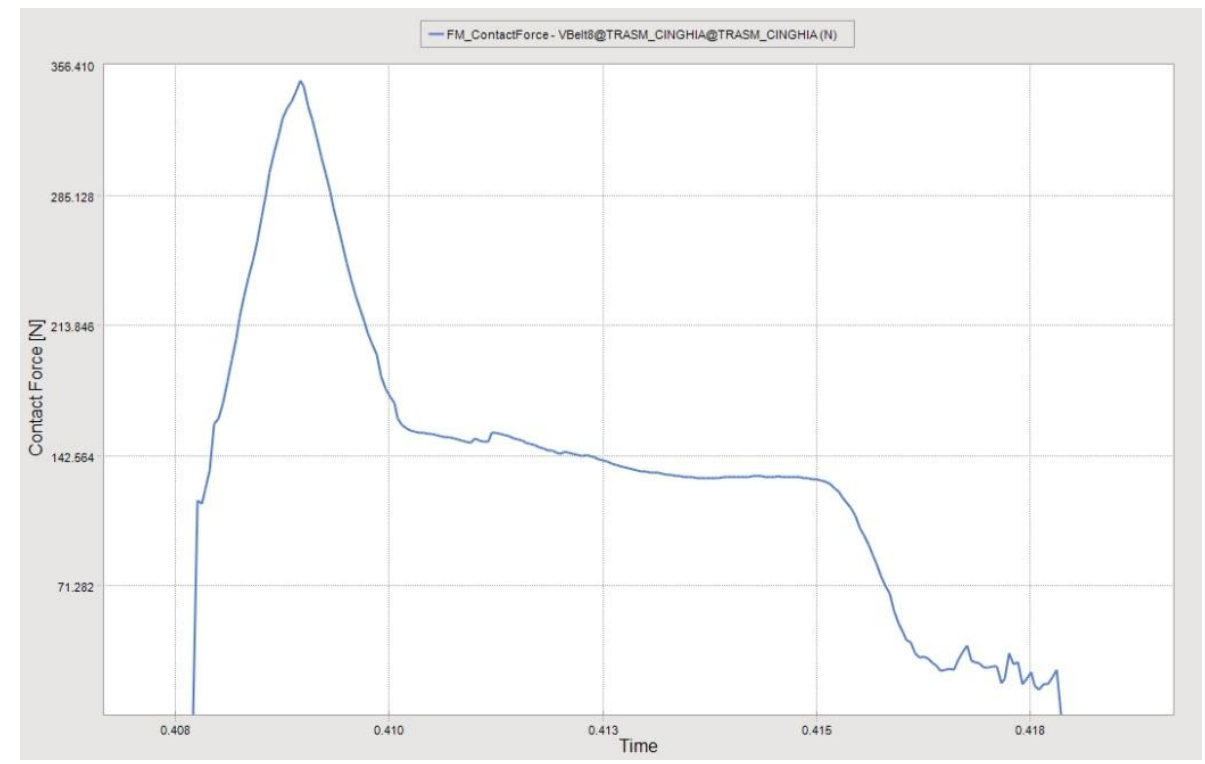

Figure 3.11: Contact force on the motor pulley in maximum torque conditions

The contact forces reach their maximum value entering the drive pulley by reason of the compression work carried on the belt. As shown in Figure 3.12, the first segment of the pulley must withstand the elastic reaction generated by the free side of the belt, where the wedging entails the compression of the belt.

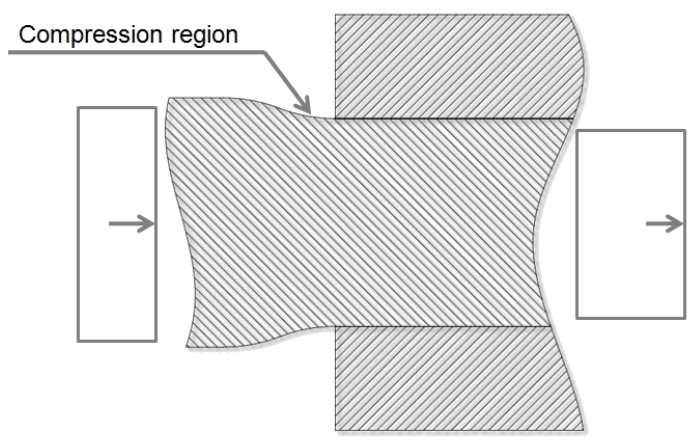

Figure 3.12: The compression work done by the pulleys to the belt

The same behavior can be noticed in the driven pulley, as shown in Figure 3.13 , where we can see a second peak in the force diagram, that takes place 
in the slipping arc: in the same figure we can notice that the contact force begins to rise when the belt element starts assuming the rectilinear motion. This behavior finds confirmations also in experimental results available in literature [20].

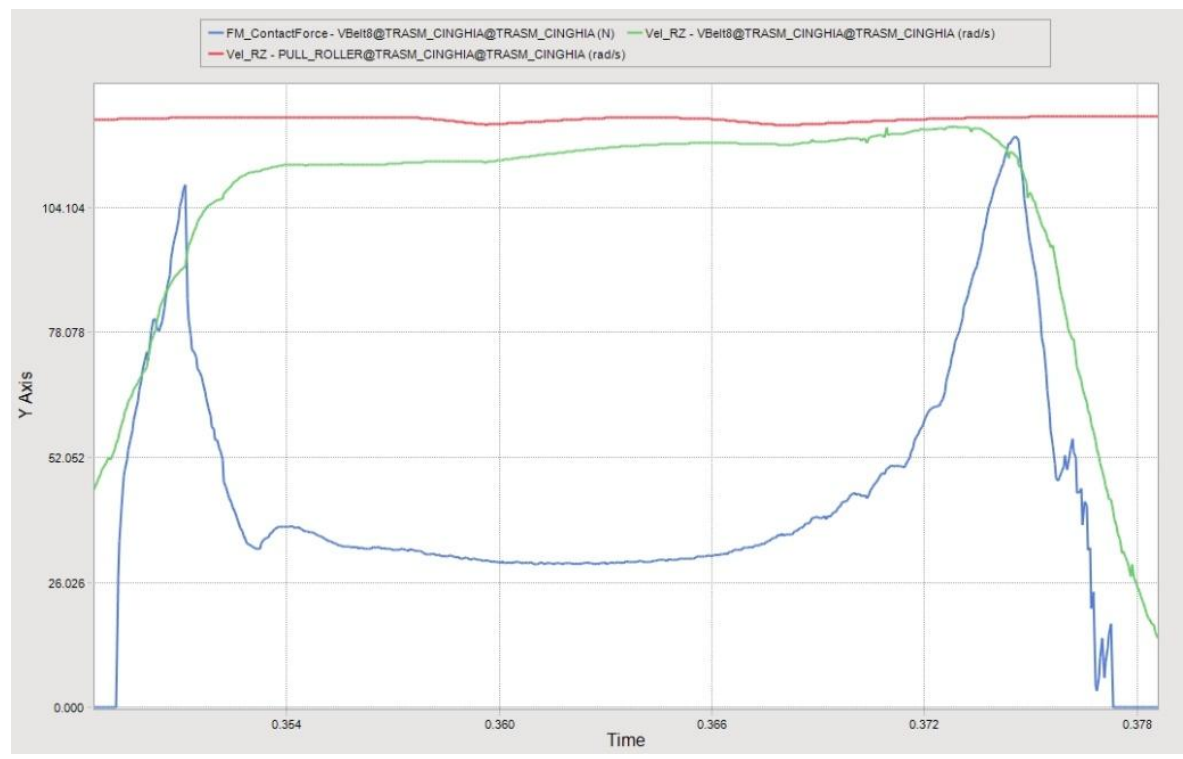

Figure 3.13: Contact force on the driven pulley (blue line), angular velocity of the pulley (red line) and of the belt element (green line) in the maximum torque test

In the Table 3-2 are reported the data useful to interact with the fatigue diagram shown in Figure 2.3. The side contact pressure values are computed considering the area of the lateral surface of the belt elements $\left(82 \mathrm{~mm}^{2}\right)$.

Table 3-2: Data for belt durability in maximum torque

\begin{tabular}{|l|r|}
\hline Mean pressure in the driven pulley & $0.6 \mathrm{MPa}$ \\
\hline Mean pressure in the motor pulley & $1.7 \mathrm{MPa}$ \\
\hline Belt total length & $970 \mathrm{~mm}$ \\
\hline Angular velocity of the driven pulley & $270 \mathrm{rad} / \mathrm{s}$ \\
\hline Linear velocity of the belt & $21.2 \mathrm{~m} / \mathrm{s}$ \\
\hline
\end{tabular}


Adopting a damage accumulation approach, we can compute the life time of the belt considering two separate load cases: one for the side contact pressure due to the passage of the belt into the groove of the driven pulley and one for the side pressure related to the motor pulley. In analogy with the test setup, we have chosen to use in this analysis only the mean values measured in each pulley.

Given the belt length and its linear velocity, we can compute that a belt element completes $114000\left(N_{t}\right)$ revolutions per hour. Therefore we can affirm that a belt element is subdued to each load cycle $N_{t}$ times per hour. With the best fitting curve of the graph shown in Figure 2.3 we can compute the critical life times:

$$
\begin{aligned}
& \text { - } L_{d}=460 \mathrm{~h} \text { for the load case related to the driven pulley } \\
& \text { - } L_{m}=10 \mathrm{~h} \text { for the load case related to the motor pulley. }
\end{aligned}
$$

As stated in the chapter 3.4, these durations are referred to the test bench setup. To obtain a more general indication, we have to compute the number of load cycles that bring to failure in the testing conditions with the product (3.6).

$$
N_{i}=127000 \cdot L_{i}
$$

Therefore, the critical number of cycles for each load case are:

- $\quad N_{d}=5.80 E+07$ for the load case related to the driven pulley

- $\quad N_{m}=1.30 E+06$ for the load case related to the motor pulley.

Considering this working condition, we can compute the damage $\left(D_{\mathrm{t}}\right)$ accumulated by the belt in one hour, with the Miner approach.

$$
D_{t}=\frac{N_{t}}{N_{d}}+\frac{N_{t}}{N_{m}}=0.095
$$

Considering then the first phase of the simulation, we can highlight the behavior of the transmission under unsteady conditions. In Figure 3.14 we can notice that the belt tension of the tight side reaches a maximum in the first phase of the simulation. The belt tension raise can be quantified in $40 \%$ of the value measured in the steady condition (2050N in place of $1470 \mathrm{~N}$ ). As shown in Figure 3.15 even the contact force results amplified in the unsteady conditions, raising by $35 \%$ of the value measured in steady conditions ( $490 \mathrm{~N}$ in place of $360 \mathrm{~N}$ ). 


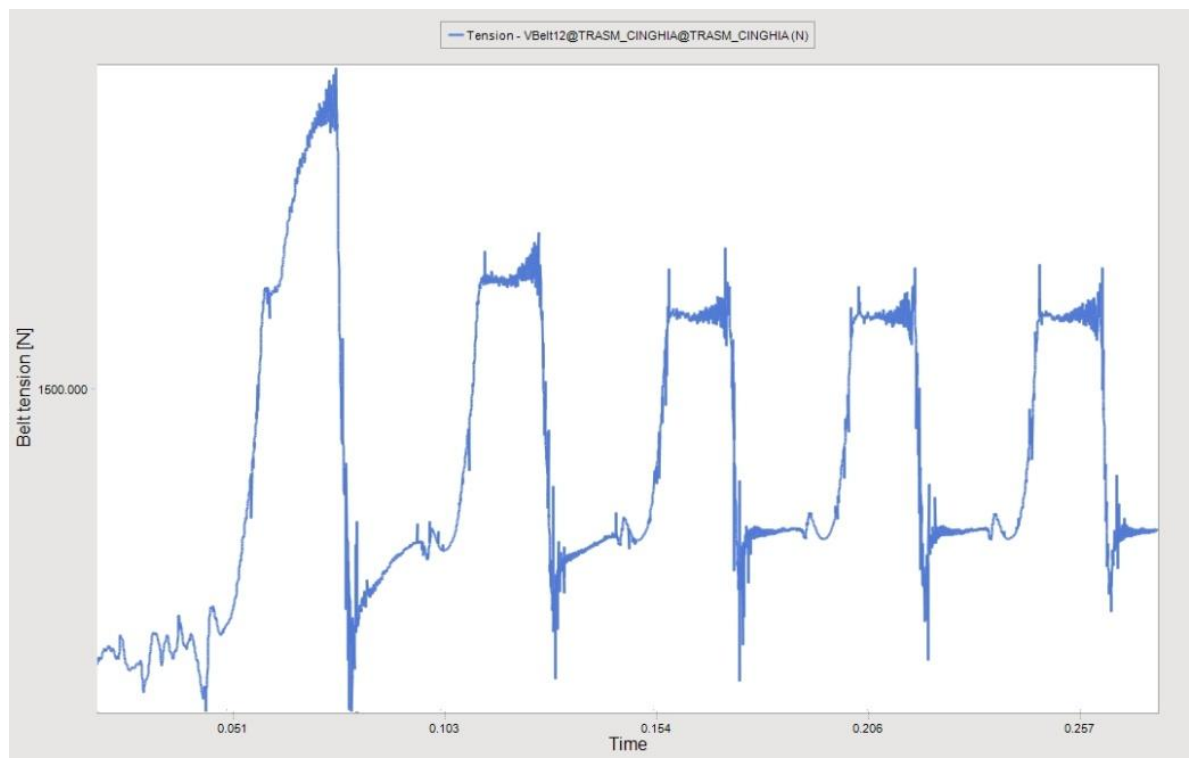

Figure 3.14: Belt tension in unsteady conditions (maximum torque)

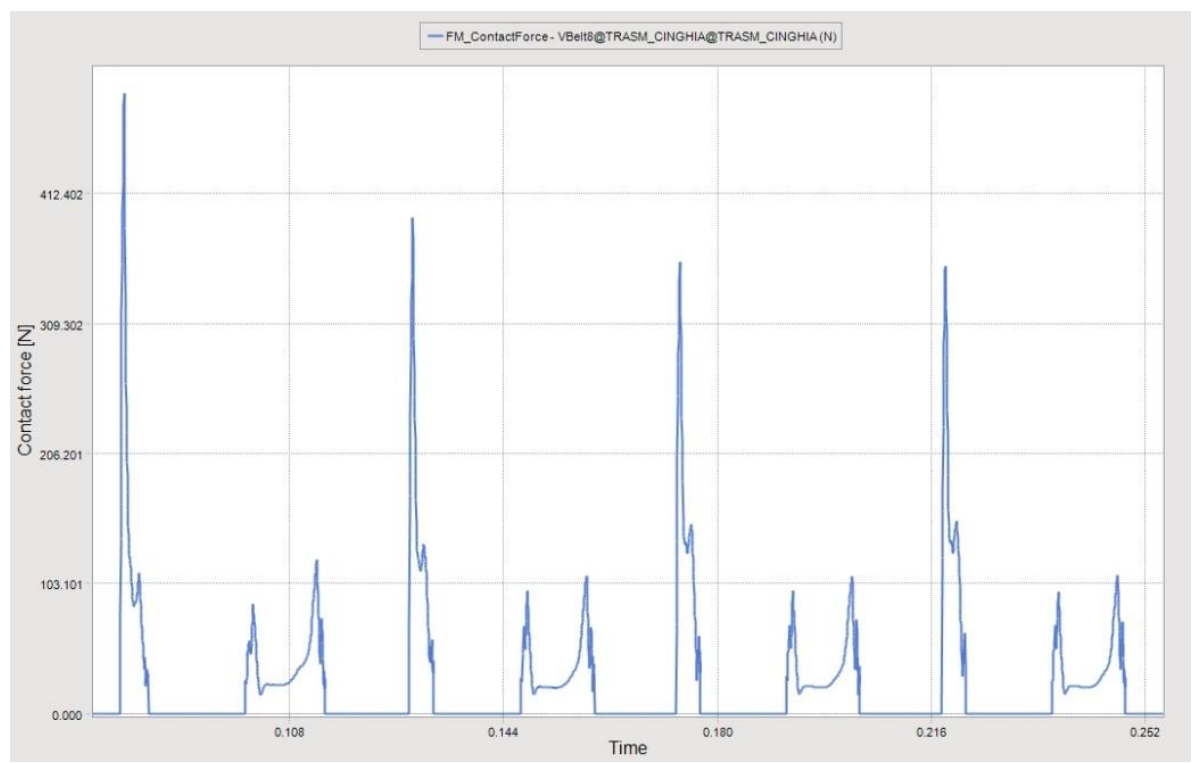

Figure 3.15: Side contact force in unsteady conditions (maximum torque) 
This behavior is mainly due to the actions of the opposing spring and of the torque corrector, that impose a delay to the opening of the driven pulley: since these two components work in opposition with the action of the rollers (see chapter 3.2), the belt is subdued to an additional traction force, that imposes the measured raise of belt tension and contact force. 


\subsubsection{Maximum power conditions}

This analysis has been conducted imposing a constant motor torque equal to the value of $68 \mathrm{Nm}$ at $7700 \mathrm{rpm}$. We have also imposed an opposing toque on the driven pulley equal to $58.5 \mathrm{Nm}$, as prescribed by Piaggio. As shown in Figure 3.16 , the belt tension load history relative to this load case can be schematized in Table 3-3.

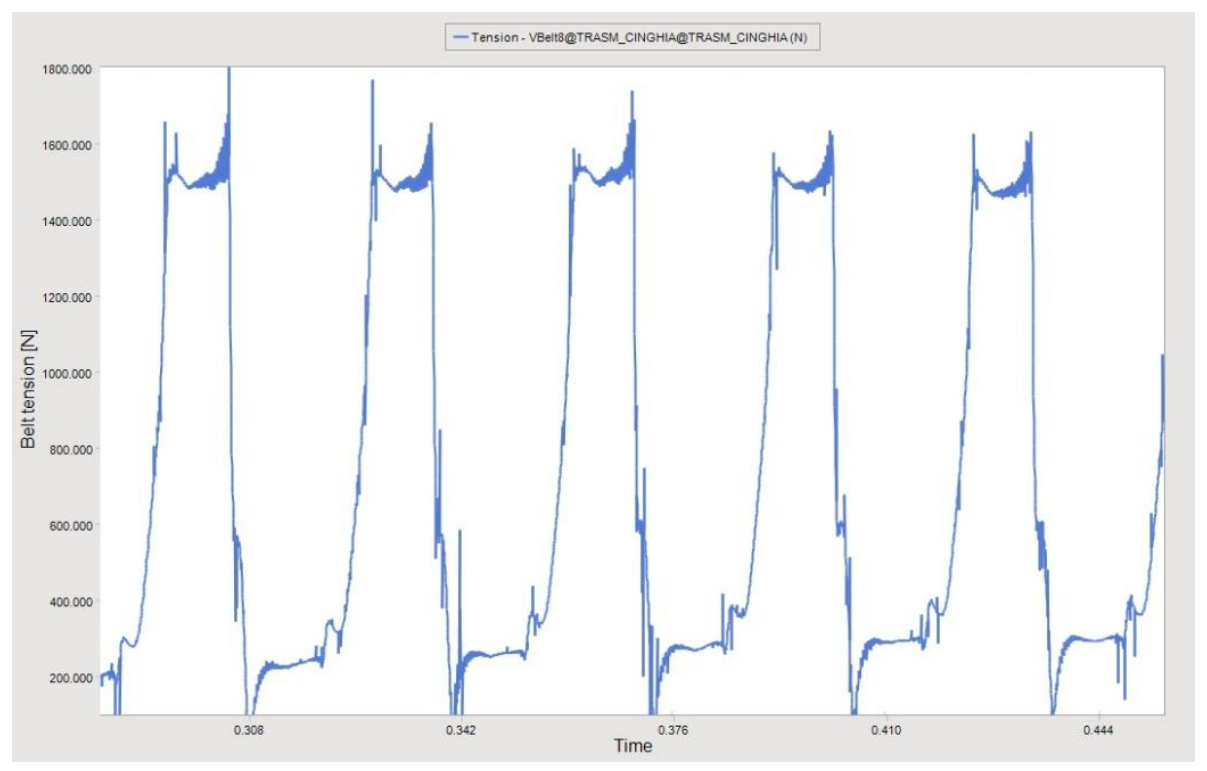

Figure 3.16: Belt tension in the maximum power test

Table 3-3: Belt tension history in maximum power

\begin{tabular}{|cccc|}
\hline Tight side & Slack side & Mean tension & Alternate tension \\
\hline $1550 \mathrm{~N}$ & $270 \mathrm{~N}$ & $910 \mathrm{~N}$ & $640 \mathrm{~N}$ \\
\hline
\end{tabular}

From Table 3-3 we can notice that the alternate tension is lowered by $19 \%$ with respect to the maximum torque condition, while the mean tension is lowered by $38 \%$.

The side pressure diagram has the same characteristics exposed for the maximum torque test. In Figure 3.17 we have reported the contact force acting on the motor pulley, whilst in Figure 3.18 the contact force in the driven pulley is shown. 


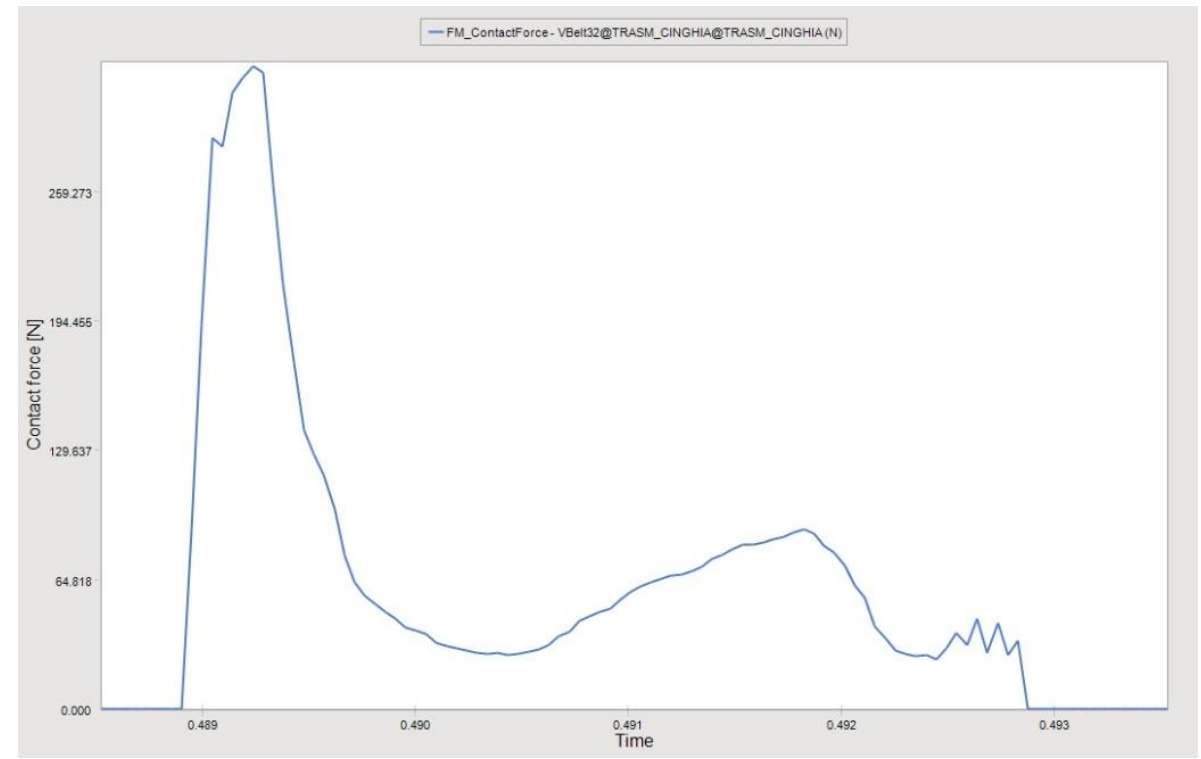

Figure 3.17: Contact force acting on the motor pulley under maximum power conditions

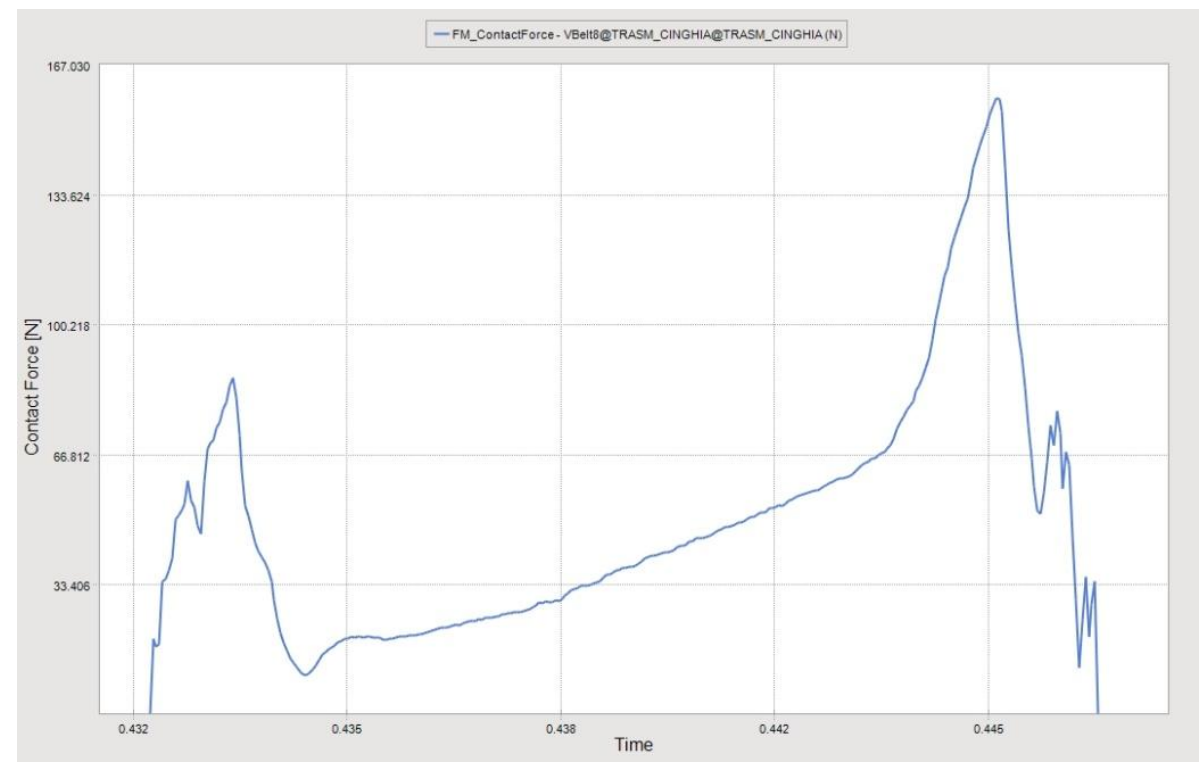

Figure 3.18: Contact force acting on the driven pulley under maximum power conditions 
In Table 3-4 are reported the data useful to interact with the fatigue diagram shown in Figure 2.3. The side contact pressure values are computed considering the area of the lateral surface of the belt elements $\left(82 \mathrm{~mm}^{2}\right)$.

Table 3-4: Data for belt durability in maximum torque

\begin{tabular}{|l|r|}
\hline Mean pressure in the driven pulley & $0.5 \mathrm{MPa}$ \\
\hline Mean pressure in the motor pulley & $1.0 \mathrm{MPa}$ \\
\hline Belt total length & $970 \mathrm{~mm}$ \\
\hline Angular velocity of the driven pulley & $915 \mathrm{rad} / \mathrm{s}$ \\
\hline Linear velocity of the belt & $49.3 \mathrm{~m} / \mathrm{s}$ \\
\hline
\end{tabular}

Given the belt length and its linear velocity, we can compute that a belt element completes $265000\left(N_{p}\right)$ revolutions per hour. Therefore we can affirm that a belt element is subdued to each load cycle $N_{p}$ times per hour. With the best fitting curve of the graph shown in Figure 2.3 we can compute the critical life times:

- $L_{d}=650 \mathrm{~h}$ for the load case related to the driven pulley

- $L_{m}=110 \mathrm{~h}$ for the load case related to the motor pulley.

Therefore, the critical number of cycles for each load case are:

- $\quad N_{d}=8.25 E+07$ for the load case related to the driven pulley

- $\quad N_{m}=1.45 E+07$ for the load case related to the motor pulley.

Considering this working condition, we can compute the damage $\left(D_{\mathrm{p}}\right)$ accumulated by the belt in one hour, with the Miner approach.

$$
D_{p}=\frac{N_{p}}{N_{d}}+\frac{N_{p}}{N_{m}}=0.010
$$

The belt tension raise in unsteady conditions can be quantified in $25 \%$ of the value measured in the steady condition, while the contact force is amplified by $15 \%$. 


\subsubsection{Over speed conditions}

This analysis has been conducted imposing a constant motor torque equal to the value of $57 \mathrm{Nm}$ at $8500 \mathrm{rpm}$. We have also imposed an opposing toque on the driven pulley equal to $47.5 \mathrm{Nm}$, as prescribed by Piaggio. As shown inFigure 3.19 , the belt tension load history relative to this load case can be schematized with the Table 3-5.

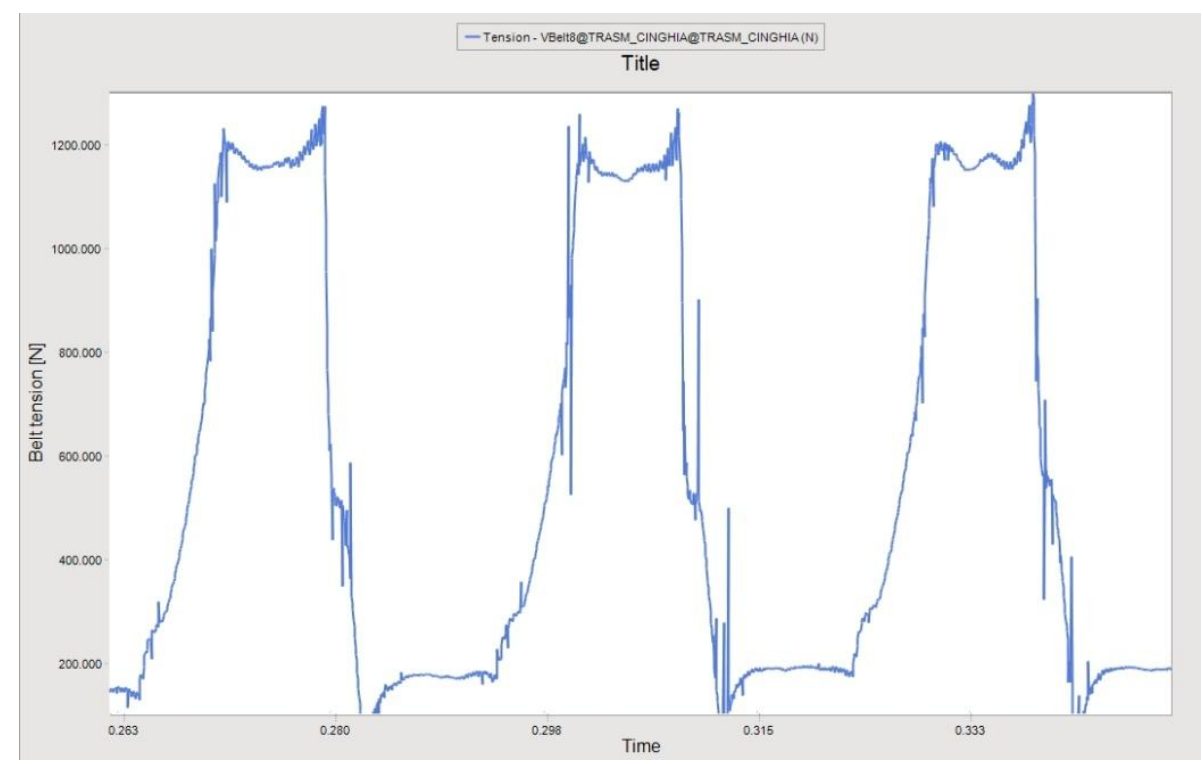

Figure 3.19: Belt tension in the over speed test

Table 3-5: Belt tension history in over speed

\begin{tabular}{|cccc|}
\hline Tight side & Slack side & Mean tension & Alternate tension \\
\hline $1200 \mathrm{~N}$ & $200 \mathrm{~N}$ & $700 \mathrm{~N}$ & $500 \mathrm{~N}$ \\
\hline
\end{tabular}

From Table 3-5 we can notice that the alternate tension is lowered by $45 \%$ with respect to the maximum torque condition, while the mean tension is lowered by $36 \%$.

The side pressure diagram has the same characteristics exposed for the maximum torque test. In Figure 3.20 we have reported the contact force acting on the motor pulley, whilst in Figure 3.21 the contact force in the driven pulley is shown. 


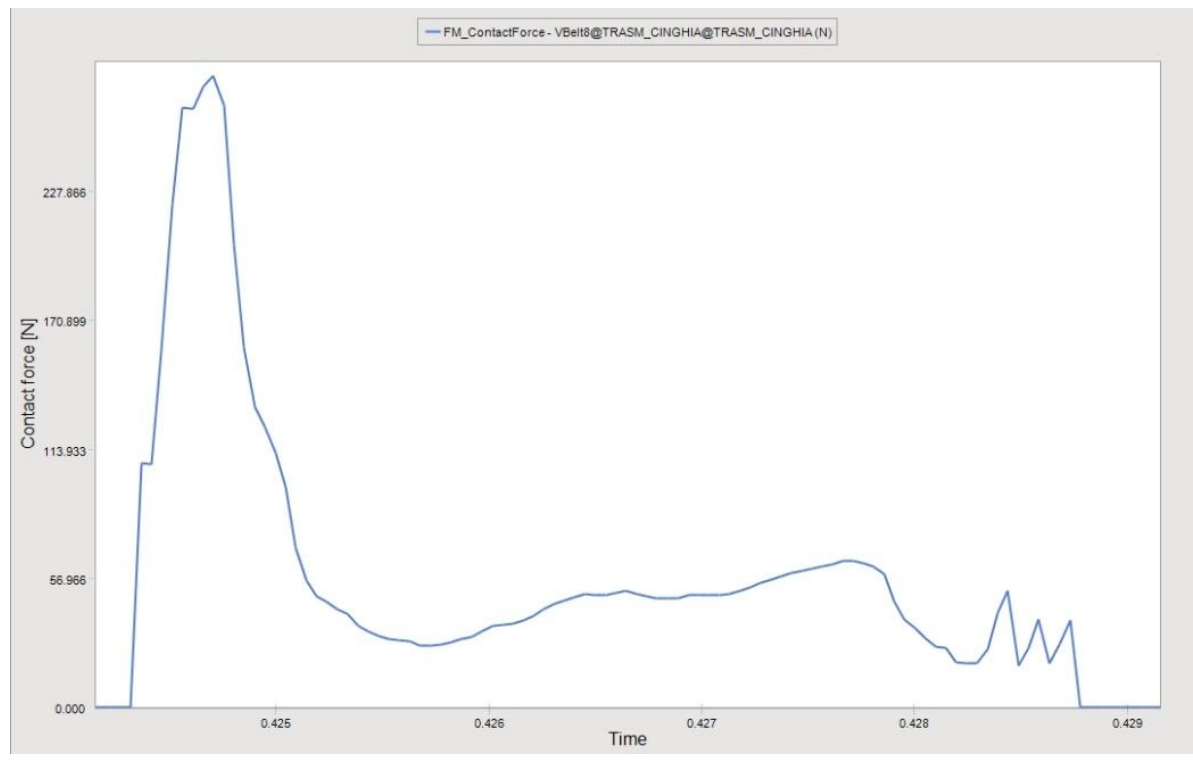

Figure 3.20: Contact force acting on the motor pulley under over speed conditions

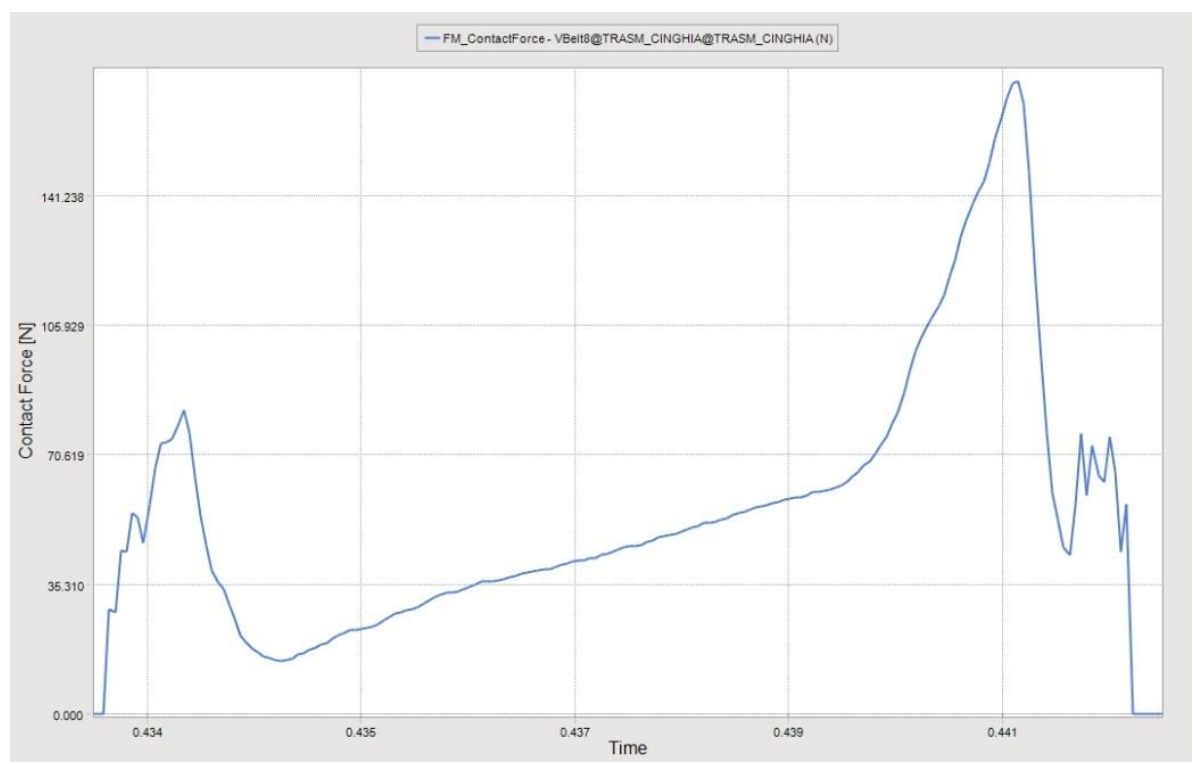

Figure 3.21: Contact force acting on the driven pulley under over speed conditions 
In Table 3-6 are reported the data useful to interact with the fatigue diagram shown in Figure 2.3. The side contact pressure values are computed considering the area of the lateral surface of the belt elements $\left(82 \mathrm{~mm}^{2}\right)$.

Table 3-6: Data for belt durability in over speed

\begin{tabular}{|l|r|}
\hline Mean pressure in the driven pulley & $0.5 \mathrm{MPa}$ \\
\hline Mean pressure in the motor pulley & $0.8 \mathrm{MPa}$ \\
\hline Belt total length & $970 \mathrm{~mm}$ \\
\hline Angular velocity of the driven pulley & $1070 \mathrm{rad} / \mathrm{s}$ \\
\hline Linear velocity of the belt & $55.9 \mathrm{~m} / \mathrm{s}$ \\
\hline
\end{tabular}

Given the belt length and its linear velocity, we can compute that a belt element completes $300000\left(N_{p}\right)$ revolutions per hour. Therefore we can affirm that a belt element is subdued to each load cycle $N_{p}$ times per hour. With the best fitting curve of the graph shown in Figure 2.3 we can compute the critical life times:

- $L_{d}=650 \mathrm{~h}$ for the load case related to the driven pulley

- $L_{m}=225 \mathrm{~h}$ for the load case related to the motor pulley.

Therefore, the critical number of cycles for each load case are:

- $\quad N_{d}=8.25 E+07$ for the load case related to the driven pulley

- $\quad N_{m}=2.90 E+07$ for the load case related to the motor pulley.

Considering this working condition, we can compute the damage $\left(D_{\mathrm{o}}\right)$ accumulated by the belt in one hour, with the Miner approach.

$$
D_{p}=\frac{N_{p}}{N_{d}}+\frac{N_{p}}{N_{m}}=0.014
$$

The belt tension raise in unsteady conditions can be quantified in $15 \%$ of the value measured in the steady condition, while the contact force is amplified by $25 \%$. 


\subsubsection{Lifetime of the belt}

Considering the results of the previous analyses, and assuming the standard mission profile described in the chapter 3.4, we can compute the estimated lifetime for the belt. Considering for instance the maximum torque conditions, using the equation (3.10) we can compute the damage accumulated in these conditions after a generic lapse of time $(\Delta t)$ spent at the engine bench.

$$
\hat{D}_{t}=D_{t} \cdot 0.05 \Delta t
$$

Computing the accumulated damage for each load case, we can define the lifetime of the belt as the $\Delta t$ needed to bring the global damage to the unitary value.

$$
\Delta t=\frac{1}{0.085 \hat{D}_{t}+0.85 \hat{D}_{p}+0.10 \hat{D}_{o}}
$$

Using the equation (3.11), the estimated lifetime for the belt in the standard engine bench test conditions is equal to 68 hours. Considering the final transmission ratio and the rear wheel diameter, we can compute that the estimated lifetime expressed in kilometers is $11500 \mathrm{~km}$, under a hypothetic use coherent with the standard mission profile. 



\section{The residual stress acting on the pulleys}

\subsection{The riveting process}

The CVT pulleys are usually made of two parts: the shaft, joined with the engine or with the clutch, and a flange, designed to couple with the transmission belt.

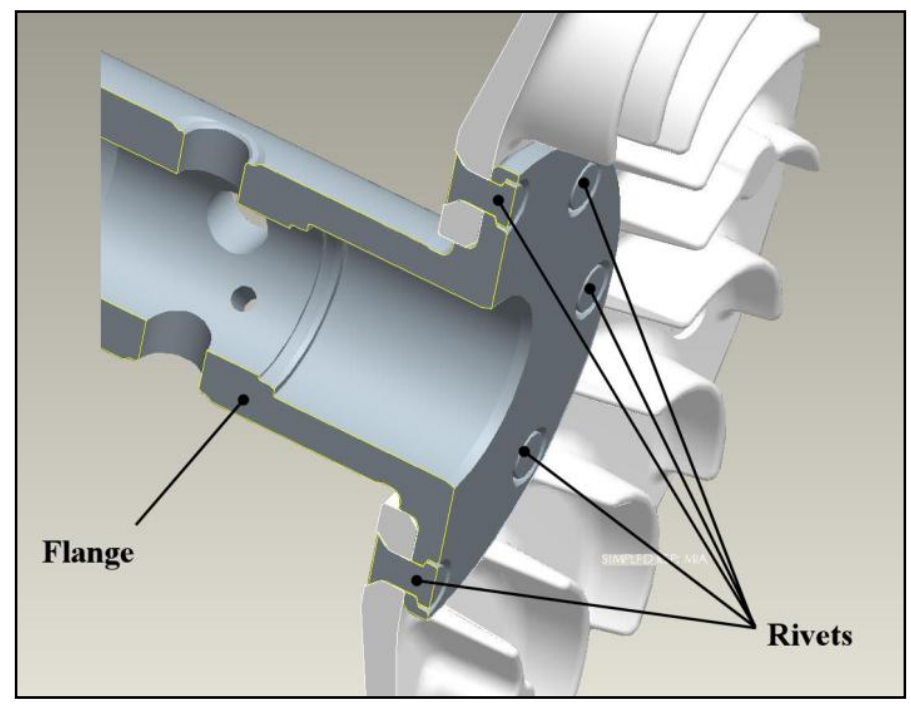

Figure 4.1: A typical CVT pulley construction

The joint between the two parts is made pressing a rivet between a fixed tool and a buckling bar, in order to generate a plastic strain in the terminal part of the rivet (driven head).

This kind of forced joint causes the raising of a stress field in the surroundings of the rivet, that keeps acting after the removal of the punching load. This residual stress affect the fatigue life of the joined parts, altering the load spectrum related to the operation of the CVT. 


\subsection{Case study}

The riveted process analyzed in this work concerns a complex, asymmetric geometry, involving the hollow shaft and the trapezoidal flange shown in Figure 4.1. Those parts are joined together by means of 10 rivets, whose holes are repeated every $36^{\circ}$ in a cylindrical system having the " $z$ " axis concentric with the shaft.

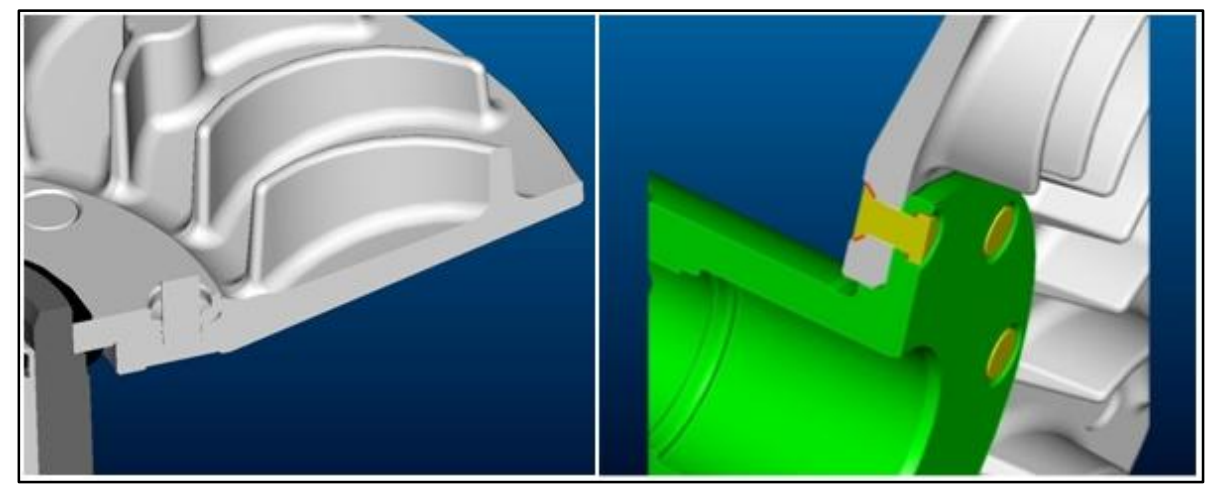

Figure 4.2: Axial section of the CVT half - pulley. Note the fins on the flange

The trapezoidal flange shows a series of fins on its surface, used to improve the heat dissipation during CVT work (see Figure 4.2). Those fins have been removed from the model, in order to reduce the complexity of the FE analysis. That simplification is justified by the distance of the fins from the riveting zone. 


\subsection{Previous works}

Due to the strong nonlinearities involved in the process, the works previously conducted in this field are based on simplified models. The main analysis techniques available in literature are the following:

\section{- Analytic model \\ - Experimental measures \\ - FEM analysis.}

The analytic model proposed by Park and Atluri [21] is based on the assumption that a deformed rivet applies a radial pressure $p$ on the cylindrical surface of a hole realized on a sheet metal with infinite extension and with an elastic perfectly plastic behavior. The stress field around the hole is given by the equations (4.1) and (4.2):

$$
\begin{aligned}
& \sigma_{r}=-p \cdot\left(\frac{r}{R_{0}}\right)^{-2} \\
& \sigma_{\vartheta}=p \cdot\left(\frac{r}{R_{0}}\right)^{-2}
\end{aligned}
$$

Where $\sigma_{r}$ and $\sigma_{\vartheta}$ are respectively the radial and circumferential stresses, $r$ is the distance from the rivet axis to the measuring point and $R_{0}$ is the rivet radius. If the pressure $p$ causes a plastic deformation in the sheet metal, the stress distribution can be expressed with the equations (4.3) and (4.4):

$$
\begin{aligned}
& \left\{\begin{array}{c}
\sigma_{r}=-p+\sigma_{y} \ln \left(\frac{r}{R_{0}}\right) \quad R_{0} \leq r \leq r_{y} \\
\sigma_{r}=-\frac{\sigma_{y}}{2}\left(\frac{r_{y}}{r}\right)^{2} \quad r>r_{y}
\end{array}\right. \\
& \left\{\begin{array}{cc}
\sigma_{\vartheta}=\left(\sigma_{y}-p\right)+\sigma_{y} \ln \left(\frac{r}{R_{0}}\right) & R_{0} \leq r \leq r_{y}
\end{array}\right. \\
& \sigma_{\vartheta}=\frac{\sigma_{y}}{2}\left(\frac{r_{y}}{r}\right)^{2} \quad r>r_{y}
\end{aligned}
$$

Where $r_{y}$ is the radius of the plastic region and $\sigma_{y}$ is the yield stress of the material. In the model proposed by Park and Atluri, the residual stresses acting on the sheet metal are given by the subtraction of the elastic stress (given by the equations (4.1) and (4.2)) from the plastic stress field (given by (4.3) and (4.1)). This model introduces some hard approximations, also underlined by the FEM analysis 
conducted by Müller [22], since it excludes the possibility of work hardening of the material, and omits the thickness variations of the sheet metal; the model also omits the stress field acting on the sheet metal in the diction of the rivet axis, caused by the deformation of the driven head.

Langrand and Alii [23] have done an experimental investigation on the residual stress field acting in rivets and plates, applying radial lines of stress gauges. The tests have shown that the strain levels in the vicinity of the punching, reaches the level of $20 \%$ at $0.5 \mathrm{~mm}$ from the punching edge. This method, as stated by its authors, could be used to develop and validate complex Finite Element models, but shows some limits, like the high cost of the experimental campaign and the impossibility of stress measurements in the axial direction of the rivet.

Fitzgerald and Cohen [24] proposed a X-Ray technique to visualize the residual stress field in riveted plates, scanning the specimen on both faces (entrance and exit of the rivet). The analysis showed compressive stresses around the hole of the plate, with values higher on the entrance face rather than the exit one, highlighting the variation of the stress field in the direction of the thickness, but avoiding the determination of its rate of change.

Another experimental campaign was conducted by Ryzhova [25], which measured with an ultrasonic approach the radial stress field along the height of the rivet, stating a linear correlation between the mean amplitude of the signal reflected by the rivet material and the average residual stress. No data were obtained in the circumferential direction.

As shown by the previous examples, the complexity of the riveting process make the characterization of the residual stress field too hard to be explained in an analytical way. Furthermore, the mentioned experimental methods could provide some limited information about the real state of stress of both the rivet and the flanges. The Finite Element method offers a more effective approach to explore the state of stress in such conditions, since it allows to compute the stress acting on each part of the assembly without hard assumptions and avoiding perturbations in the stress field.

Most of the FE analysis performed in the past to describe the riveting process are based on two dimensional approximations of the problem, due to the high non linearity of the problem. Slater [26] and Müller [22] analyzed a two dimensional model of the process, implementing a non linear contact algorithm, but omitting the work hardening of the material, that was introduced in the model by Szolwinski and Farris [27] in a succeeding analysis. These studies showed that the riveting process generate a residual stress gradient along the thickness of the flanges, with a circular field of compression stress around the hole, and a field of circumferential traction stress that recedes from the hole with the raising of the riveting force. 
The analysis conducted by Blanchot and Daidie [28] is the first non two dimensional model carried out in the riveting process analysis. It refers to a riveted joint achieved imposing a sinusoidal motion law to the steel punch body.

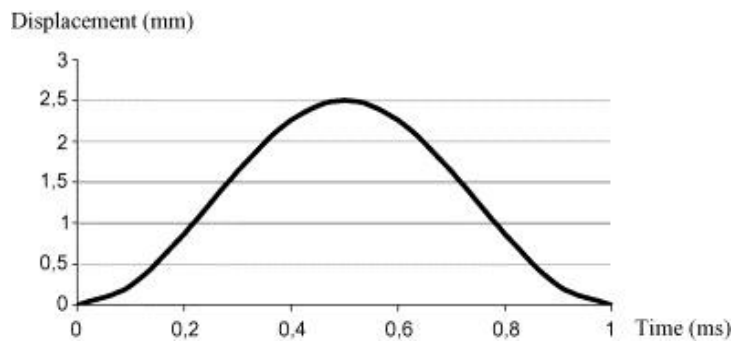

Figure 4.3: Punch body displacement law in [28]

The displacement controlled riveting process could bring to error in evaluating the actual riveting force, since it imposes a strain rate to the materials. More accurate results could be achieved with a force controlled process.

Each body involved in the simulation has been modeled with an elastoplastic correlation between stress and strain, obtained with standardized traction tests. The authors have conducted three different simulations, considering at the beginning a simple two-dimensional model ("Slice Model"), then two solid revolution of the plane sketch of the assembly: one ("Axisymmetric Model") with a 15 degrees rotation and the last with a 180 degrees rotation ("Symmetric Model").

The models realized by Blanchot and Daidie have been improved by Atre in succeeding analyses [29].

The Piaggio Company has realized in the past an analysis of the riveting process involved in the assembly of the half pulley used in its scooters [30], building two FE models of the system, one for each of the parts of the cross section shown in Figure 4.4. 


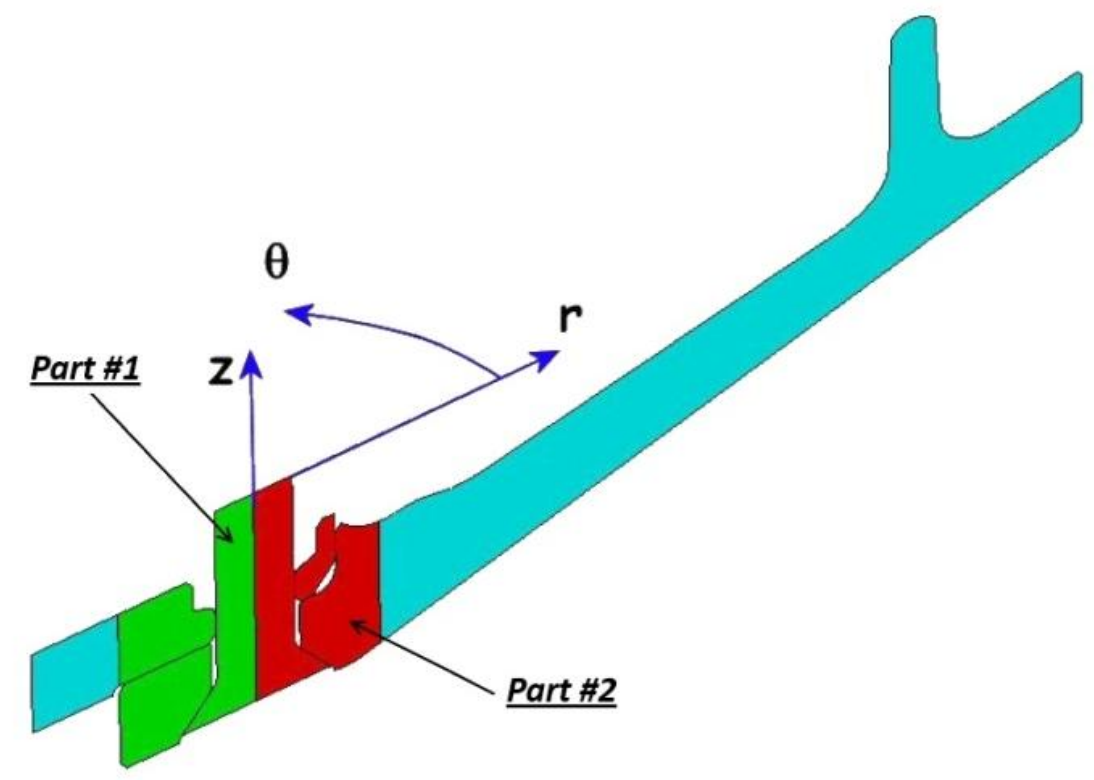

Figure 4.4: The riveting model described in [30]

In this analysis, called $\mathbf{u}_{\mathbf{1}}$ and $\mathbf{u}_{\mathbf{2}}$ the displacement vectors computed for the generic point of the two axisymmetric models (part \#1 and \#2), with reference to the coordinate system defined in Figure 4.4, we can write:

$$
\begin{aligned}
& \mathbf{u}_{1} \neq \mathbf{u}_{1}(\theta) \\
& \mathbf{u}_{\mathbf{1}}=\mathbf{u}_{\mathbf{1}}(r, z) \\
& \mathbf{u}_{\mathbf{2}}=\mathbf{u}_{\mathbf{2}}(r, z)
\end{aligned}
$$

Let $\mathbf{u}$ be the displacement vector of the point $P\left(r_{0}, \theta, z_{0}\right)$ of the real component; in the work presented has been assumed that:

$$
\mathbf{u}=\frac{\pi-\theta_{0}}{\pi} \mathbf{u}_{\mathbf{2}}\left(r_{0}, z_{0}\right)+\frac{\theta_{0}}{\pi} \mathbf{u}_{\mathbf{1}}\left(r_{0}, z_{0}\right)
$$

The work showed that the part of the pulley near the external portion of the rivet seat (Part \#2) is subject to a compressive stress field, which doesn't worsen the fatigue conditions subsequently induced by the working conditions. The 
opposite has be observed for the internal side of the rivet seat, that is the area where the Piaggio Company have registered some failure of the pulley.

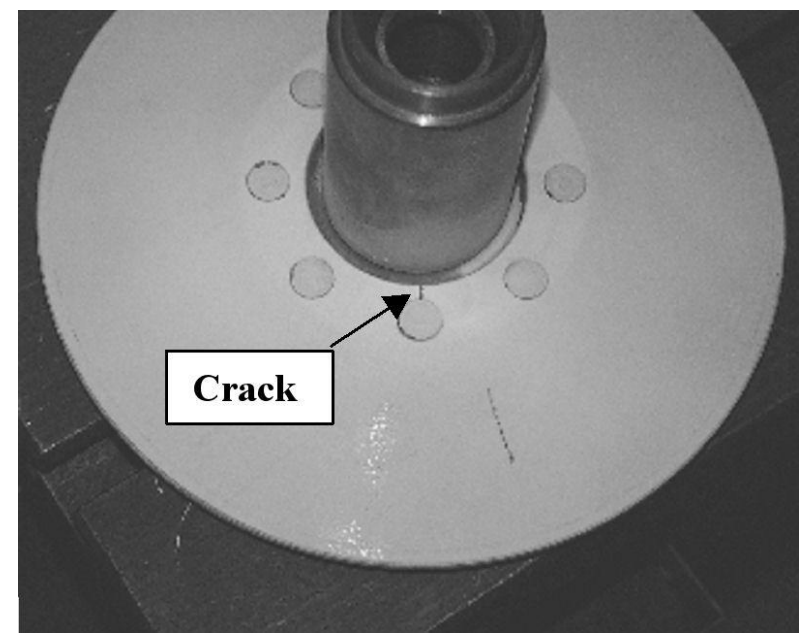

Figure 4.5: Typical location of cracks in the half-pulley assembly

The studies of Blanchot, Daidie and Atre have shown a good correlation with the experimental data, but are limited to simple geometries, with an axis of symmetry. The work of Dal Grande and Testi has considered a non axisymmetric geometry, but introduces an arbitrary interpolation of two-dimensional studies. The aim of this part of our work is to build up a complete model of the parts involved in the process, in order to evaluate the actual residual stress field of a complex geometry subject to the riveting process. 


\subsection{The complete FE model of the assembly}

\subsubsection{Discretization of the geometry}

The mesh of the half pulley has been obtained pointing out that the assembly of rivet, flange and shaft could be represented by an elementary set recurring every 36 degrees around the axis of rotation of the shaft. This set is then provided of a symmetry plane containing the axis of the hole. Under these statements, it is possible to discretize a 18 degrees portion of the assembly and then replicate the mesh to obtain the full model.

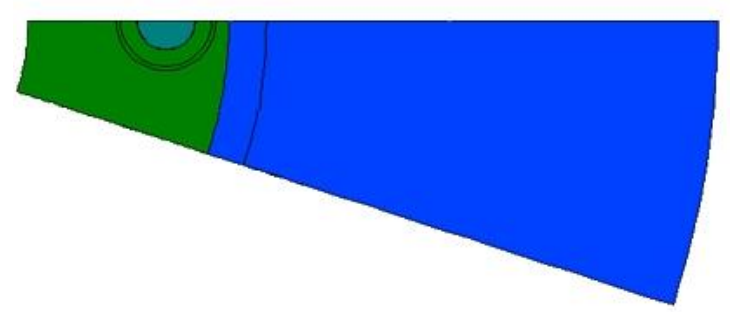

Figure 4.6: The 18 degrees portion of the elementary set of the half-pulley

We have chosen to discretize the geometry only with hexahedral 8-nodes elements; this choice has been adopted since the hexahedral elements show a higher order form function compared to the tetrahedral ones, with a more efficient algorithm of displacement estimation. Moreover, the hexahedral elements allow to discretize the volume with a lower distortion, with the respect of their native cubic shape. The final discretization is composed of 118630 elements, with 31770 elements in the rivet, 37580 in the shaft and 49280 in the flange. The density of elements is not uniform: the region around the rivet is more filled than the other parts of the assembly, in order to keep low the total amount of elements, still ensuring a high level of accuracy. This result has been achieved using both the free and mapped mesh approach, to adapt the element characteristic to the geometry of the belt. 


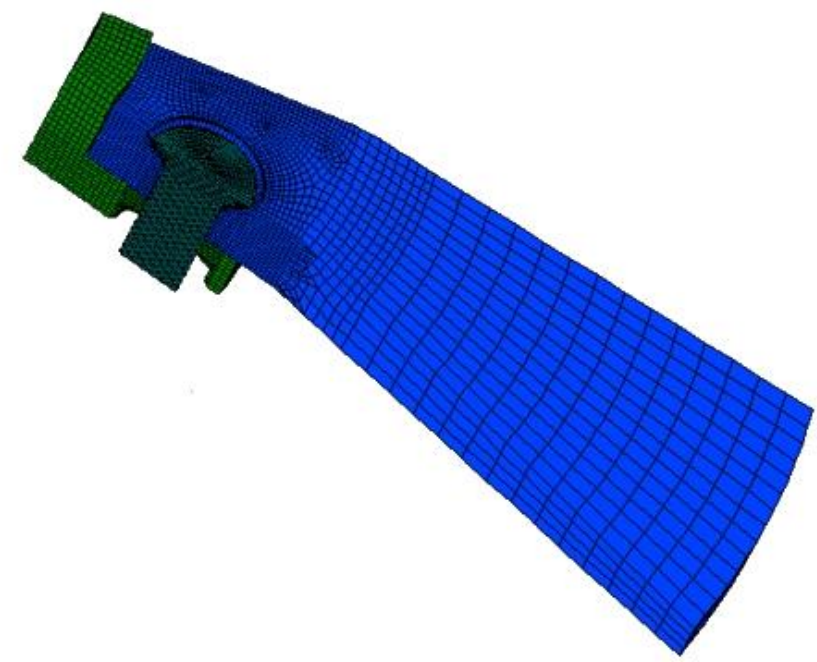

Figure 4.7: Mesh of the elementary set

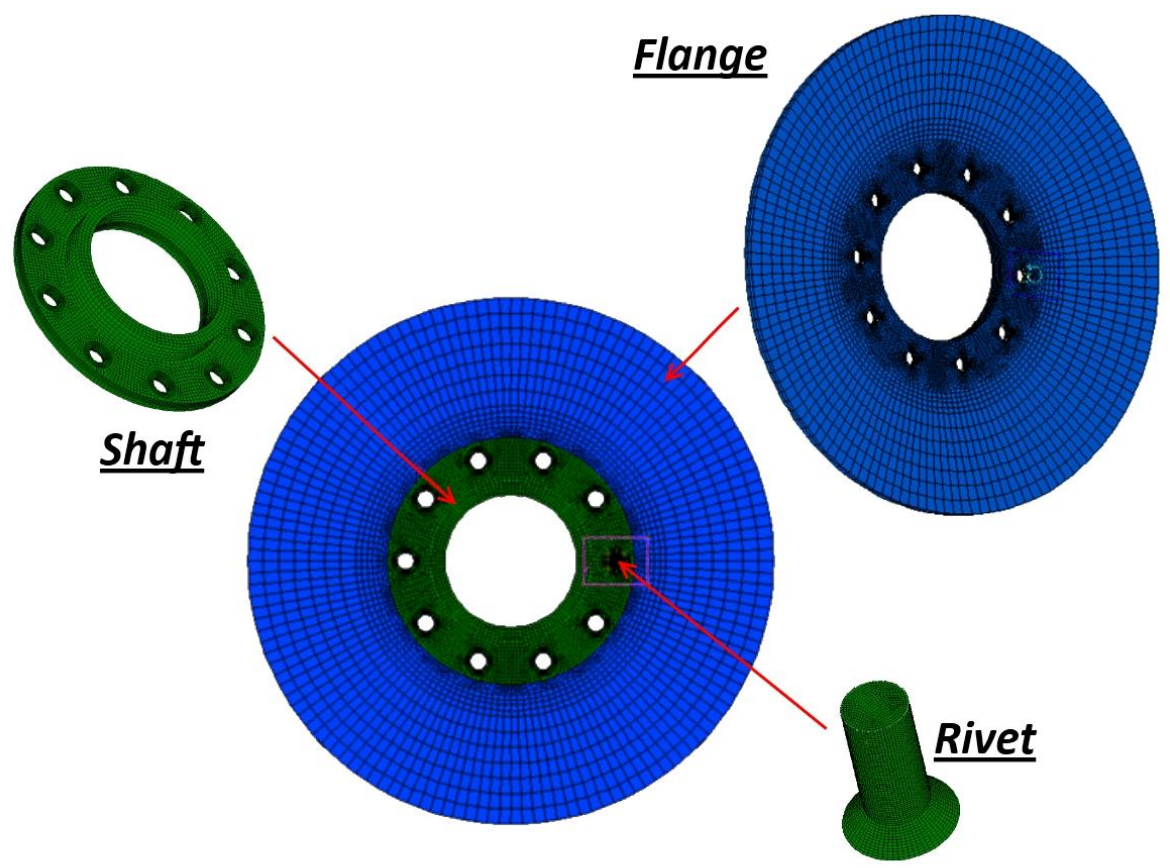

Figure 4.8: The model completely meshed 


\subsubsection{Material properties}

We have modeled the materials involved in the process with a linear work hardening law, approximating the actual relation between stress and strain with a bilinear function like the one shown in Figure 4.9.

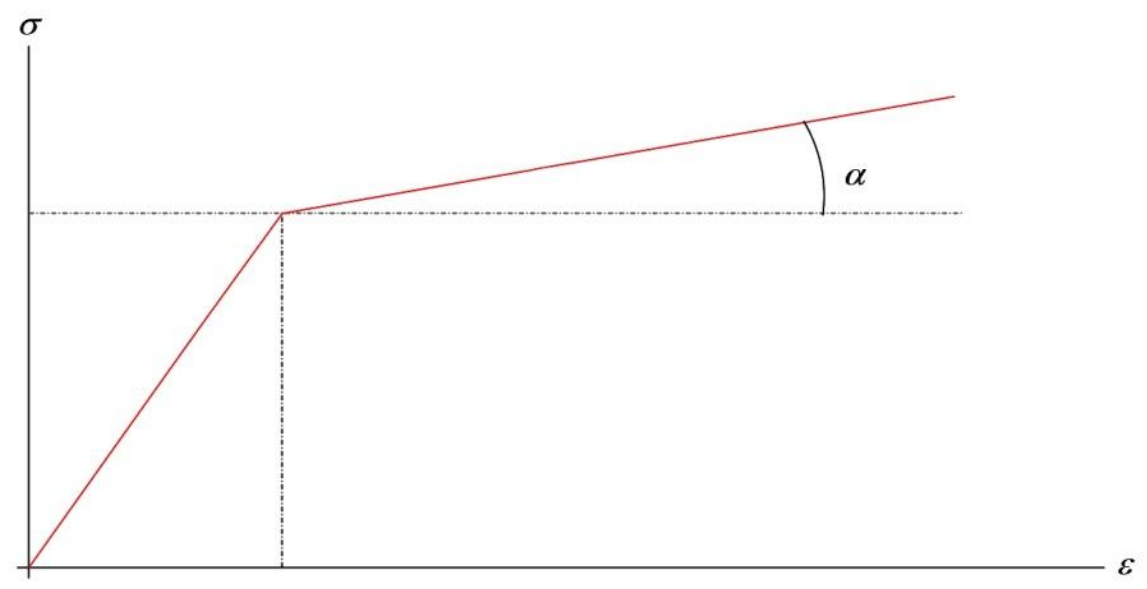

Figure 4.9: Bilinear stress-strain correlation

\subsubsection{The rivet material}

In our application, the rivet is made of C40 steel (UNI designation). From measurements performed by Piaggio, the rivet shows a yield stress of $550 \mathrm{MPa}$ and an ultimate strength of $600 \mathrm{MPa}$. The ultimate elongation is $22 \%$, and the Young modulus is $210000 \mathrm{MPa}$. These values are referred to the unloaded area of the section. To convert the nominal stress and strain values to the actual one, we can use the well known relations (4.9) and (4.10).

$$
\begin{aligned}
& \sigma_{u}=S_{u}\left(1+e_{u}\right) \\
& \varepsilon_{u}=\ln \left(1+e_{u}\right)
\end{aligned}
$$

Where:

- $\sigma_{u} \quad$ is the actual ultimate strength

- $S_{u} \quad$ is the nominal limit strength (600MPa)

- $\varepsilon_{u} \quad$ is the actual ultimate deformation

- $e_{u} \quad$ is the nominal ultimate deformation (0.22). 
Therefore, the actual ultimate stress and strain are given by (4.11) and (4.12).

$$
\begin{aligned}
\varepsilon_{u} & =0.199 \\
\sigma_{u} & =732 \mathrm{MPa}
\end{aligned}
$$

As far as it what concerns the yield limit, the deformations of the section area are negligible, so we can express the actual stress and strain with the (4.13) and (4.14) relations.

$$
\begin{aligned}
& \sigma_{y}=S_{y}=550 \mathrm{Mpa} \\
& \varepsilon_{y}=\frac{S_{y}}{E}=0.00262
\end{aligned}
$$

\subsubsection{The shaft material}

The shaft of the half pulley is made of Fe410 steel (Piaggio normative[31]), with the following behavior:

$\begin{array}{ll}\text { - Young modulus: } & 210000 \mathrm{MPa} \\ \text { - Yield nominal strength: } & 255 \mathrm{MPa} \\ \text { - Ultimate elongation: } & 22 \% \\ \text { - Ultimate strength: } & 410 \mathrm{MPa}\end{array}$

The actual values of stress and strain are given by (4.15), (4.16) and (4.17).

$$
\begin{aligned}
& \varepsilon_{u}=0.199 \\
& \sigma_{u}=500 M P a \\
& \varepsilon_{y}=0.00121
\end{aligned}
$$




\subsubsection{The flange material}

The flange material is made with a die cast process of the "Silafont 36 " aluminum alloy (commercial name, conventional designation: AlSi9MgMn). Due to the strong influence of the casting parameters on the behavior of the material, we have chosen to measure the characteristic of the alloy with dedicated traction tests done on dissection specimens (obtained from a die cast product). In chapter 4 we have reported the test conducted on those specimens to measure the fatigue strength of the material, whilst here only the static tests are described.

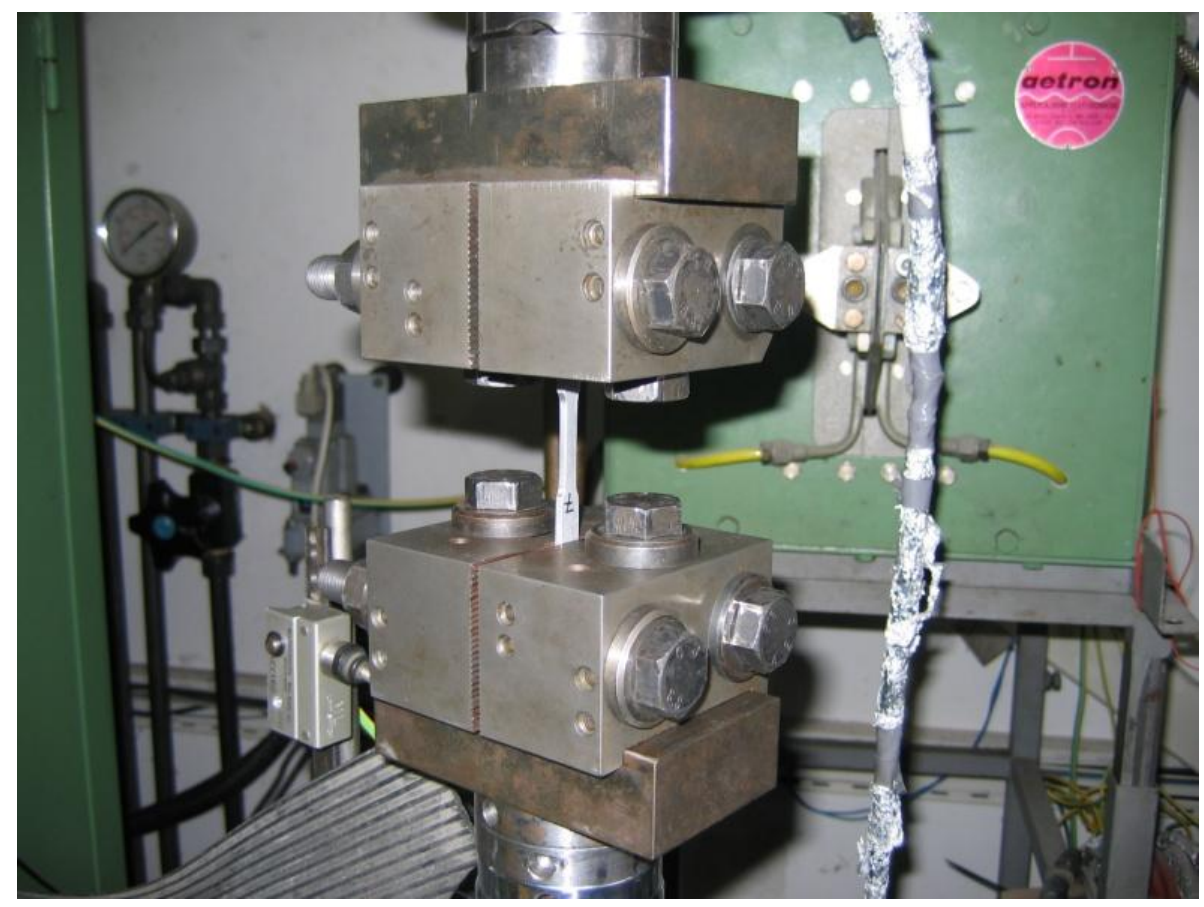

Figure 4.10: The test bench setup

The static traction test has shown the characteristic reported on Figure 4.11, where the red line has been used to compute the yield strength of the material, imposing a total plastic strain equal to the $0.2 \%$ of the initial length $(100 \mathrm{~mm})$. 


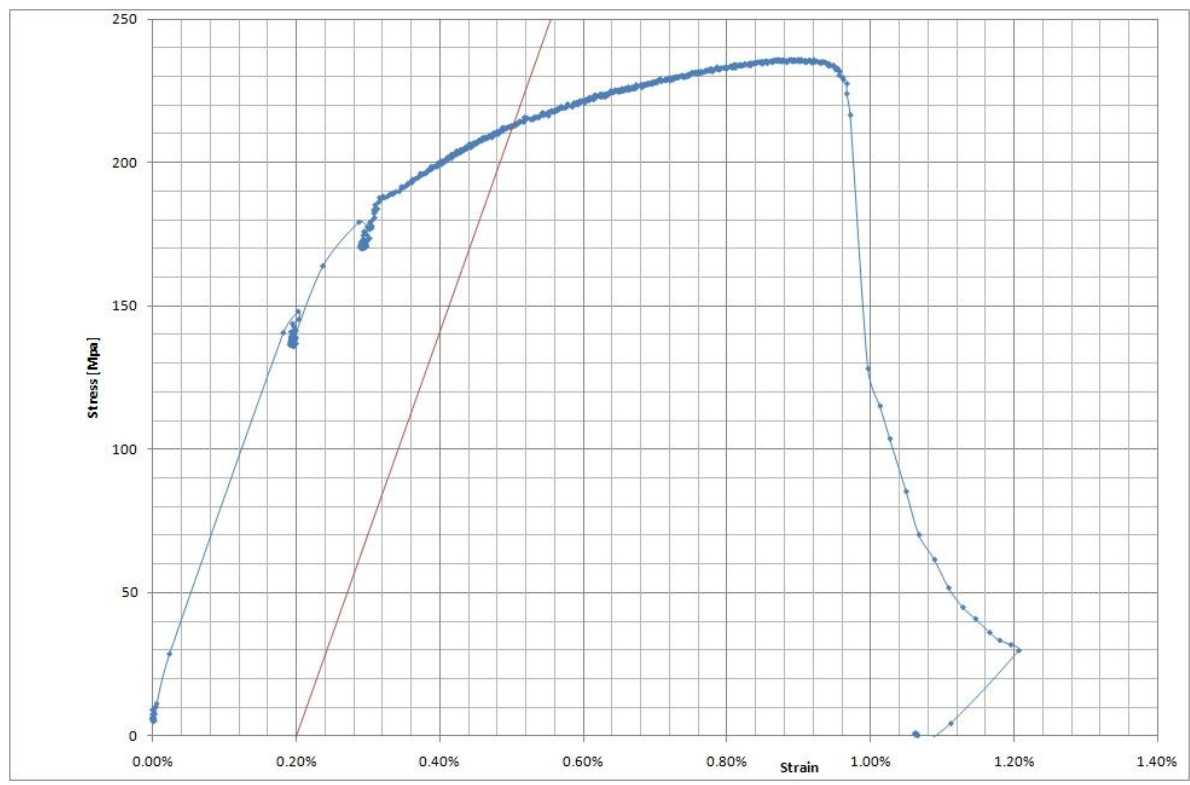

Figure 4.11: The static traction test performed on the flange material

The results of the traction test are reported in the following list:
- Young modulus:
$70500 \mathrm{MPa}$
- Yield nominal strength:
$210 \mathrm{MPa}$
- Ultimate elongation:
$9 \%$
- Ultimate strength:
235MPa.

The actual values of stress and strain are given by (4.15), (4.16) and (4.17).

$$
\begin{aligned}
& \varepsilon_{u}=0.0862 \\
& \sigma_{u}=229 M P a \\
& \varepsilon_{y}=0.00298
\end{aligned}
$$




\subsubsection{Contact formulation}

In the real world, on the parts of the assembly act mutual contact forces that evolve in the perpendicular and tangential directions of the contiguous surfaces, with the last ones generated by the friction coefficient. In the numerical model, we have modeled those parts with finite elements reflecting the actual material an geometric properties, and in a first attempt we have spread a set of contact elements on the boundary surface of every contiguous body.

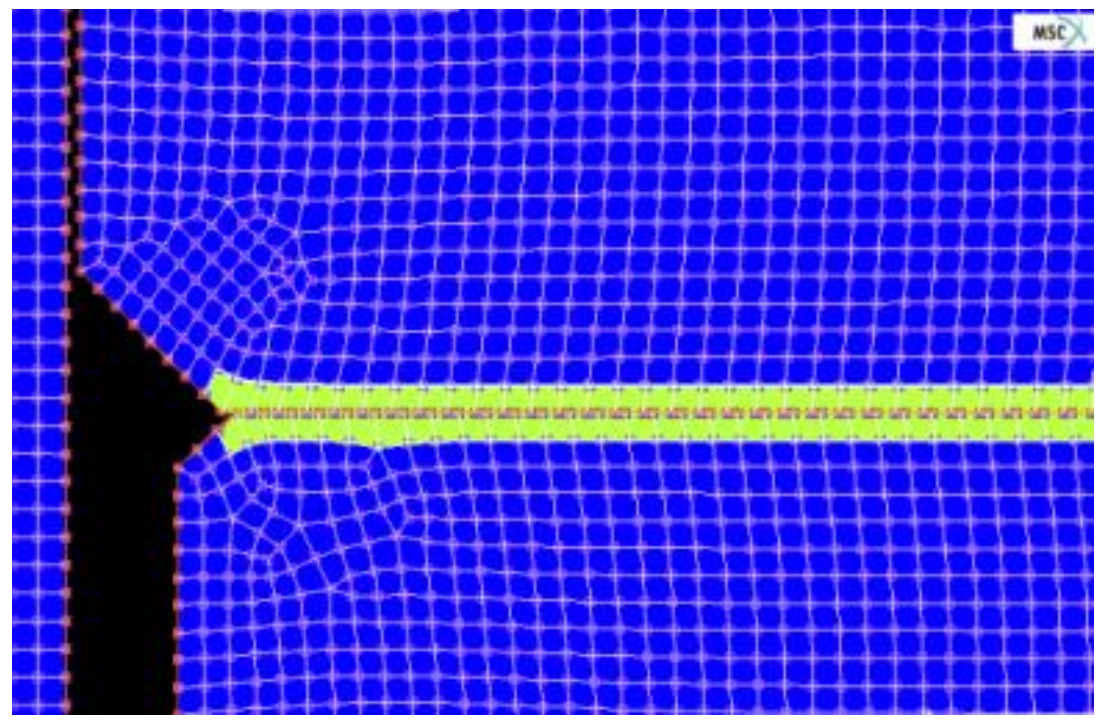

Figure 4.12: An example of the definition of the contact elements

Since this method has shown a hard computational cost, we have chosen to adopt an analytical formulation of the contact surfaces. Hence, we have generated the interpolating surfaces of the contiguous faces of each body, defining the discontinuity in the surface formulation with the edges of the bodies. This method has yielded a significant saving in simulation time.

We have then generated a contact table of our problem with that approach, assuming the following friction coefficients [32]:

- Steel on steel (shaft on rivet):

0.75

- Steel on aluminum (flange on rivet, flange on shaft):

0.6

The punch body has been modeled with a rigid body: we have assumed for the contact formulation between that part and the rivet the same friction coefficient assumed for the steel on steel contact. 


\subsubsection{Boundary conditions}

We have chosen to operate the punch body controlling the punching force, and so applying the riveting force to the punch body, that has been modeled with a rigid plane.

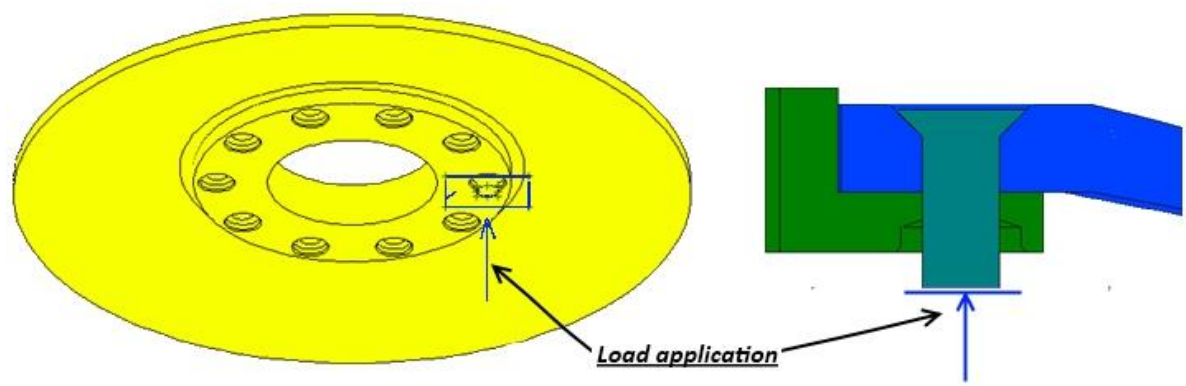

Figure 4.13: The force controlled punch body actuation

Basing on the Piaggio specifications, we have adopted a punching force equal to $56500 \mathrm{~N}$, imposing a gradual loading, in order to avoid convergence issues. Therefore, the load has been applied in 0.5 seconds with a linear law. After this interval, we have imposed the immediate removal of the contact interaction between the punch body and the rivet, in order to measure only the residual stress field, excluding the elastic spring back.

The upper surface of the pulley has been joined to the ground, in order to set to zero the displacements of each node of that side of the flange, the shaft and the rivet. 


\subsubsection{Results}

During the compression, the rivet gets deformed in the radial direction too, assuming the typical barrel shape, due to the friction forces shared with the punch body. The maximum radial deformation is close to $1 \mathrm{~mm}$, hence close to the $28 \%$ of the original radius $(3.5 \mathrm{~mm})$. From Figure 4.14 can be noticed that the deformation of the rivet is not symmetric with the respect of the axis of the rivet itself, reaching the value of $0.96 \mathrm{~mm}$ on the outer side and the value of $0.78 \mathrm{~mm}$ on the inner one.

In Figure 4.15 we have reported the deformation history of the assembly. As highlighted by the first image, due to the linear time law of load application, the rivet shows some plastic deformations at the time $0.1 \mathrm{~s}$. In the last frame, which refers to the release of the punching force, can be noticed that the rivet has been permanently deformed by $2.1 \mathrm{~mm}$. This result matches the experimental evidence supplied by Piaggio.
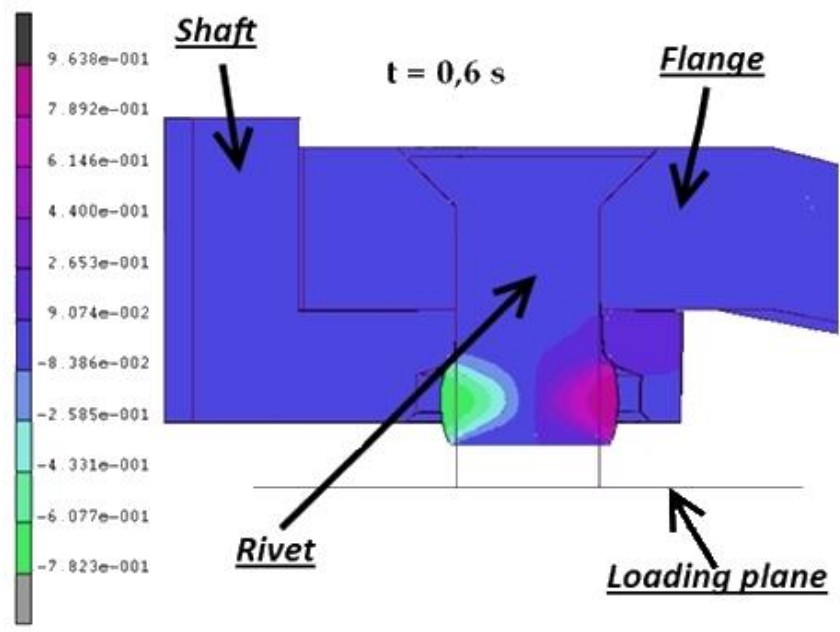

Figure 4.14: Radial deformation 


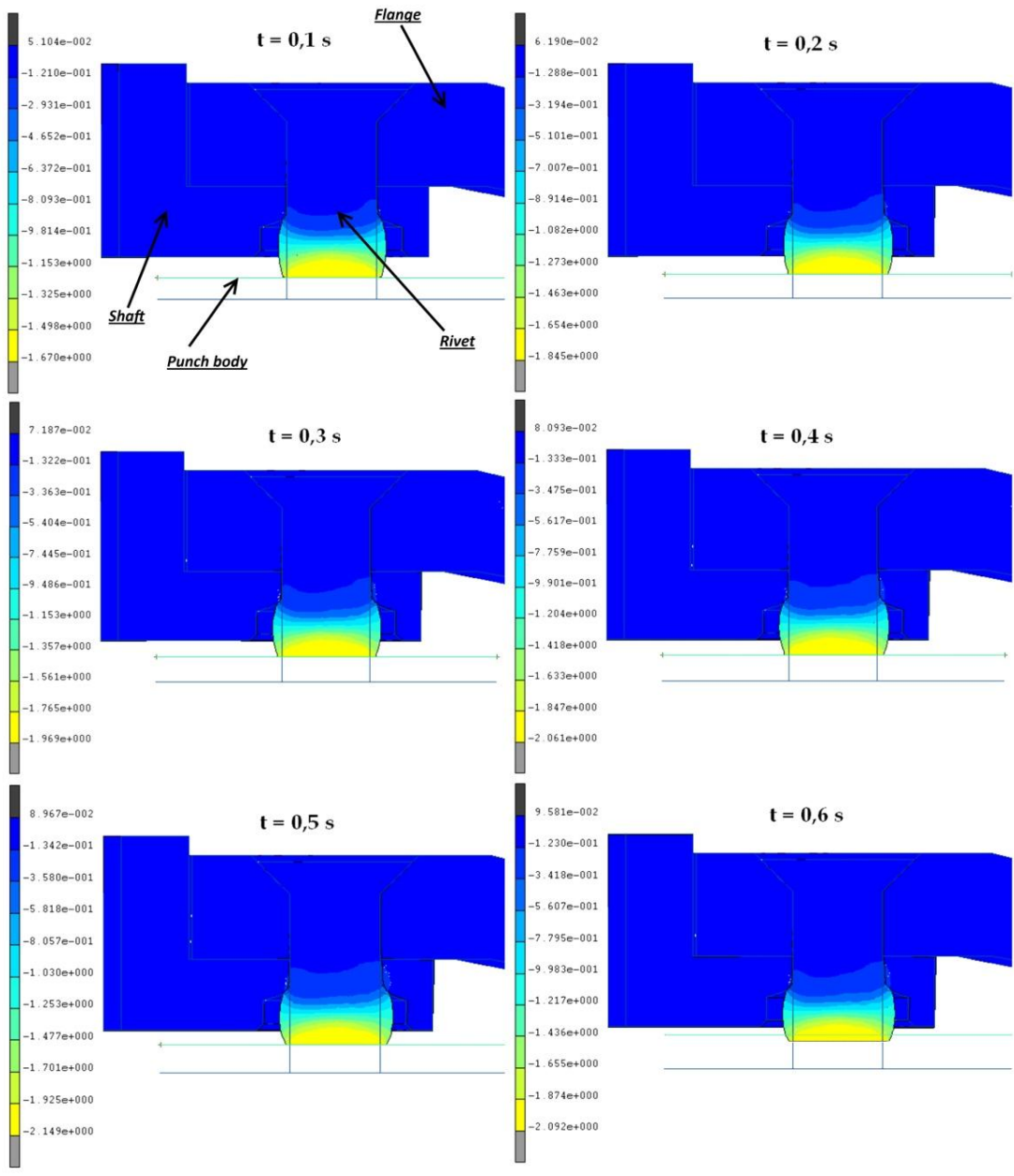

Figure 4.15: Deformation history of the assembly

From the post processing of the results can be noticed that the rivet is not the only part subdued to plastic deformations. The Figure 4.16 shows the presence of plastic fields in the shaft, at the intersection between the cylindrical surface and the conical seat of the rivet head. 

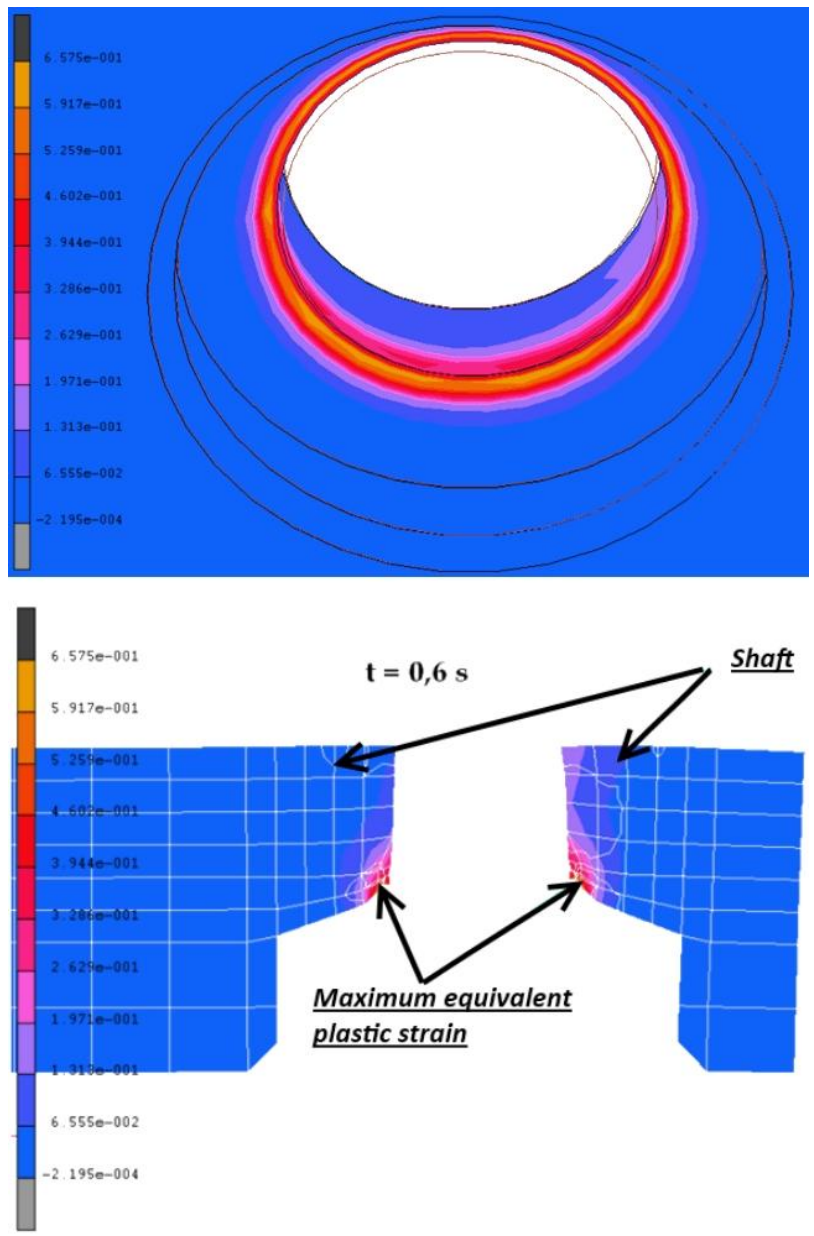

Figure 4.16: Plastic strain in the shaft

The equivalent plastic strain (given by the composition of the three principal components of strain) is uniformly distributed in the circumferential direction, and reaches the maximum value of $0.66 \mathrm{~mm}$. As shown in Figure 4.17 , the plastic region is much more limited in the flange, where the maximum value of plastic strain is $0.03 \mathrm{~mm}$. 

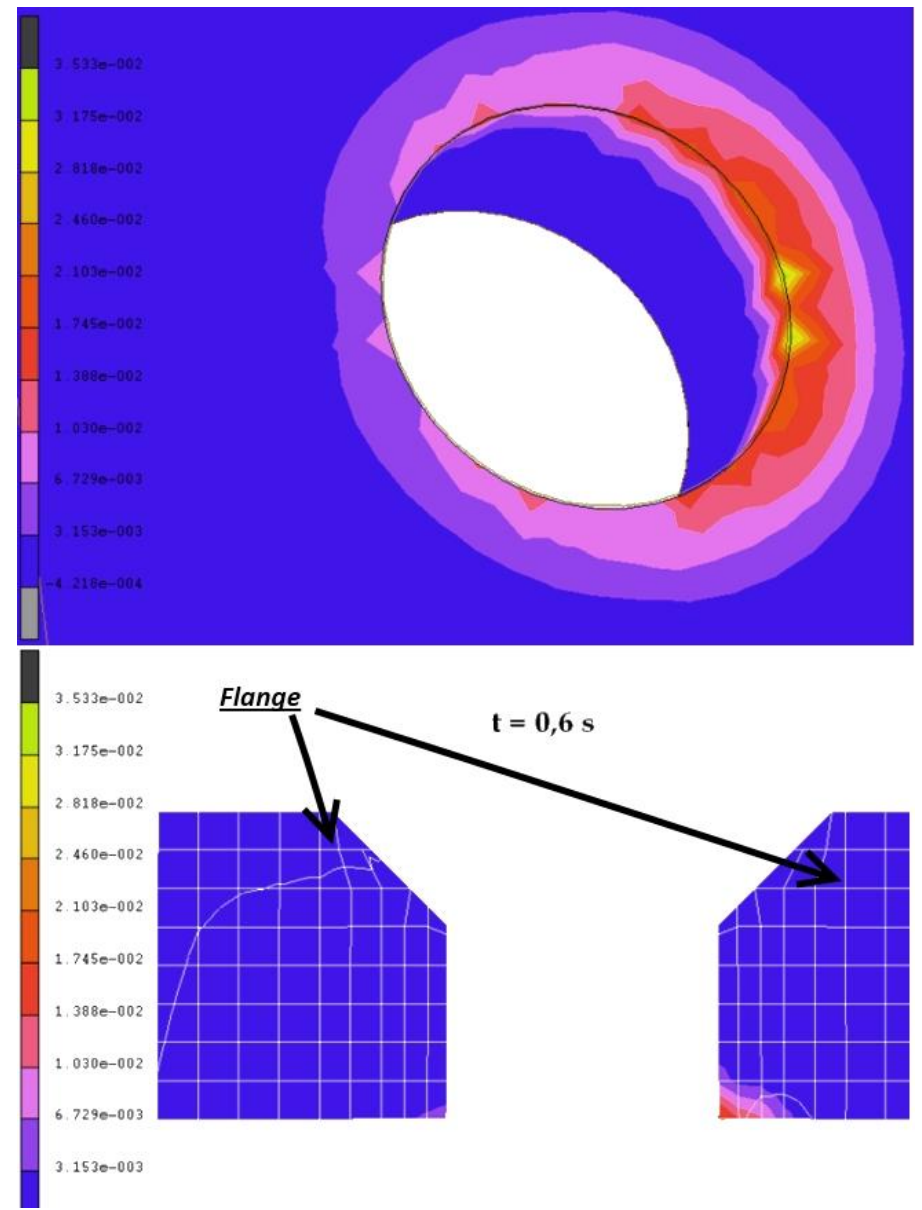

Figure 4.17: Plastic strain on the flange 


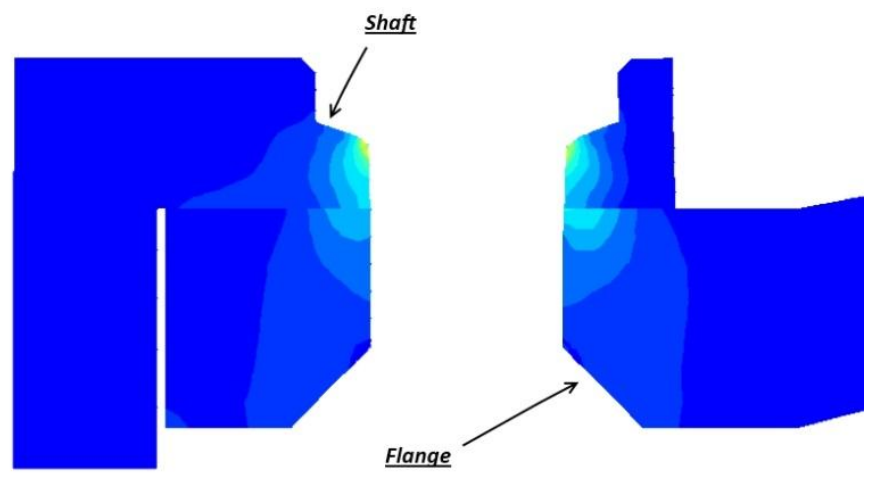

Figure 4.18: Minimum principal stress field

In Figure 4.18 it is reported the field of the minimum principal component of stress acting on the flange and on the shaft. In the surroundings of the hole, this component assumes the radial direction with reference to the rivet axis. The flange is stressed in this direction only in compression (negative values of $\left.\sigma_{\min }\right)$, with a maximum value of $210 \mathrm{MPa}$ in the outer side of the rivet seat. Even in the shaft we can see a region subdued to high compressive stresses, with a maximum value of $650 \mathrm{MPa}$ where the friction force acting in the interface between the flange and the shaft evolves on an edge. 


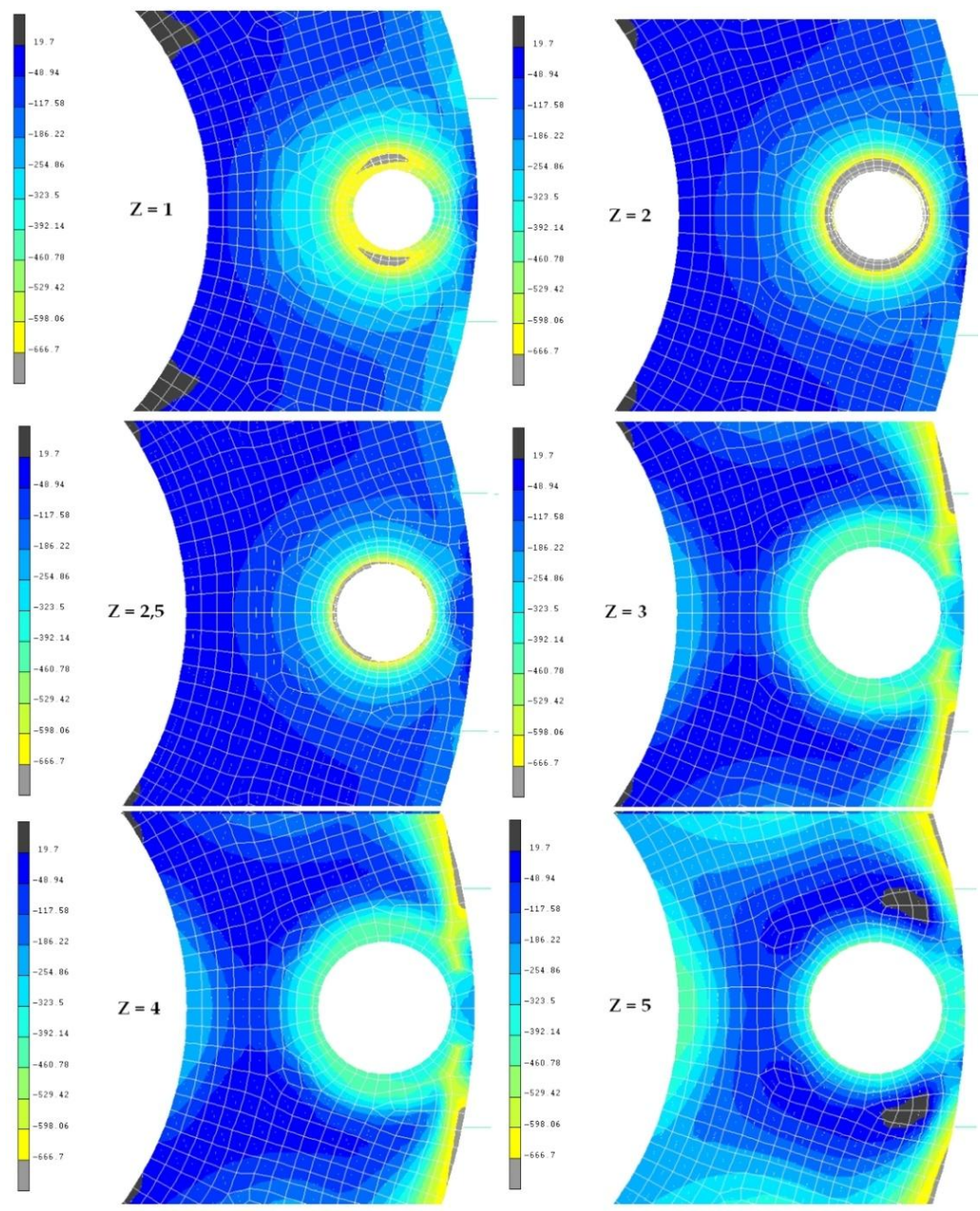

Figure 4.19: Minimum principal stress field in the shaft. The measure of " $z$ " points out the distance of the section plane from the surface of contact with the flange.

In Figure 4.19 it is presented the minimum principal stress filed acting on the shaft. To plot that image we have generated six section planes perpendicular to the axis of rotation of the pulley. The more stressed region in the radial direction is the cylindrical surface of the hole (form $z=0$ to $z=2.5$ ), where the material of the shaft withstands to the radial deformation of the rivet. On the free surface of the shaft $(z=5)$ we can notice two regions where the minimum principal stress reaches a positive maximum, equal to $20 \mathrm{Mpa}$. 


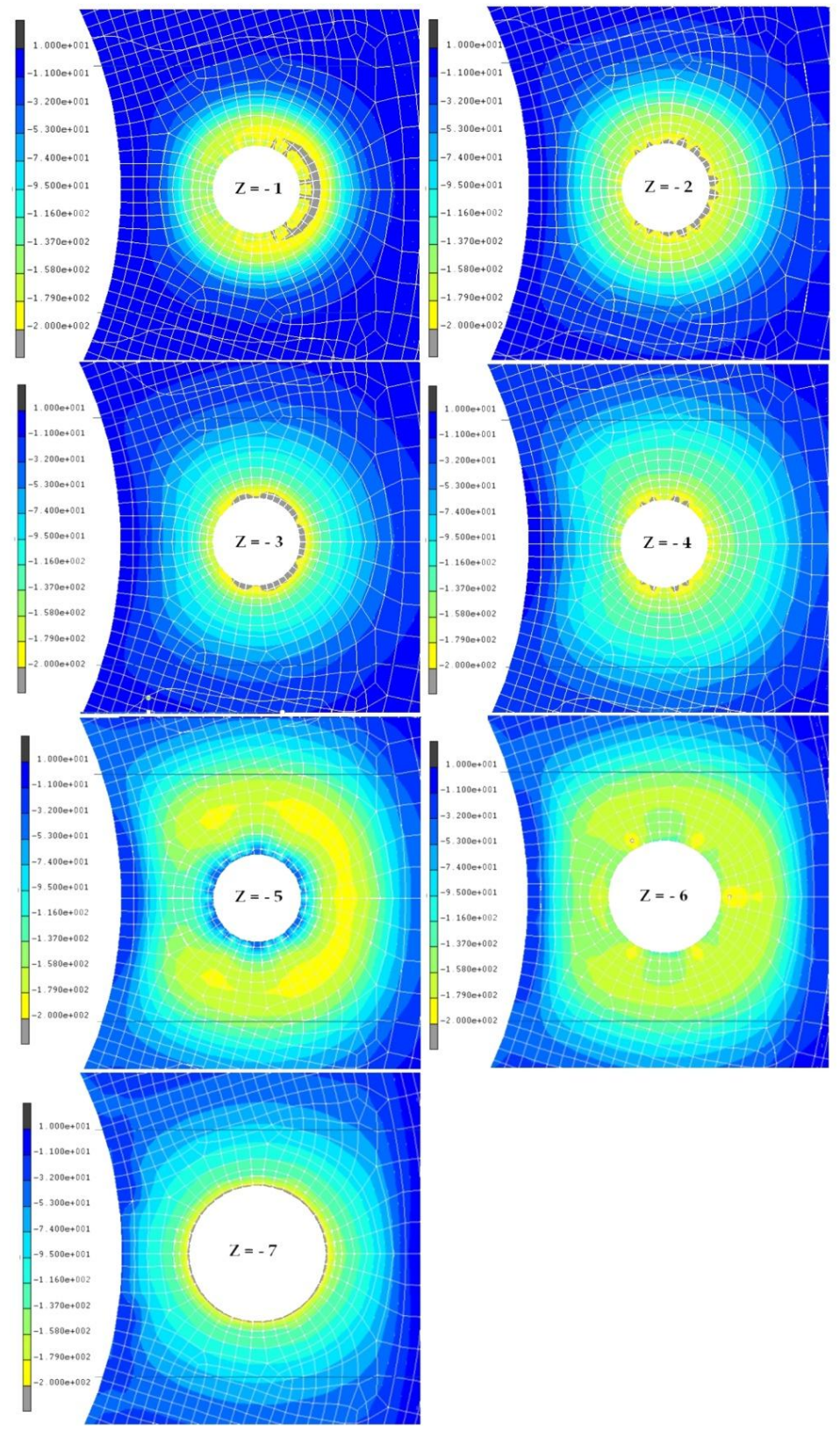

Figure 4.20: Minimum principal field in the flange. The measure of " $z$ " points out the distance of the section plane from the surface of contact with the flange. 
As shown in Figure 4.20, the flange is stressed in the radial direction (the direction assumed by the minimum principal stress in the surroundings of the hole) only in compression; the absence of traction stresses allows to exclude fatigue related issues in this component concerning radial stresses.
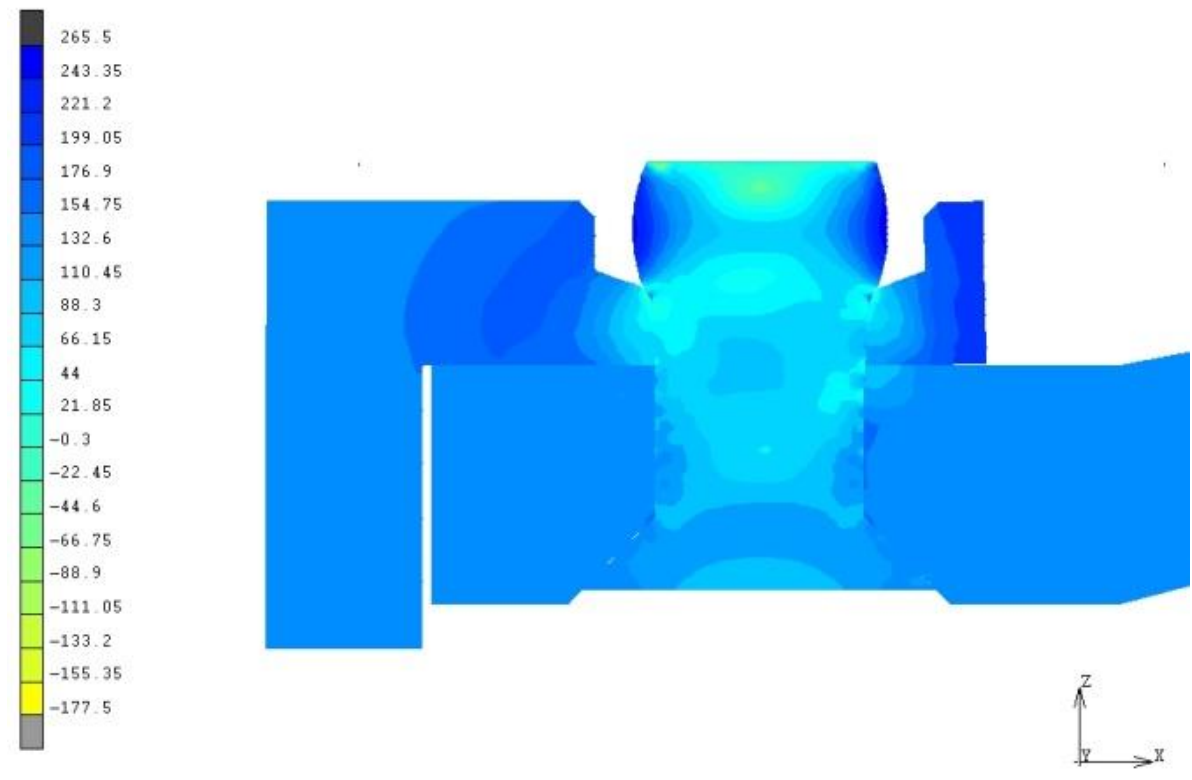

Figure 4.21: Maximum principal stress field

The maximum principal stress field assumes the circumferential direction in the surroundings of the hole (and the axial direction in the hole), and is the more dangerous for the fatigue strength of the assembly, since it reaches significant traction values in some regions. 


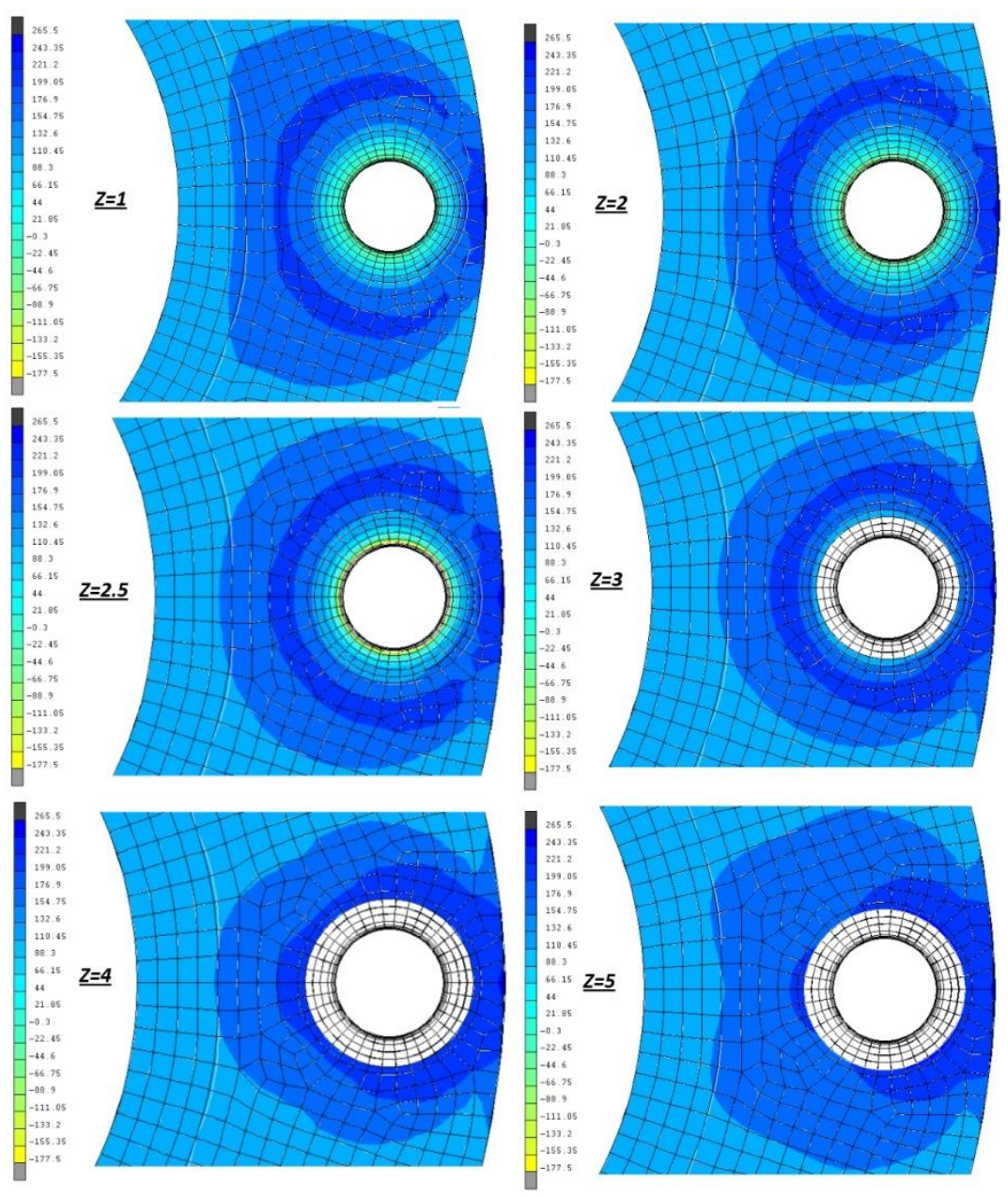

Figure 4.22: Maximum principal stress field in the shaft

Remembering that the circumferential direction is referred to the rivet axis, and therefore that this component of stress is significant only in the surroundings of the hole, we can notice in Figure 4.22 that the stress field reaches its positive maximum (i.e. the maximum traction stress value) on the outer surface of the shaft, reaching the value of $265 \mathrm{MPa}$. In that region the combination with the working condition could bring to crack initiation. Where the maximum principal stress becomes axial, its value is everywhere negative, so can be omitted in the fatigue analysis. 

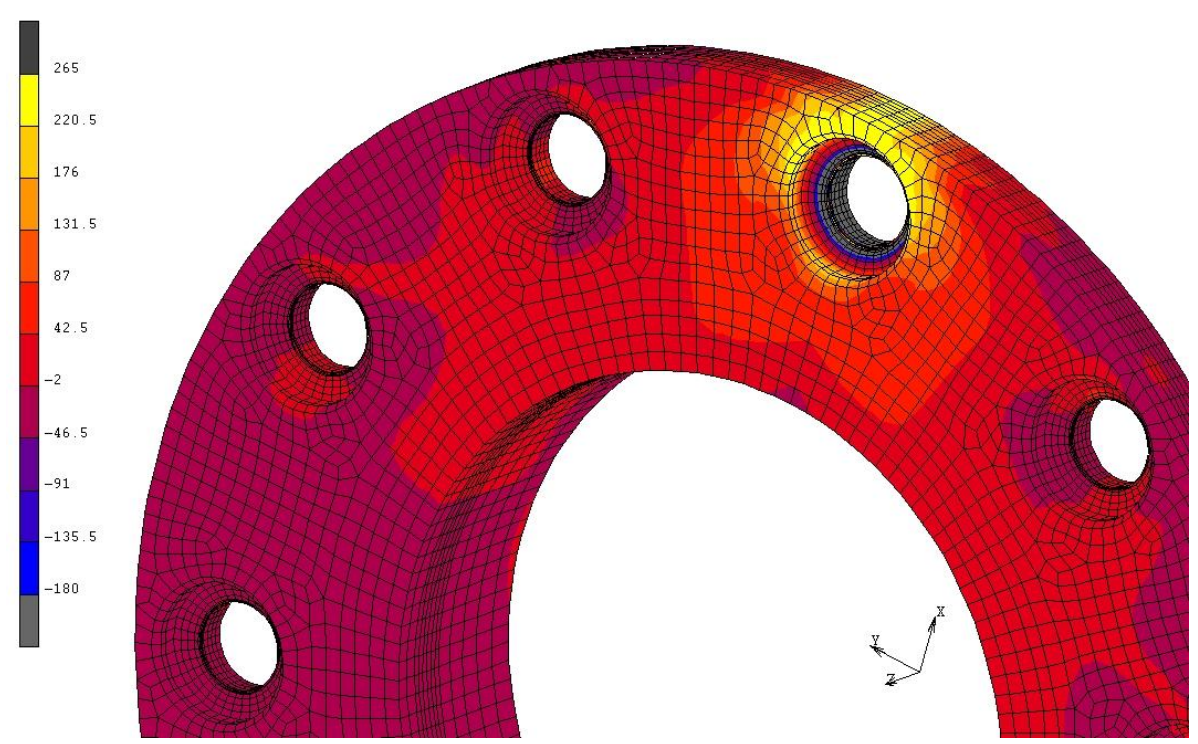

Figure 4.23: Maximum principal stress on the shaft

As far as it concerns the flange, in Figure 4.24 we can notice that, if the most diffused traction stress field is acting on the cylindrical surface adjacent to the rivet, there are some traction stresses also in the internal surface of the flange (with a maximum value around $75 \mathrm{MPa}$ ). We can suppose this is the most dangerous issue in the flange, because here even the working conditions (centrifugal force, bending moment due to the contact with the belt) develop traction stresses and, being a boundary surface, the surface roughness could be relatively high. From the past experience of the Piaggio Company, this is a critical part in the assembly: as shown in Figure 4.5, the material between the rivet and the internal surface of the flange is a typical region of crack initiation. 

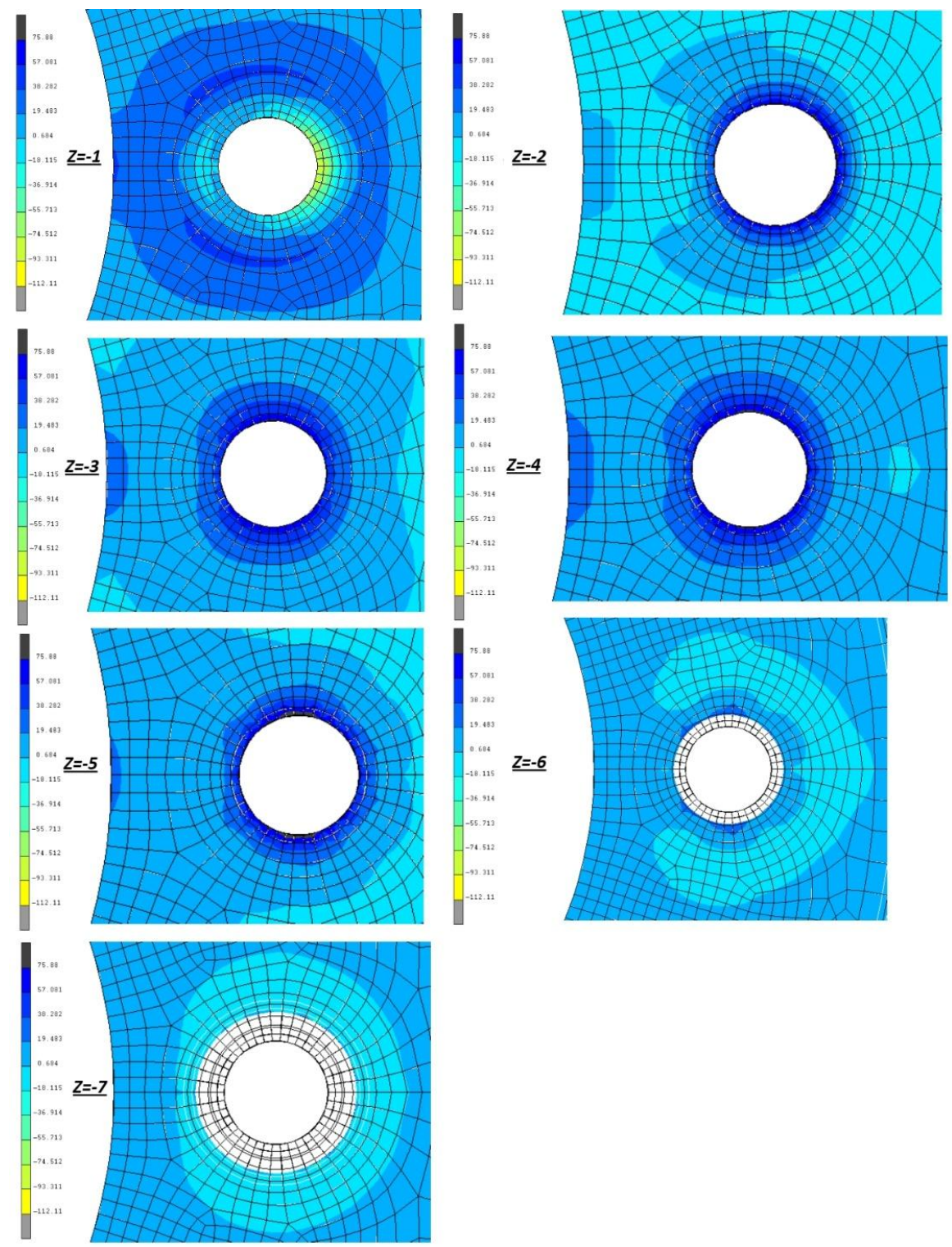

Figure 4.24: Maximum principal stress field in the flange

If the analysis of the minimum and the maximum principal components has helped to understand the direction of the stresses acting on the components of 
the assembly, to define the actual level of stress is useful to analyze the global equivalent stress, computed with the Von Mises formulation, reported in (4.21).

$$
\sigma_{e q}=\sqrt{\frac{1}{2}\left[\left(\sigma_{I}-\sigma_{I I}\right)^{2}+\left(\sigma_{I I}-\sigma_{I I I}\right)^{2}+\left(\sigma_{I I I}-\sigma_{I}\right)^{2}\right]}
$$

Where:

- $\sigma_{e q}$ is the Von Mises equivalent stress

- $\sigma_{I} \quad$ is the first principal component of the stress tensor

- $\sigma_{I I} \quad$ is the second principal component of the stress tensor

- $\sigma_{I I I}$ is the third principal component of the stress tensor.
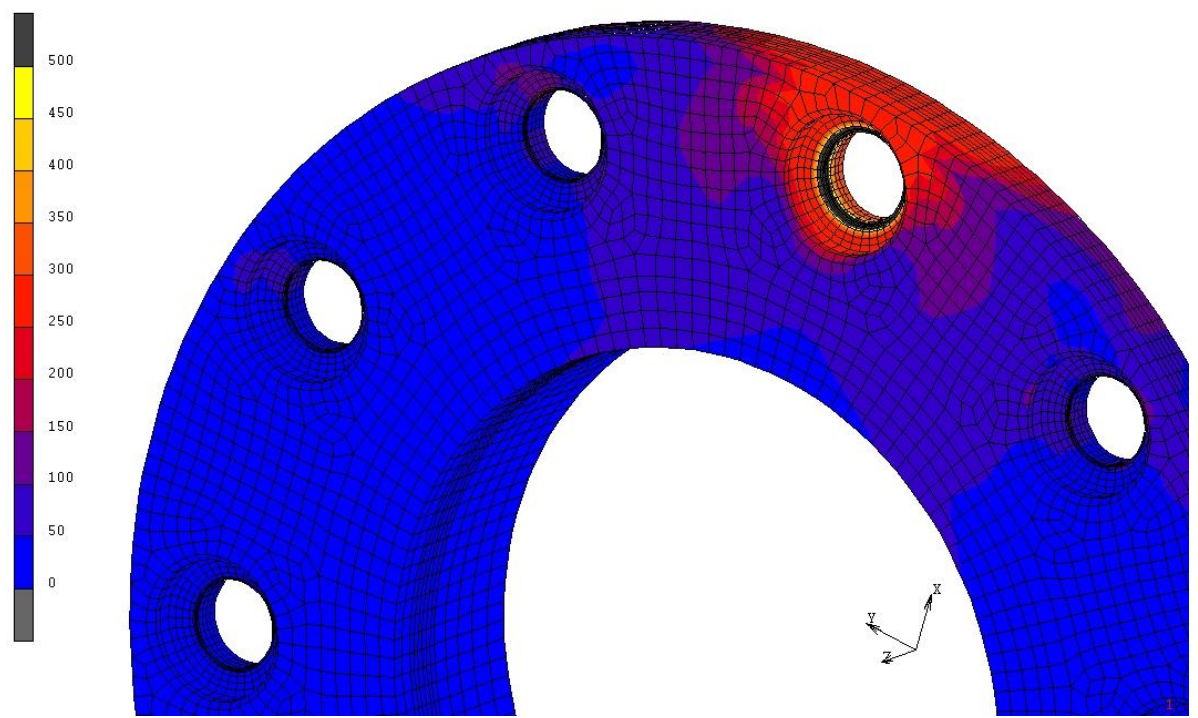

Figure 4.25: Von Mises stress field on the shaft

In Figure 4.25 is reported the Von Mises stress field acting on the shaft after the riveting process. The maximum value reached is equal to $450 \mathrm{MPa}$. The holes near the riveted one are interested in a stress field that reaches the maximum value of $100 \mathrm{MPa}$. This issue has to be considered in deciding the riveting order. 


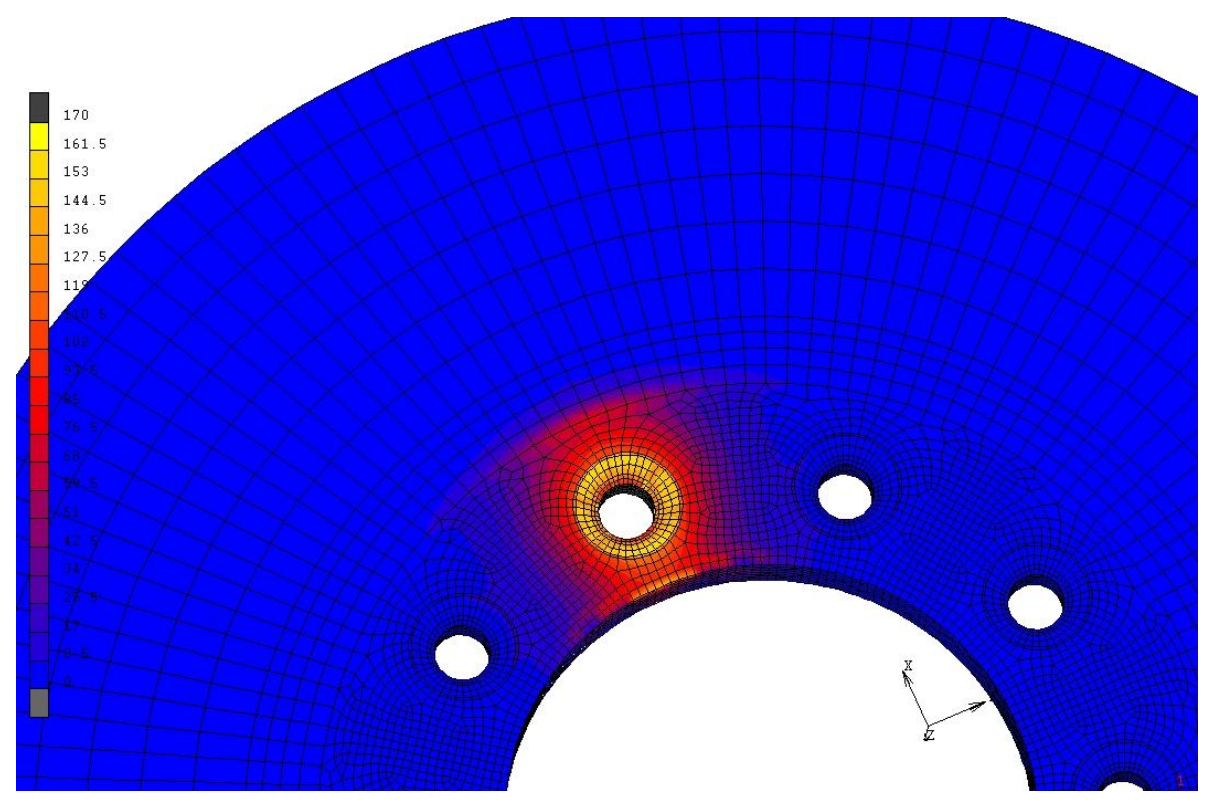

Figure 4.26: Von Mises stress field on the flange

In Figure 4.26 is reported the Von Mises stress field acting on the flange after the riveting process. The maximum value reached is equal to $165 \mathrm{MPa}$. The surrounding holes are not interested by the stress filed. 


\section{The fatigue strength of the flange material}

\subsection{Material and process matching}

In many cases, the material data used to proportionate the structural elements are measured in ideal conditions, with defect free specimens. This approach involves a saving in cost in the short period, because allows to obtain the material data with few experimental tests and to compile a general purpose database usable in many applications. Almost all the data available in literature are obtained with this method, so the characteristics of the "best conditions" material are generally easy to obtain. The most important limit of these data is the lack of correlation with the manufacturing process, on the other hand it has a great influence on the behavior of the material. For instance, a part realized with a forging process will show better mechanical characteristics (in terms of yield strength, ductility, toughness, life strength) if compared with the same part realized with a die cast process. In the better case, the production process can improve the behavior of the material, so this uncertainty could result in a global oversizing of the component. In the other cases, this uncertainty could be often resolved adopting high safety factors, thus adding mass to the designed components. However, in some cases the degradation of mechanic characteristic is so significant that even a reasonably high safety factor cannot avoid the failure of the part.

In this work, we have decided to measure the fatigue limit of the critical component of the pulley assembly: the flange, a component typically made by die cast of aluminum alloy. To match the material data with the manufacturing process, we have chosen to use a set of specimens milled from a die cast part, subdued to the same porosity level of the component in exam.

The investigated alloy is commonly called "Silafont 36" (commercial name of the AlSi9MgMn alloy), ad its composition is reported in the Table 5-1.

Table 5-1: "Silafont 36" composition, \% in mass

\begin{tabular}{|cccccccc|}
\hline Si & $\mathrm{Fe}$ & $\mathrm{Cu}$ & $\mathrm{Mn}$ & $\mathrm{Mg}$ & $\mathrm{Zn}$ & $\mathrm{Ti}$ \\
\hline $9.5 \div 11.5$ & 0.15 & 0.03 & $0.5 \div 0.8$ & $0.1 \div 0.5$ & 0.10 & $0.01 \div 0.15$ \\
\hline
\end{tabular}




\subsection{Testing approach}

Due to the low number of specimens available, we have chosen to execute the measure with a staircase approach [33]. This method is based on sequential tests executed with a prefixed number of cycles: the first specimen is tested with the fatigue strength expected for the material $\left(\sigma_{0}\right)$. If the failure occurs prior to the predefined number of cycles $\left(\mathrm{N}_{\mathrm{f}}\right)$, the second is tested at a lower level $\left(\sigma_{0}-d\right.$, where " $d$ " is the step between the stress levels). If the specimen reaches the imposed life (and is called "run out"), the second is tested with a higher level $\left(\sigma_{0}+d\right)$. If this procedure is carried out for a minimum number of 15 specimens, the stress level relative to a $50 \%$ probability of failure is computed with the equation (5.1).

$$
\sigma_{f}=\sigma_{0}+d \cdot\left[\frac{A}{N} \pm 0.5\right]
$$

The plus sign in the second term of the equation (5.1) is used if the less frequent event is the failure, and the minus sign if run outs are less frequent. The term " $N$ " is the number of the less frequent events, and " $A$ " is computed with the equation

$$
A=\sum i \cdot n_{i}
$$

Where:

- $\quad i \quad$ is the order of the stress level

- $n_{i} \quad$ is the number of occurrences of the less frequent event at the stress level $i$. 


\subsection{Results}

From the data available in literature, the expected fatigue strength for a die cast part made with the "Silafont 36" alloy is 90MPa. In Table 5-2 we have reported the results of the staircase fatigue test done on the 15 specimens available.

Table 5-2: The staircase table

\begin{tabular}{|c|c|c|c|c|c|c|c|c|c|c|c|c|c|c|c|c|c|c|c|c|c|}
\hline$\sigma$ & 1 & 2 & 3 & 4 & 5 & 6 & 7 & 8 & 9 & 10 & 11 & 12 & 13 & 14 & 15 & $\mathbf{x}$ & 0 & $\mathbf{i}$ & $\mathbf{n}_{\mathrm{i}}$ & $i^{*} n_{i}$ & $i^{2 t_{*}} n_{\mathrm{j}}$ \\
\hline 110 & & & & & & & & & & & $\bar{x}$ & & & & & 1 & 0 & 5 & 1 & 5 & 25 \\
\hline 100 & & & & & & & & $x$ & & 0 & & $x$ & & & & 2 & 1 & 4 & 2 & 8 & 32 \\
\hline 90 & $x$ & & & & & & 0 & & 0 & & & & $x$ & & $\mathrm{x}$ & 3 & 1 & 3 & 2 & 6 & 18 \\
\hline 80 & & $\mathrm{x}$ & & & & 0 & & & & & & & & 0 & & 1 & 2 & 2 & 1 & 2 & 4 \\
\hline 70 & & & $\mathrm{x}$ & & 0 & & & & & & & & & & & 1 & 1 & 1 & 1 & 1 & 1 \\
\hline 60 & & & & 0 & & & & & & & & & & & & 0 & 1 & 0 & 0 & 0 & 0 \\
\hline & & & & & & & & & & & & & & & & 8 & 6 & & 7 & 22 & 80 \\
\hline & & & & & & & & & & & & & & & & $\Sigma x$ & $\Sigma o$ & & $\mathbf{N}$ & A & B \\
\hline
\end{tabular}

Using these data, we can compute that the actual fatigue strength of the aluminum alloy is equal to $88 \mathrm{MPa}$, with a probability of $50 \%$. In Figure 5.1 we can see from the magnified view of the failing surface that the specimen material shows a great amount of porosity, that leads to a degradation of the strength of the alloy, and introduces a great scatter on its characteristics.
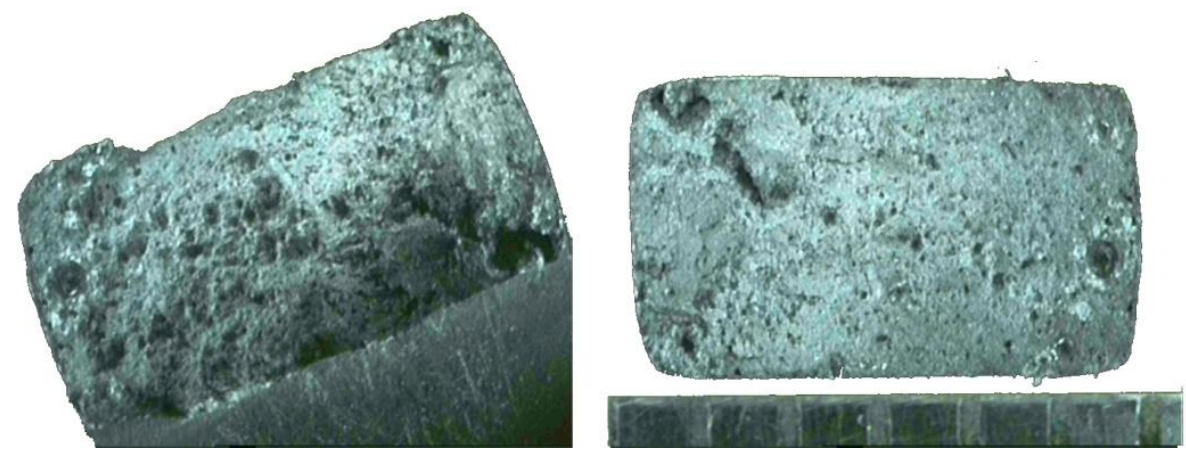

Figure 5.1: Magnified view of the failing surface of the specimen 
In detail, the standard deviation of the measured value can be computed using the equation (5.3).

$$
\hat{\sigma}=1.62 \cdot d \cdot\left[\frac{N B-A^{2}}{N^{2}}+0.029\right]
$$

Where

$$
B=\sum i^{2} \cdot n_{i}
$$

Using the data reported in Table 5-2, the standard deviation is $26 \mathrm{Mpa}$. In Figure 5.2 we have reported the failure probability diagram for the flange material. Using this graph it is possible to define the reliability of the material under a given level of alternate stress.

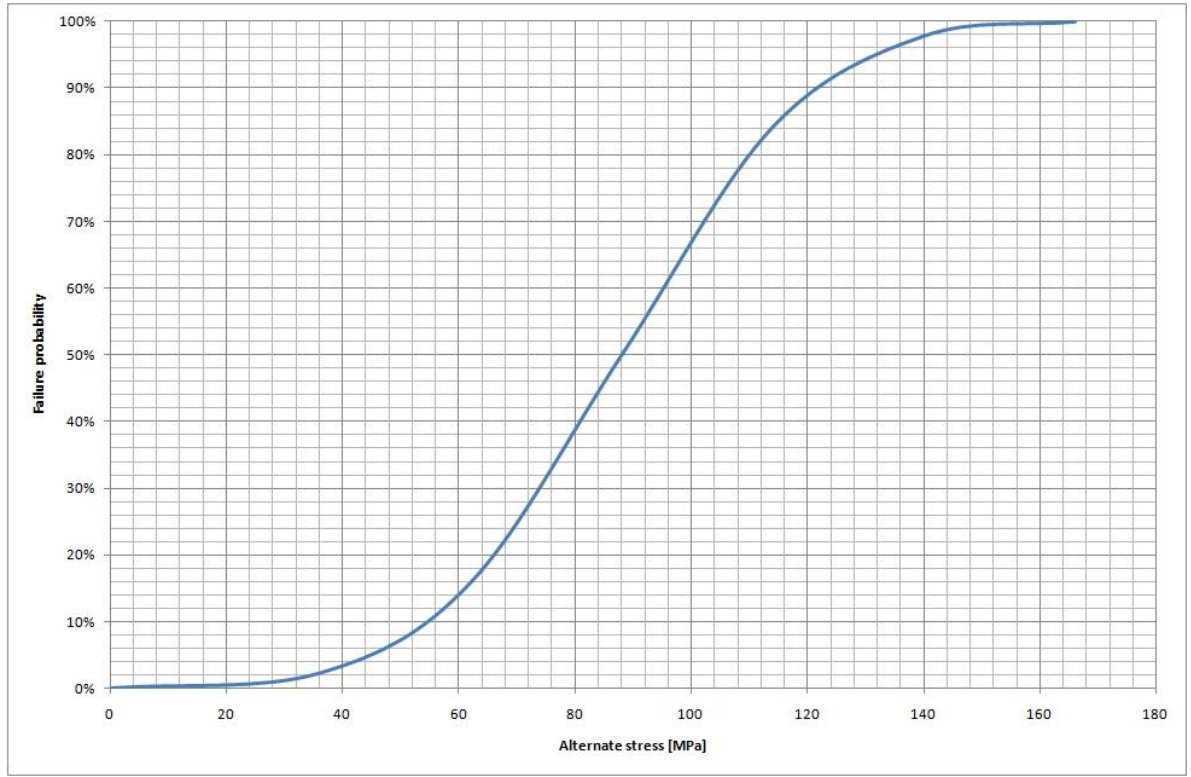

Figure 5.2: Failure probability of the flange material 


\section{The stress field acting on the pulleys in working conditions}

\subsection{The fatigue calculation approach}

In order to carry out the fatigue calculations based on the infinite life conditions, we have reduced the multi axial stress field to uniaxial one by means of the Von Mises criteria, using the Goodman correction for the mean stress effect.

We have derived the Haigh fatigue curve from the data acquired with the experimental tests previously exposed, so the curve is affected by some approximations. As prescribed by the Goodman correction, the Haigh curve (reported in Figure 6.1) has been obtained with a linear relation between the alternate stress and the mean stress, imposing the fatigue limit for a pure alternate loading and the ultimate strength for a pure static loading.

According to the approach proposed by Von Mises and modified by Juvinall [34], we have used the Haigh curve to define the equivalent alternate stresses acting on the flange, considering the mean stresses equal to the positive values assumed by the maximum principal stress in the riveting process. The negative values of the maximum principal stress are omitted because of to their positive effect on the fatigue strength of the material. 


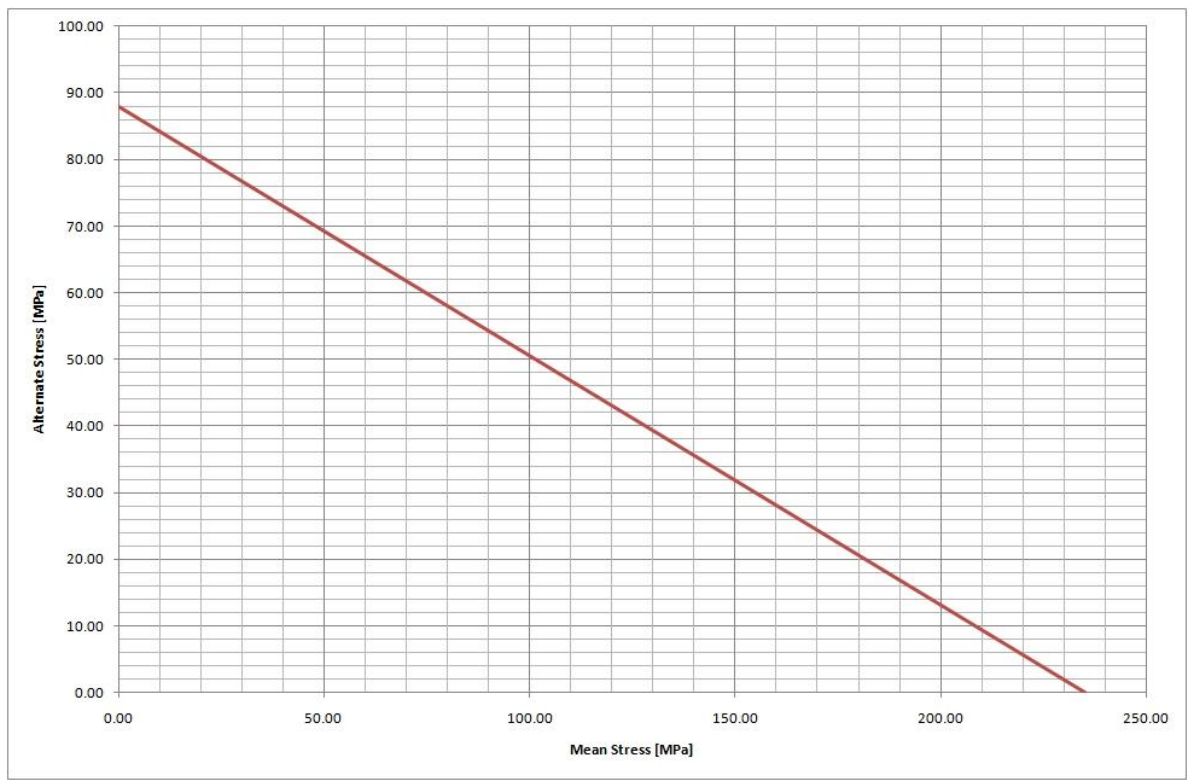

Figure 6.1: The Haigh curve of the flange material

Since the mean stress is due to the residual stress field generated by the riveting process, we have computed the equivalent alternate stresses with the equation (6.3). It imposes the same safety factor between the actual load case and the equivalent pure alternate load case under the assumption that the mean stress remains constant while the alternate stress changes.

$$
\sigma_{a_{e q}}=\sigma_{a} \frac{\sigma_{u}}{\sigma_{u}-\sigma_{m}}
$$

Where:

- $\sigma_{a_{e q}}$ is the equivalent alternate stress

- $\sigma_{a} \quad$ is the measured alternate stress

- $\sigma_{m}$ is the measured mean stress

- $\sigma_{u} \quad$ is the ultimate strength of the material. 
In Figure 6.2 we have reported a graphical explanation of the meaning of the equation (6.3): as shown in the equation (6.4), the ratio between the fatigue strength of the material and the equivalent stress is equal to the ratio between the actual stress and the failure condition considering an increase in alternate stress and the mean stress as a constant of the stress field.

$$
\frac{\sigma_{f}}{\sigma_{e}}=\frac{\sigma_{\max }}{\sigma_{m}}
$$

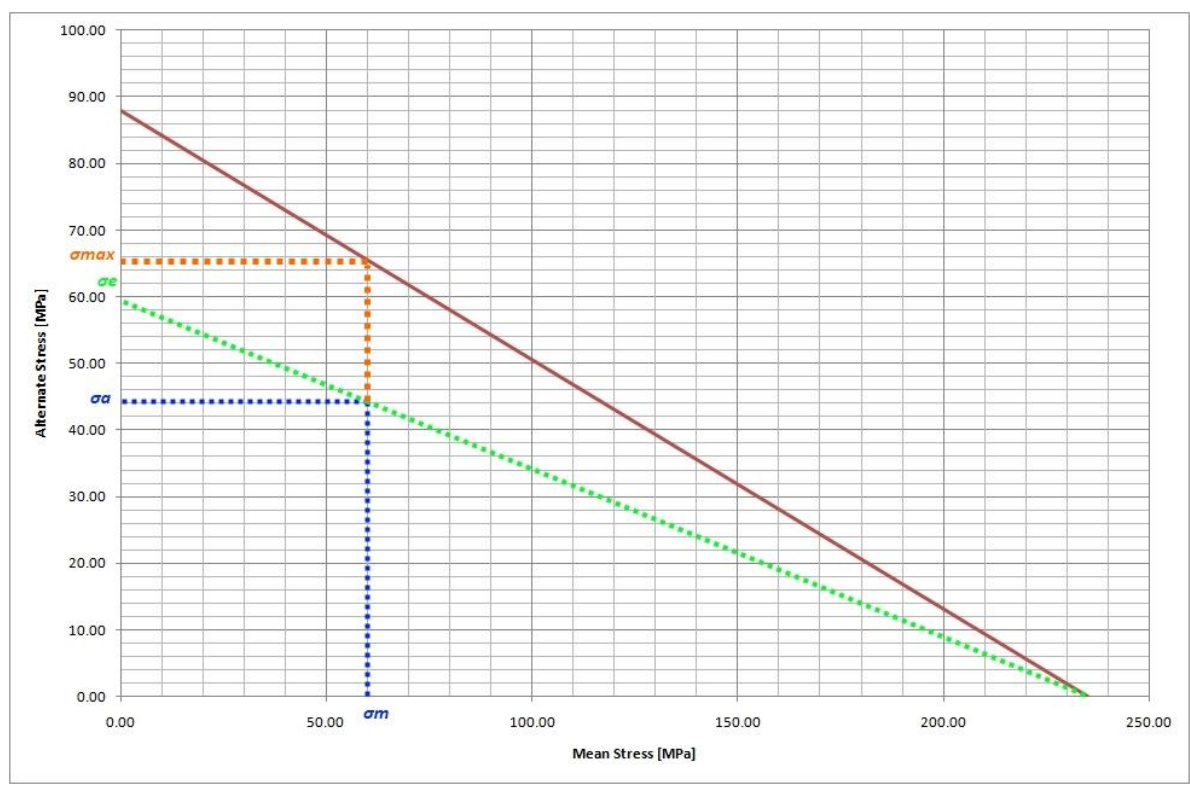

Figure 6.2: Graphical meaning of the equation (6.3) 


\subsection{The FE analysis of the working conditions}

\subsubsection{The CAD model}

To evaluate the stress field acting on the flange under the working conditions, we have used the contact force measured with the MultiBody model described in chapter 1 . To apply the contact force, we have used a series of belt elements (with the same length of the elements used in the MB analysis) disposed on the surface of the flange, at a distance from the axis equal to the pitch diameter for a given load case. The number of belt elements is clearly defined by the actual wrapping angle of the belt (see Figure 6.3). The use of a "discretized belt" on the CAD model has allowed to apply the contact force with the same accuracy of the $M B$ analysis. As shown in Figure 6.4, we have included the rivets in the CAD model, in order to evaluate correctly the reaction forces acting on the flange holes. Since the shaft was not interesting in this analysis, we have chosen to simplify the geometry of the rivet on the punched side.

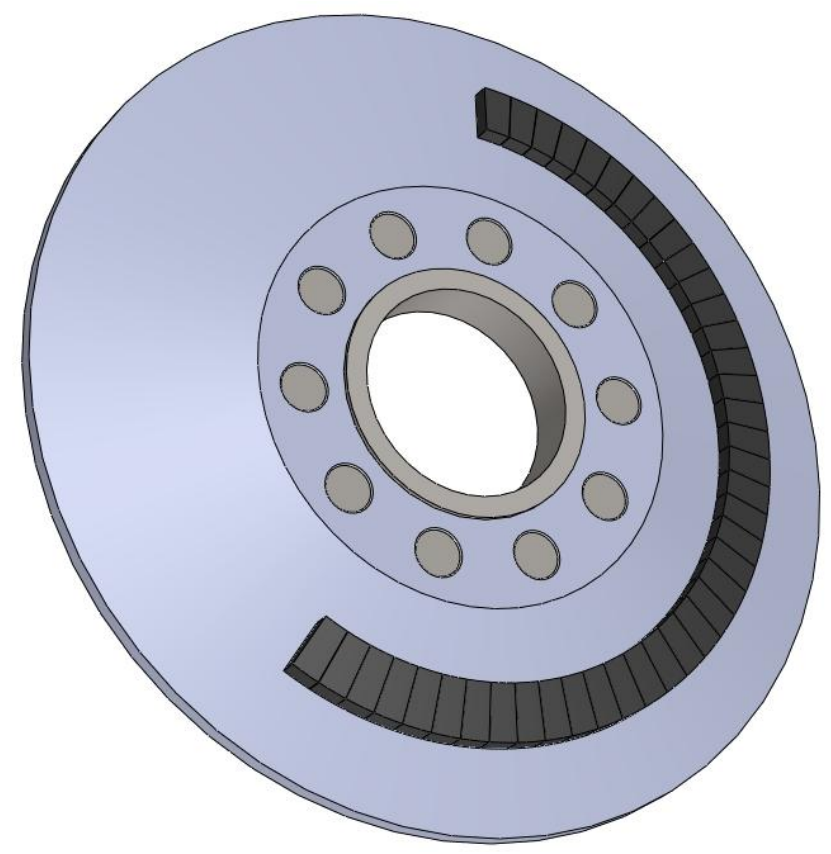

Figure 6.3: The CAD scheme of the working model 


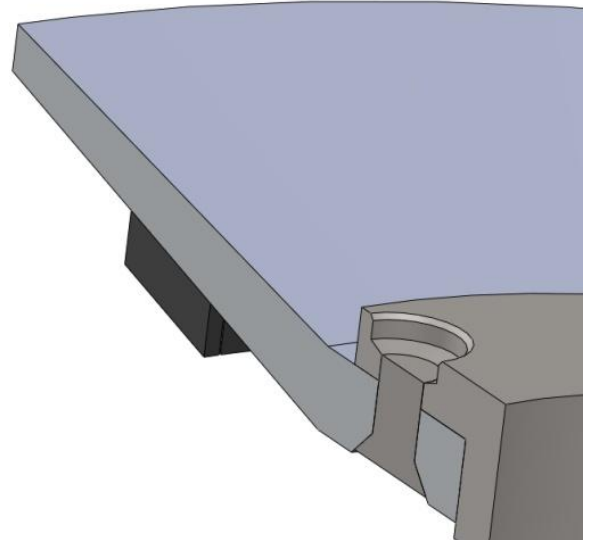

Figure 6.4: The rivet 


\subsubsection{The FE model}

The CAD model has been meshed with 66900 nodes and 29800 elements. In Figure 6.5 the result of the mesh operation are reported. The same meshed model has been used for each load case, in order to simplify the fatigue calculation, avoiding the loss of references for the nodes.

We have modeled with the same approach discussed in chapter 4.4 .3 the interfaces between the rivets and the flange and between the flange and the shaft. Since, as stated before, the shaft behavior is not interesting in this analysis, we have modeled the contact between the rivets and the shaft as a bonded surface, in order to keep low the computational cost of the simulation. Moreover, since the forces acting on the belt elements are purely compressive, we have modeled as bonded also the interface between the belt elements and the pulley. Since the loads used were obtained from the MB model, we have excluded every connection between the belt elements, ensuring the correct load application.

As shown in Figure 6.6, we have applied the measured contact force on each belt element; moreover, we have introduced the rotational velocity measured with the $\mathrm{MB}$ analysis, in order to consider the forces related to the centrifugal acceleration. The joint with the ground has been imposed to the internal surface of the shaft. This constraint is consistent with the real world, and does not affect the solution on the flange nodes.

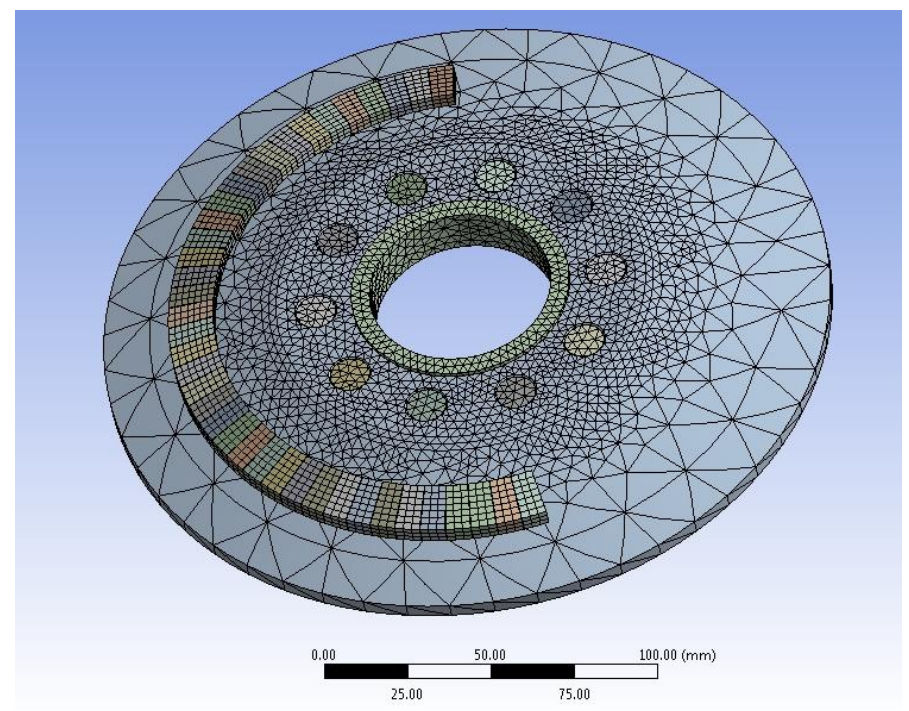

Figure 6.5: The meshed model 


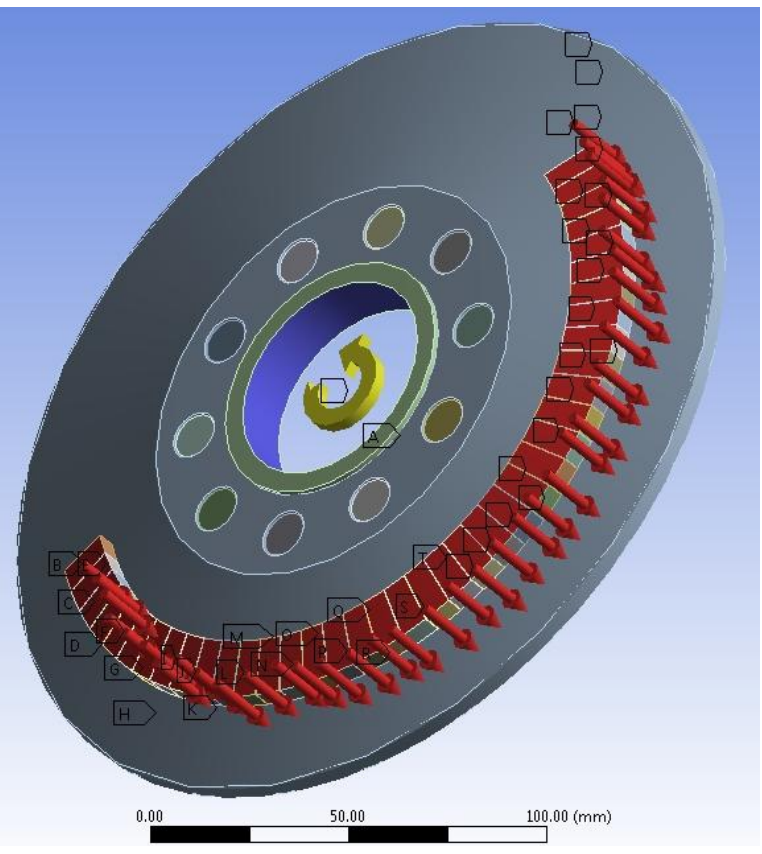

Figure 6.6: Load application 


\subsubsection{The first load case: the riveted flange}

Once computed the stress field acting on the pulley after a single riveting process, we have to apply the results of the analysis to the other holes, in order to define the residual stress field acting on the flange after the punching of ten rivets. Since the simulation of a full riveting process would not have converged, we have applied on the surfaces of the shaft and of the flange the reaction forces measured with the analysis exposed in the chapter 1.

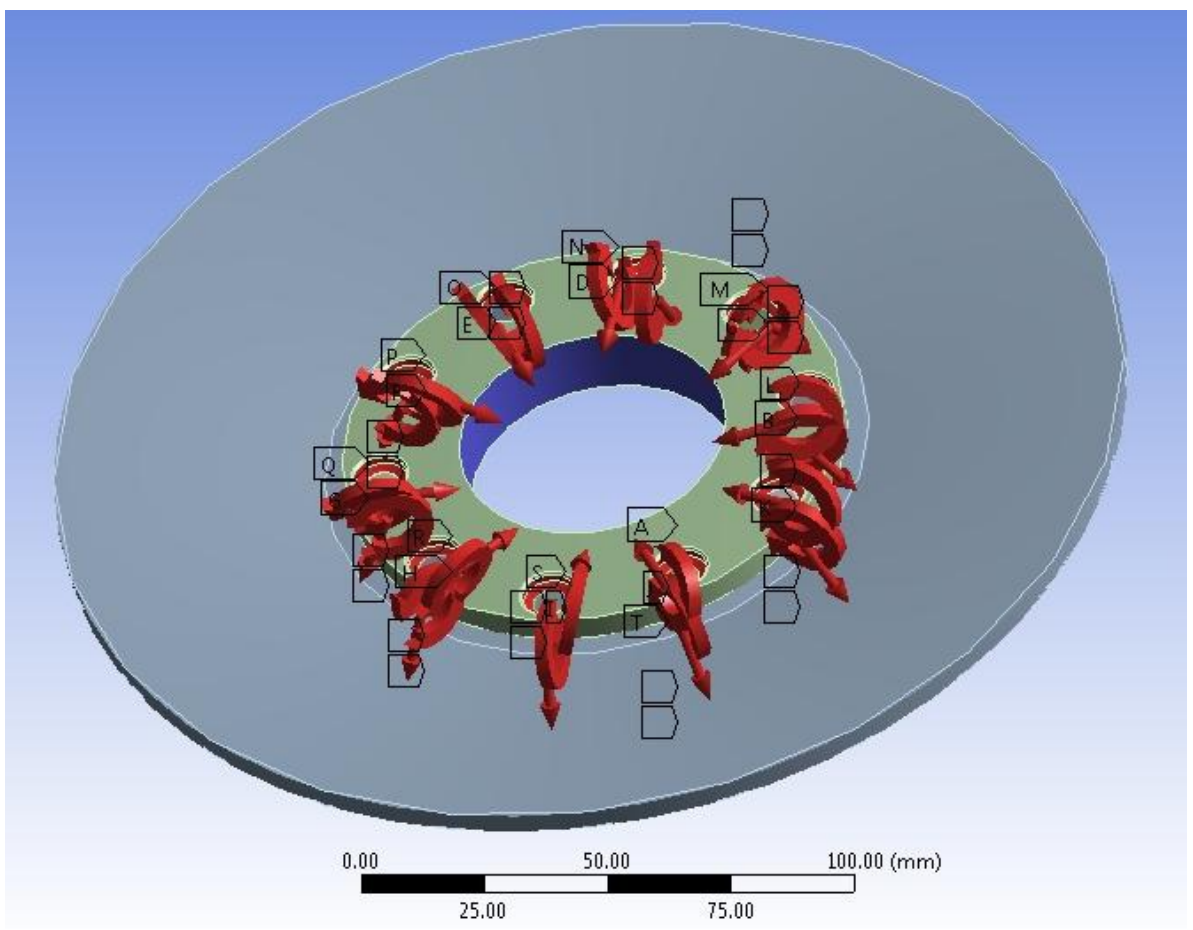

Figure 6.7: The full riveting residual stress

To obtain the actual stress field, we have applied the history of reaction forces measured: applying only the final reaction force would not have allowed to consider the effects due to the work hardening of the materials involved in the process. According to the considerations exposed in 6.1, the most important output of this simulation is the maximum principal stress field, that has been used to compute the equivalent alternate stress acting on the flange. 


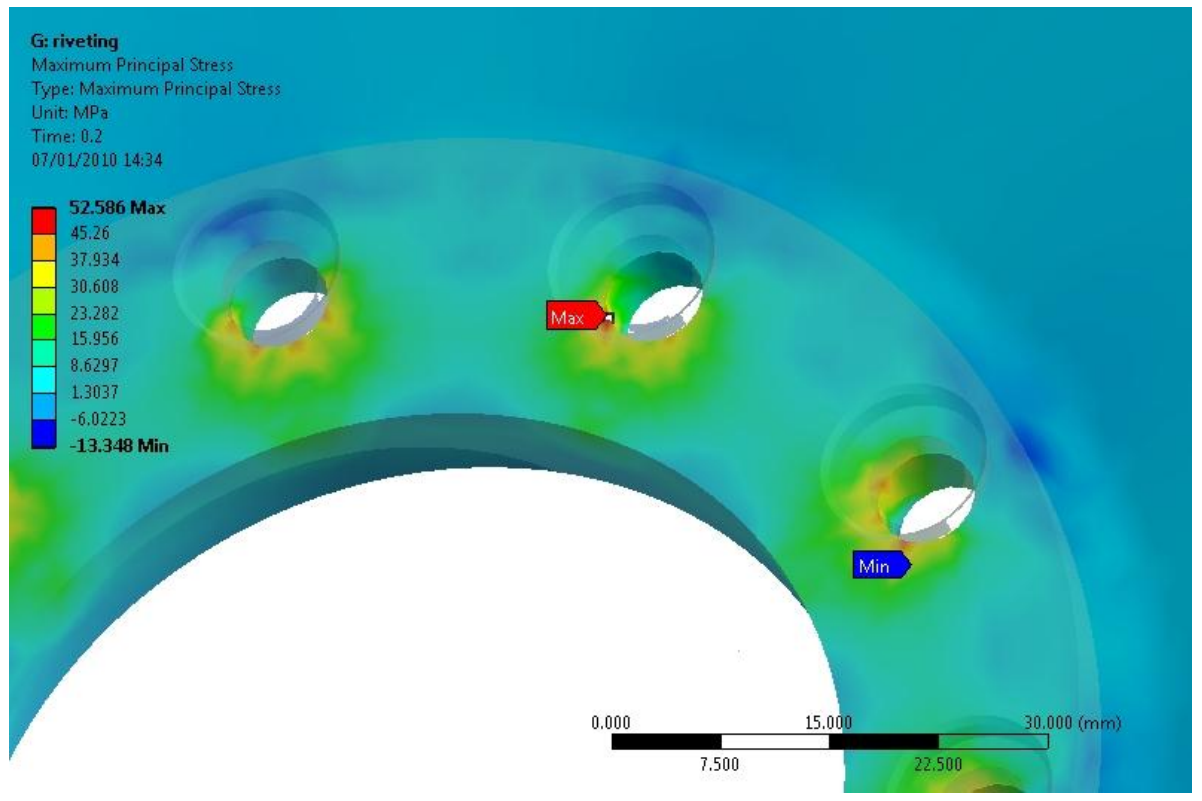

Figure 6.8: Maximum principal stress field on the flange 


\subsubsection{The maximum torque conditions}

Using the results of the analysis exposed in the chapter 3.4.1, we have plotted the contact force measured in maximum torque conditions as a function of the wrapping angle of the belt on the pulley; then, as shown in Figure 6.9, we have sampled the measured data in angular step equal to the angular dimension of the belt, assuming for each sample the maximum value of the contact force measured within the sampling range. This operation has led to a set of 41 values of contact force, that have been applied to the belt elements bonded to the flange, as shown in Figure 6.9. This set of loads generates a resultant force equal to $2035 \mathrm{~N}$, applied $35.5 \mathrm{~mm}$ away from the axis.

In Figure 6.10 is reported the Von Mises stress field resultant from the action of the contact force and the centrifugal acceleration.

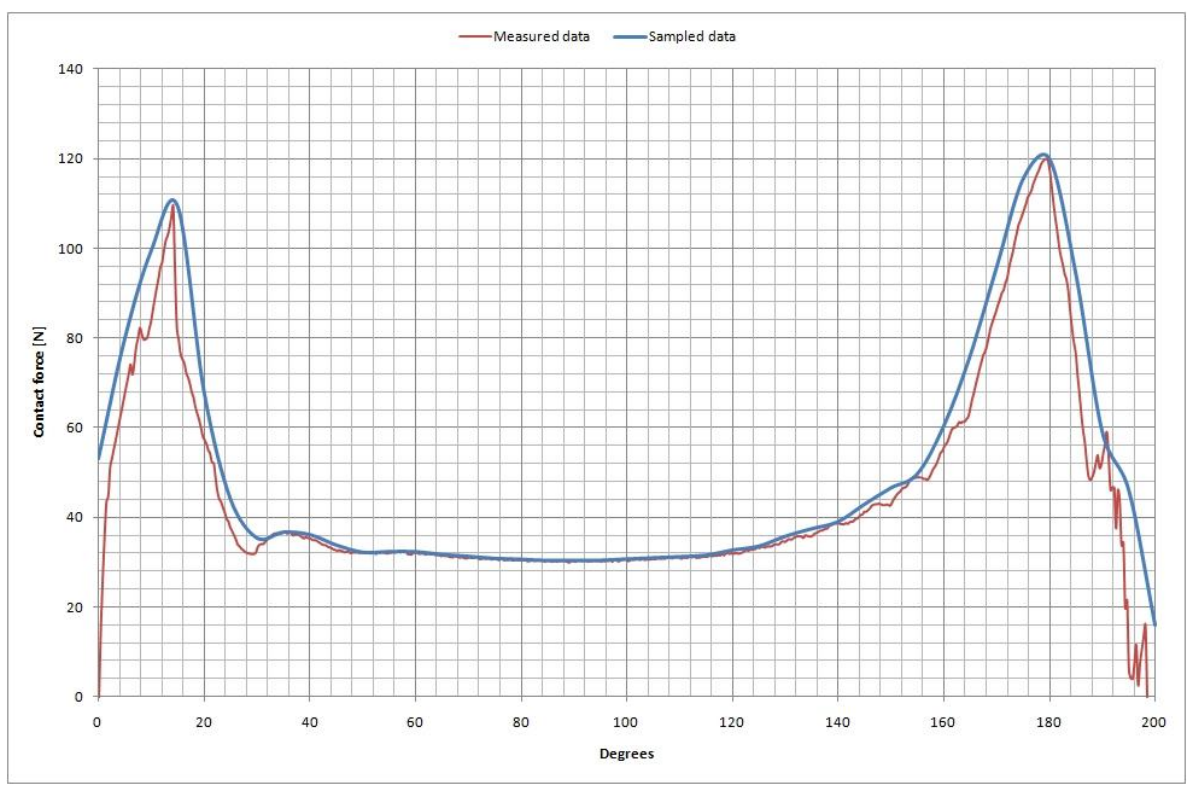

Figure 6.9: Contact force in maximum torque conditions 


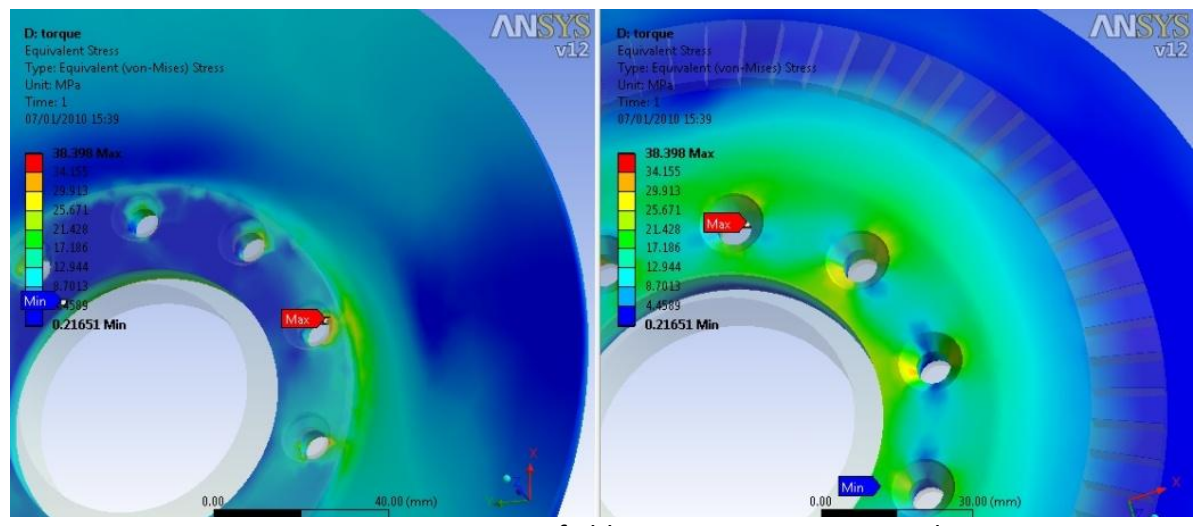

Figure 6.10: Von Mises stress field in maximum torque conditions

The obtained Von Mises stress field has been exported in a spreadsheet in order to compute the equivalent alternate stress on each node with the equation (6.3). The maximum value reached by the equivalent alternate stress is $39 \mathrm{MPa}$, as shown in Figure 6.11; using this datum in the failure probability curve we can compute that the reliability of the flange under this load case is equal to $97 \%$.

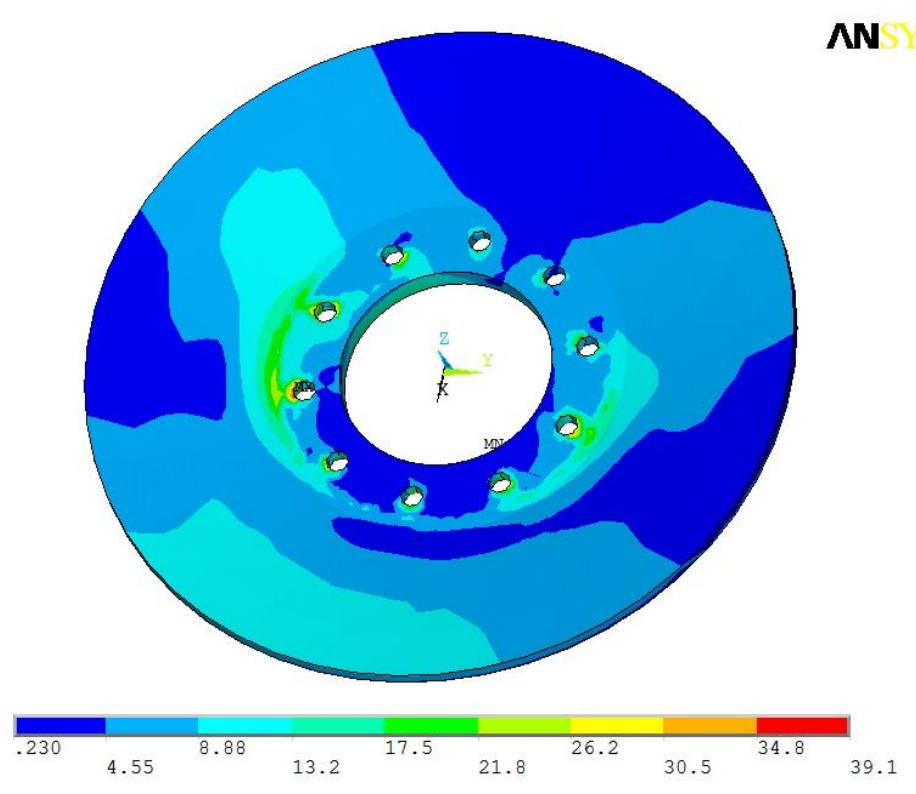

Figure 6.11: Equivalent alternate stress field in the maximum torque conditions 


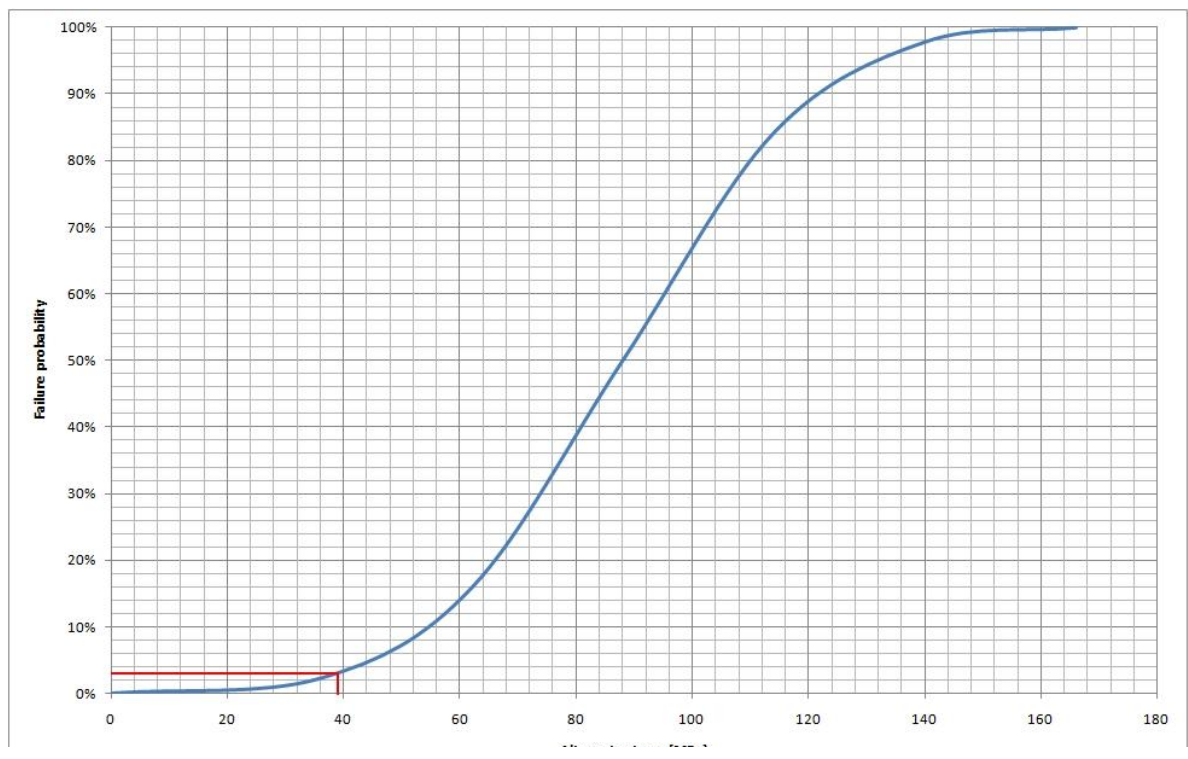

Figure 6.12: Probability of failure in maximum torque conditions 


\subsubsection{The maximum power conditions}

With the same approach used in the previous chapter, we have evaluated the contact force field acting on the pulley in maximum power conditions. The contact force is reported in Figure 6.13 as a function of the wrapping angle of the belt on the pulley.

The maximum value reached by the equivalent alternate stress is $54 \mathrm{MPa}$, as shown in Figure 6.15; using this datum in the failure probability curve we can compute that the reliability of the flange under this load case is equal to $90 \%$.

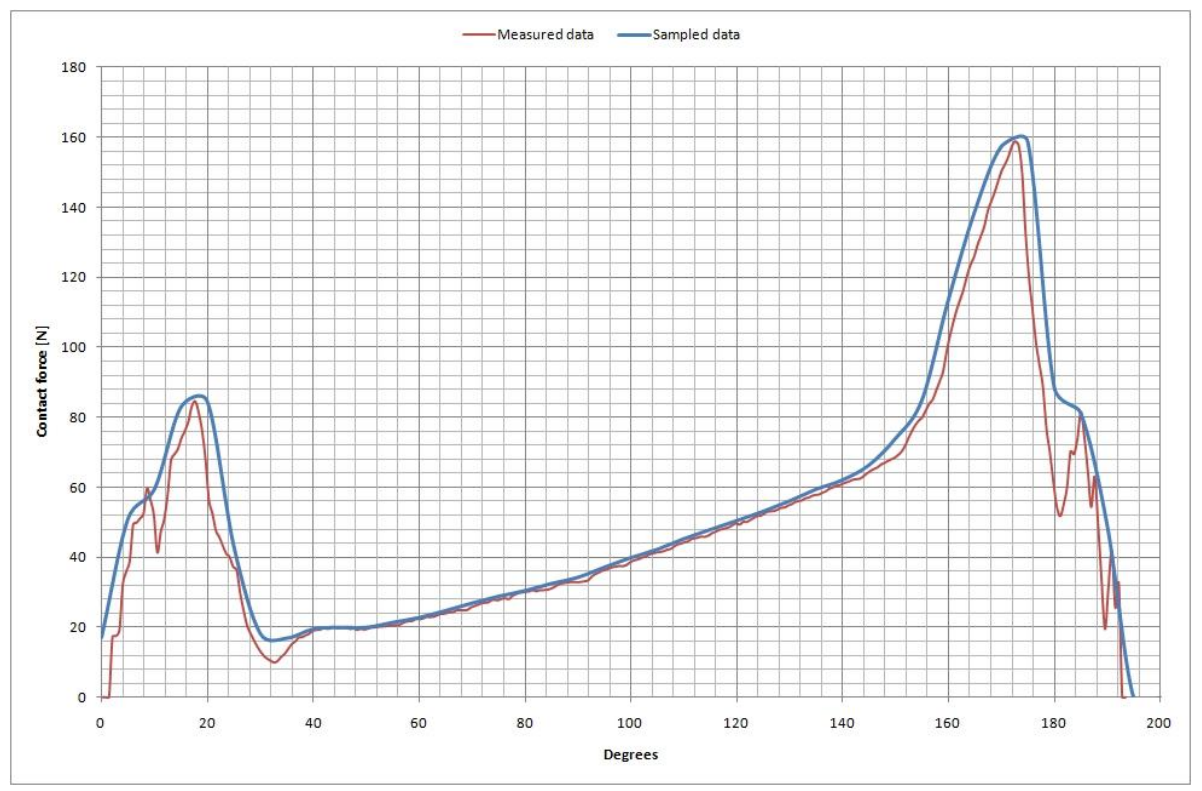

Figure 6.13: Contact force in maximum power conditions 


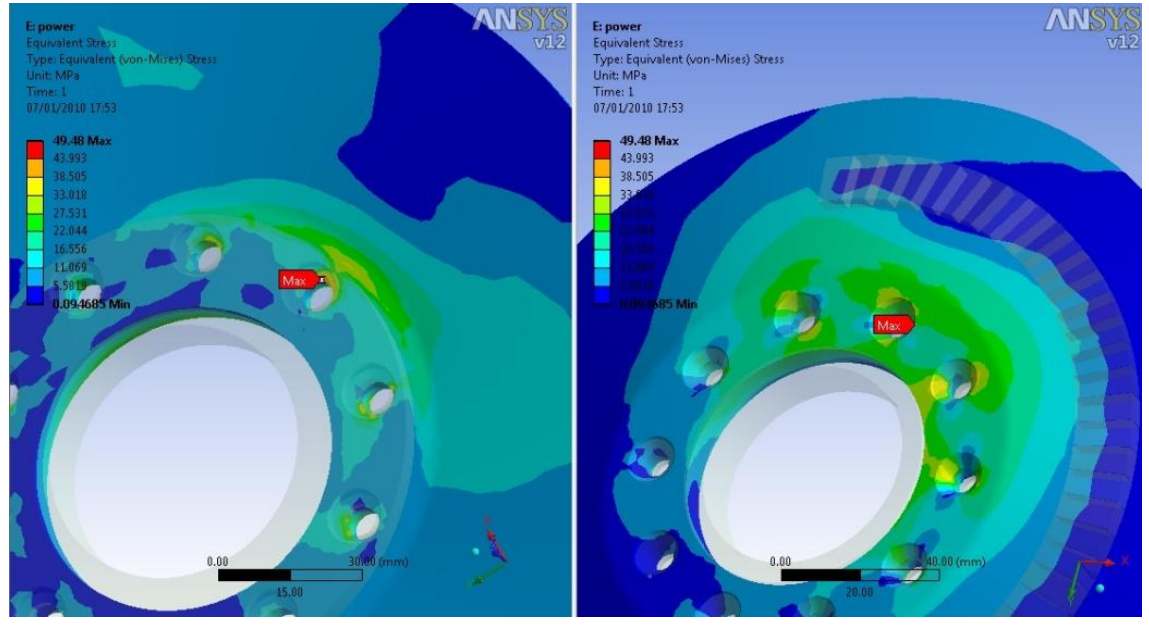

Figure 6.14: Von Mises stress field in maximum power conditions

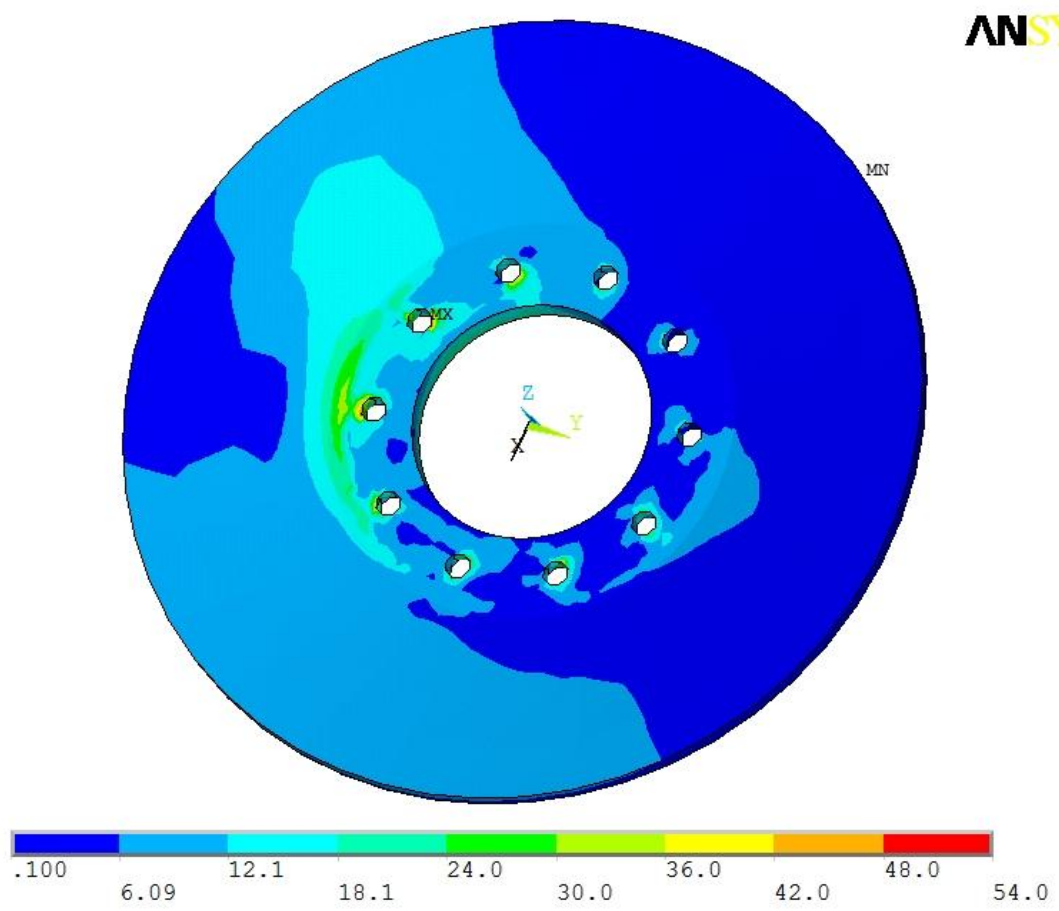

Figure 6.15: Equivalent alternate stress field in the maximum power conditions 


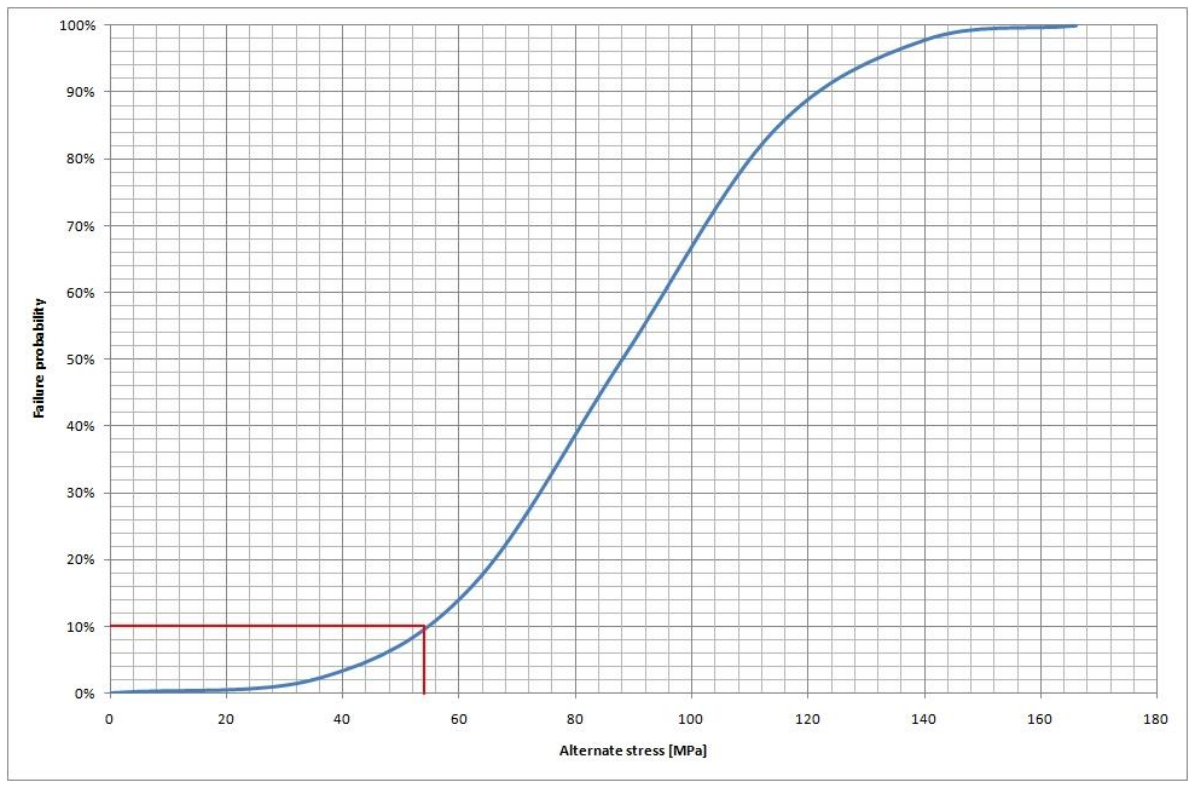

Figure 6.16: Probability of failure in maximum power conditions 


\subsubsection{The over speed conditions}

With the same approach, in Figure 6.17 is reported the contact force as a function of the wrapping angle. The resultant force is equal to $2590 \mathrm{~N}$, applied $50.3 \mathrm{~mm}$ away from the axis.

Even in this load case the equivalent alternate stress is lower than the fatigue strength of the material: the Von Mises stress field, reported in Figure 6.18, generates a maximum equivalent tension of $70.5 \mathrm{MPa}$ if combined with the residual stress field, as shown in Figure 6.19. Using this datum in the failure probability curve we can compute that the reliability of the flange under this load case is equal to $75 \%$.

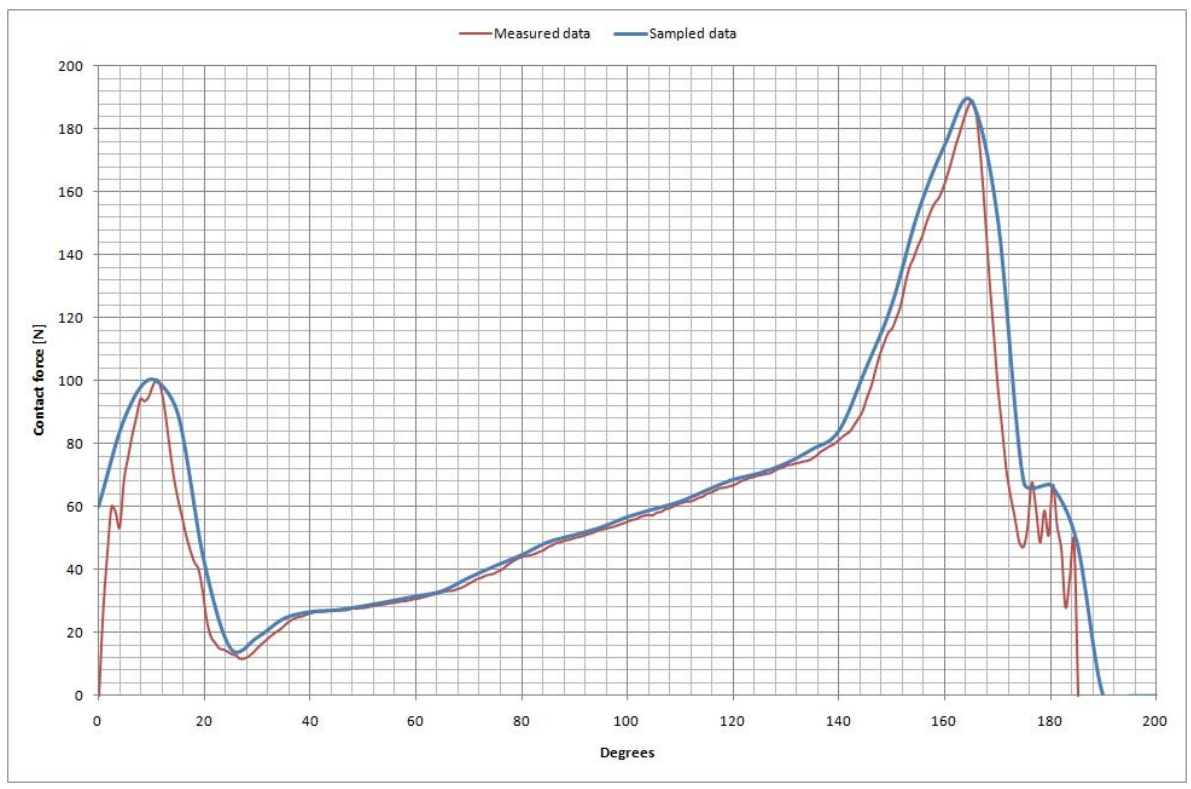

Figure 6.17: Contact force in over speed conditions 


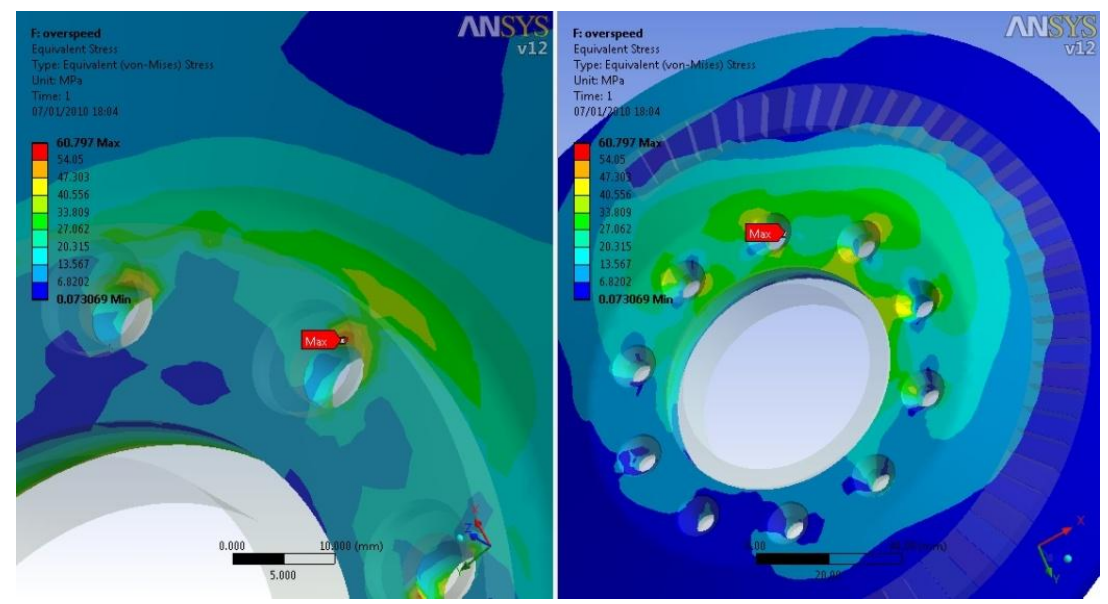

Figure 6.18: Von Mises stress field in over speed conditions

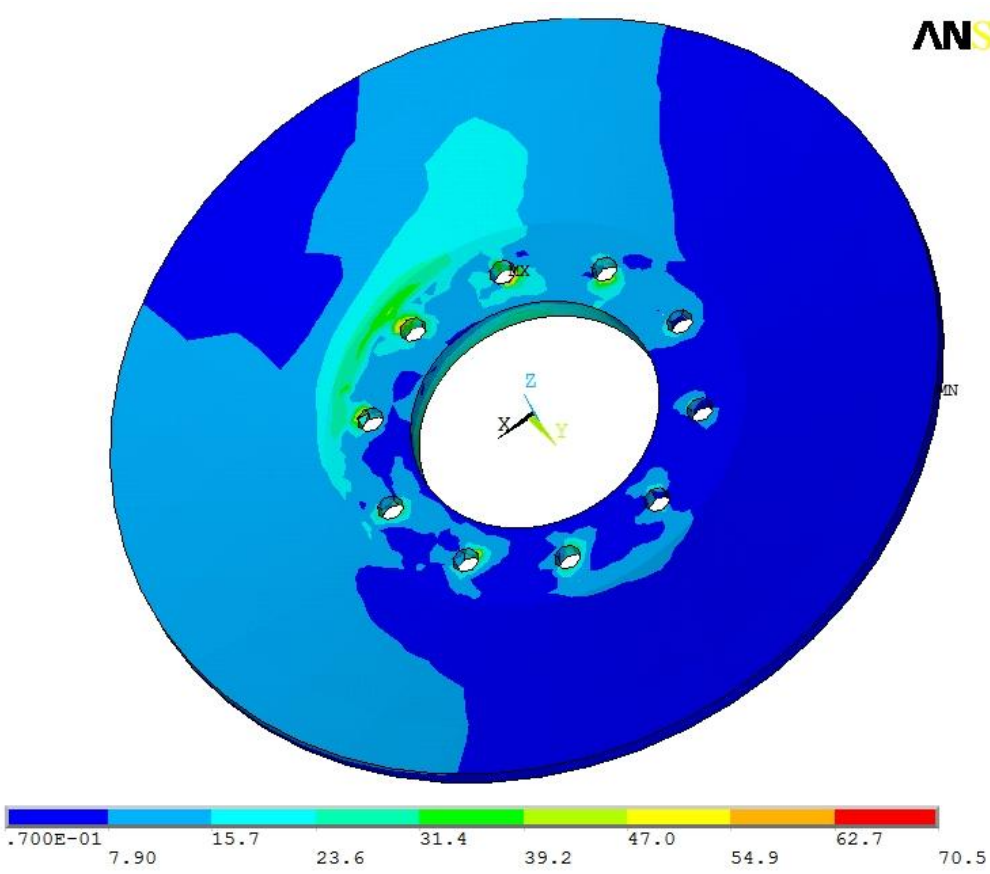

Figure 6.19: Equivalent stress field in the over speed conditions 


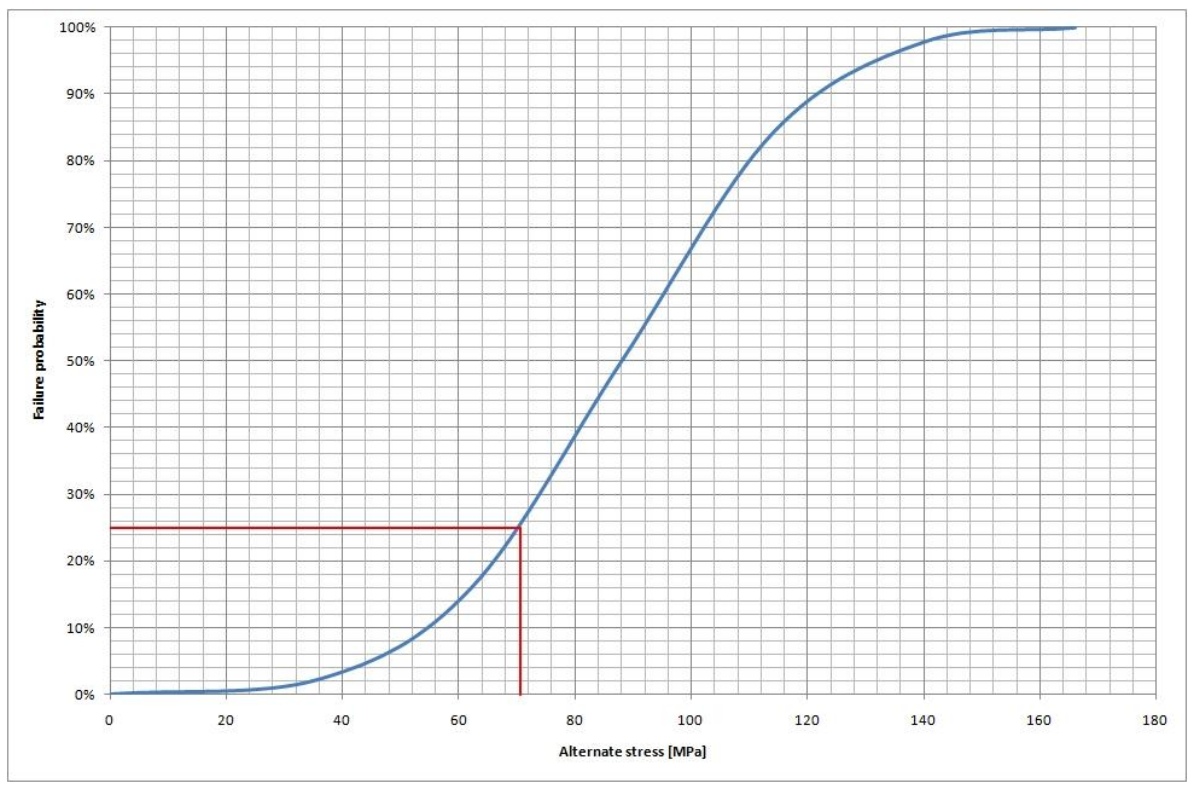

Figure 6.20: Probability of failure in over speed conditions 


\subsection{The reliability of the flange}

Considering an infinite lifetime expectation, the standard Piaggio test at the engine bench can be thought as a series of infinite lifetime tests on the flange, executed with the three load cases.

Under these conditions, the failure of the flange could occur in each load case. We can therefore affirm that the probability of survival of the flange to an infinite test at the engine bench is equal to the worst reliability among the ones computed for each load case. Assuming the reliability of the flange as the reliability of the material for a given alternate stress level, we can report in Table 6-1 the reliability computed for each load case.

Table 6-1: Reliability of the flange

\begin{tabular}{|c|c|}
\hline Load Case & Reliability \\
\hline Maximum torque & $97 \%$ \\
\hline Maximum power & $90 \%$ \\
\hline Over speed & $75 \%$ \\
\hline
\end{tabular}

Since over speed is the critical condition, we can affirm that the global reliability of the flange is equal to the one computed in this load case (75\%). 



\section{Conclusions}

In this work we have analyzed the stress field acting on the critical components of the Continuously Variable Transmission (CVT) mechanism used on the high displacement scooters produced by the Piaggio Company. Focusing on the behavior of the belt and the driven flange, we have analyzed the stresses acting on these components, considering the residual stress field due to the riveting of the flange on the output shaft and the alternate stresses due to the working conditions. To achieve these results, we have realized a full MultiBody (MB) model of the CVT, including all the degrees of freedom of the mechanism to avoid the approximations introduced in other works. The belt has been modeled with a Finite Element (FE) approach, discretizing its whole structure and assigning the correct material characteristics to the various components (Kevlar plies, elastomeric cogs, etc.). This kind of analysis has led to an accurate schematization of the mechanism, that has given important results on the level of stress of the various parts of the CVT.

The load history computed with the MB model has been used to define the estimated lifetime of the belt, using the proportioning system supplied by the belt producer with the Miner approach. This analysis has shown that the estimated lifetime of the belt under the Piaggio standard test at the engine bench is lower than 70 hours.

The forces measured in the contact between the belt and the pulleys have been used to define the alternate stress acting on the flange, while the residual stress field has been computed with a three dimensional non linear analysis of the riveting process. We have taken into account the work hardening of the materials involved in the process; in detail, the flange material behavior has been characterized by an experimental traction test.

Since the flange is made with a die cast process, we have chosen to measure the fatigue strength of the material. We have performed an experimental campaign on 15 specimens extracted from a die cast part realized with the same process and with the same material adopted for the flange. The extrapolation of the fatigue strength has been executed with the staircase approach, and the fatigue calculations on the flange have been performed with the method proposed by Von Mises and modified by Juvinall. The analyses have shown that the stress field acting on the flange is lower than the fatigue strength of the material, with a

reliability of $75 \%$. 



\section{List of figures}

Figure 1.1: Cost build up in the life cycle of a product ........................................... 10

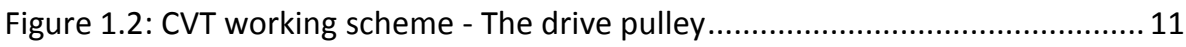

Figure 1.3: CVT working scheme - The driven pulley.......................................... 12

Figure 2.1: Typical CVT belt cross section............................................................. 13

Figure 2.2: Toothed shape of a typical CVT belt ..................................................... 14

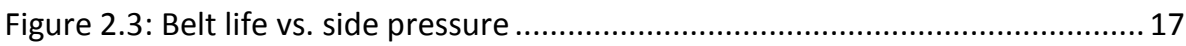

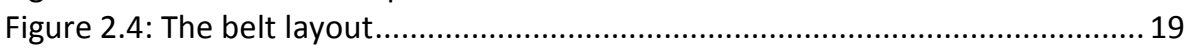

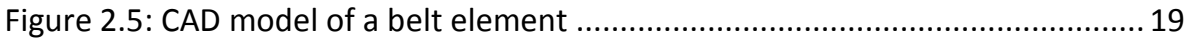

Figure 2.6: Traction test ............................................................................ 20

Figure 2.7: The force-displacement diagram obtained with the traction test ......... 21

Figure 2.8: Bending test................................................................................... 22

Figure 2.9: The bending moment vs. angular displacement in the bending test ..... 23

Figure 2.10: The stress field acting on the upper cushion...................................... 25

Figure 2.11: The stress field acting on the matrix .............................................. 26

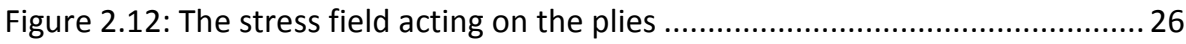

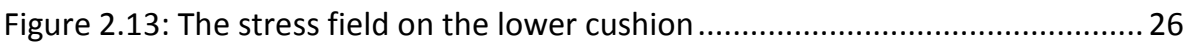

Figure 3.1: The hitting body discretization ......................................................... 30

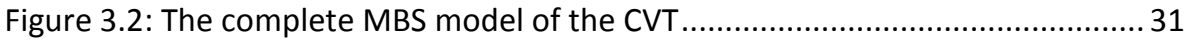

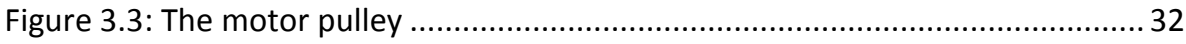

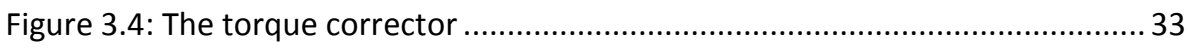

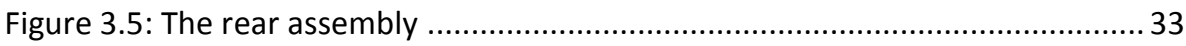

Figure 3.6: The model used to define the contact stiffness ................................. 34

Figure 3.7: Contact normal force distribution .................................................... 35

Figure 3.8: Force-Displacement behavior with a lateral force .............................. 36

Figure 3.9: Calibration of the elastic part of the contact formulation ...................... 37

Figure 3.10: Belt tension in the maximum torque test ....................................... 39

Figure 3.11: Contact force on the motor pulley in maximum torque conditions..... 40

Figure 3.12: The compression work done by the pulleys to the belt ........................ 40

Figure 3.13: Contact force on the driven pulley (blue line), angular velocity of the pulley (red line) and of the belt element (green line) in the maximum torque test 41

Figure 3.14: Belt tension in unsteady conditions (maximum torque) ...................... 43

Figure 3.15: Side contact force in unsteady conditions (maximum torque) ............ 43

Figure 3.16: Belt tension in the maximum power test ...................................... 45 
Figure 3.17: Contact force acting on the motor pulley under maximum power

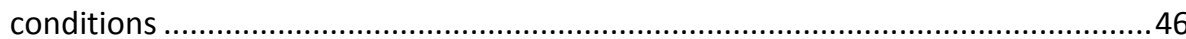

Figure 3.18: Contact force acting on the driven pulley under maximum power

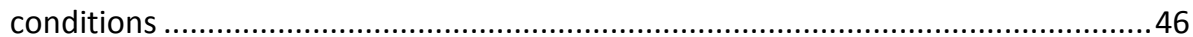

Figure 3.19: Belt tension in the over speed test ...................................................... 48

Figure 3.20: Contact force acting on the motor pulley under over speed conditions

49

Figure 3.21: Contact force acting on the driven pulley under over speed conditions

Figure 4.1: A typical CVT pulley construction .........................................................53

Figure 4.2: Axial section of the CVT half - pulley. Note the fins on the flange..........54

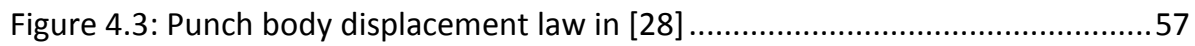

Figure 4.4: The riveting model described in [30] ...............................................58

Figure 4.5: Typical location of cracks in the half-pulley assembly ...........................59

Figure 4.6: The 18 degrees portion of the elementary set of the half-pulley............60

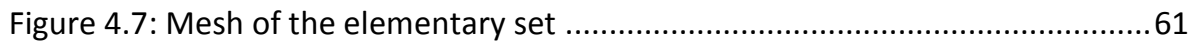

Figure 4.8: The model completely meshed .......................................................61

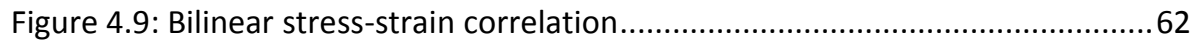

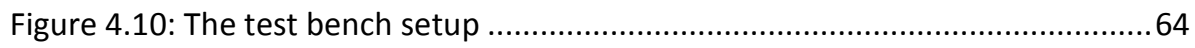

Figure 4.11: The static traction test performed on the flange material ..................65

Figure 4.12: An example of the definition of the contact elements..........................66

Figure 4.13: The force controlled punch body actuation ..........................................67

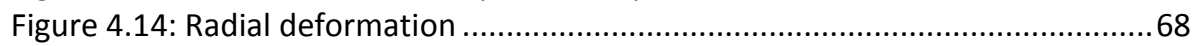

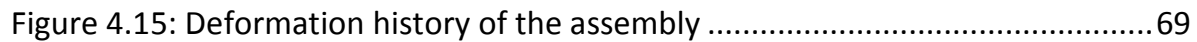

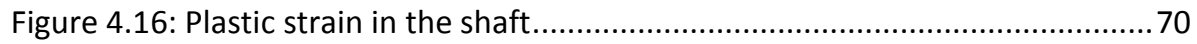

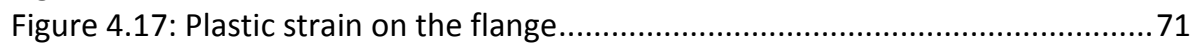

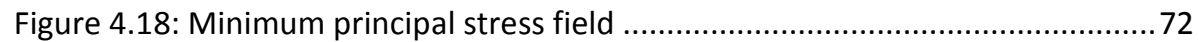

Figure 4.19: Minimum principal stress field in the shaft. The measure of " $z$ " points out the distance of the section plane from the surface of contact with the flange. 73 Figure 4.20: Minimum principal field in the flange. The measure of " $z$ " points out the distance of the section plane from the surface of contact with the flange. ......74

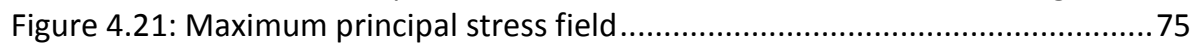

Figure 4.22: Maximum principal stress field in the shaft.........................................76

Figure 4.23: Maximum principal stress on the shaft .............................................. 77

Figure 4.24: Maximum principal stress field in the flange.........................................78

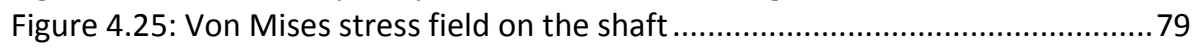

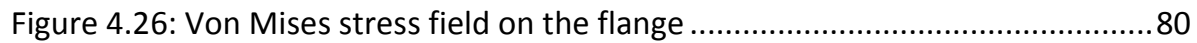

Figure 5.1: Magnified view of the failing surface of the specimen ............................83

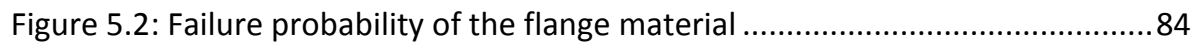

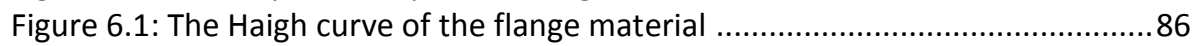

Figure 6.2: Graphical meaning of the equation (6.3) ........................................... 87 


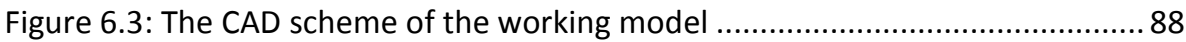

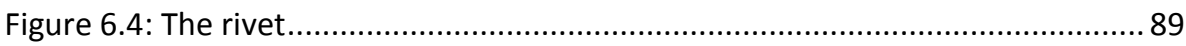

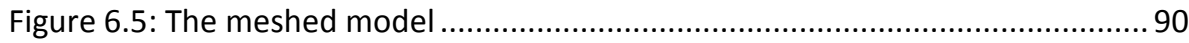

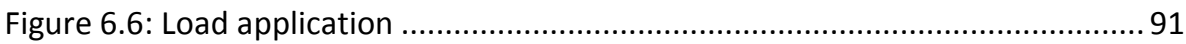

Figure 6.7: The full riveting residual stress ....................................................... 92

Figure 6.8: Maximum principal stress field on the flange ...................................... 93

Figure 6.9: Contact force in maximum torque conditions......................................94

Figure 6.10: Von Mises stress field in maximum torque conditions ........................ 95

Figure 6.11: Equivalent alternate stress field in the maximum torque conditions .. 95

Figure 6.12: Probability of failure in maximum torque conditions ......................... 96

Figure 6.13: Contact force in maximum power conditions .................................... 97

Figure 6.14: Von Mises stress field in maximum power conditions ......................... 98

Figure 6.15: Equivalent alternate stress field in the maximum power conditions... 98

Figure 6.16: Probability of failure in maximum power conditions ......................... 99

Figure 6.17: Contact force in over speed conditions .......................................... 100

Figure 6.18: Von Mises stress field in over speed conditions................................. 101

Figure 6.19: Equivalent stress field in the over speed conditions .......................... 101

Figure 6.20: Probability of failure in over speed conditions................................. 102 



\section{Bibliografy}

[1] Centro Studi Confindustria, "Quaderni di ricerca - Produttività e attrattività del Paese: i nodi da sciogliere", 2006.

[2] Centro Studi Confindustria, "Cambiare per crescere - La performance dell'Italia nel contesto internazionale", 2008.

[3] G. Don Taylor, "Design for global manufacturing and assembly", vol. 29, pp. 585597, 1997.

[4] D. Brazier and M. Leonard, "Participating in better decisions", Mechanical Engineering, Vol. 112, pp. 52-53, 1990.

[5] S. Dowlatshahi, "Product design in a concurrent engineering environment: an optimization approach", International Journal of Production Research, Vol. 30, pp. 1803-1818, 1992.

[6] Nilabh Srivastava and Imtiaz Haque, "A review on belt and chain continuously variable transmissions (CVT): Dynamics and control", Mechanism and Machine Theory, vol. 44, pp. 19-41, 2009.

[7] Riccardo Testi, Luigi Arnone, and Maurizio Marcacci, "CAE analysis of a CVT toothed belt", in SETC, Kyoto, Japan, 2002.

[8] Dae-Sung Bae and Edward J. Haug, "A recursive formulation for constrained mechanical system dynamics: Part II. Closed loop systems", Mechanics of structures and machines, vol. 15, no. 3, pp. 359-382, 1987.

[9] Dae-Sung Bae, Ruoh-Shih Hwang, and Edward J. Haug, "A recursive formulation for real time dynamic simulation of mechanical systems", Journal of Mechanical Design, vol. 113, pp. 158-166, Giugno 1991. 
[10] Daesung Bae, Heuije Cho, Seounghwan Lee, and Wonkyu Moon, "Recursive Formulas for design sensitivity analysis of mechanical systems", Computer methods in applied mechanics and engineering, no. 190, pp. 3865-3879, 2001.

[11] Wan-Suk Yoo, Sung-Soo Kim, Tae-Won Park, Dae-Sung Bae, and Jin-Hwan Choi, "Multibody dynamics research in Korea", JSME International Journal, vol. 46, no. 2, pp. 449-458, 2003.

[12] Werner Schiehlen, "Computational dynamics: theory and applications of multibody systems", European Journal of Mechanics A/Solids, no. 25, pp. 566594, 2006.

[13] A. Reungwetwattana and S. Toyama, "An Efficient Dynamic Formulation for Multibody Systems", Multibody system dynamics, no. 6, pp. 267-289, 2001.

[14] J. H. Critchley and K. S. Anderson, "A generalized recursive coordinate reduction method for multibody system dynamics", International Journal for Multiscale Computational Engineering, no. 1, pp. 181-199, 2003.

[15] John J. McPhee and Scott M. Redmond, "Modelling multibody systems with indirect coordinates", Computer methods in applied mechanics and engineering, no. 195, pp. 6942-6957, 2006.

[16] Jae-Hwan Lim, Hong Jae Yim, Si-Hyung Lim, and Taewon Park, "A study on numerical solution method for efficient dynamic analysis of constrained multibody systems", Journal of Mechanical Science and Technology, no. 22, pp. 714-721, 2008.

[17] Inc. FunctionBay. (2005) RecurDyn / Solver theoretical manual.

[18] Byungok Roh, Hosung Aum, Daesung Bae, Heuije Cho, and Hakyung Sung, "A relative contact formulation for multibody system dynamic", KSME International Journal, vol. 14, no. 12, pp. 1328-1336, 2000.

[19] Daniele Dettori, Francesco Frendo, Emilio Vitale, Luca Carmignani, and Riccardo Testi, "Simulazione dinamica di una trasmissione CVT per motorscooter tramite codice multibody", in Atti del XXXII Convegno AIAS, Salerno, 2003.

[20] T. Ide and H. Tanaka, "Contact force distribution between pulley sheave and metal pushing V-belt", in Proceedings of CVT 2002 Congress, 2002, pp. 343355.

[21] J. H. Park and S. N. Atluri, "Fatigue Growth of Multi-Cracks Near a Row of Fastener-Holes in a Fuselage Lap-Joint", Computational Mechanics, vol. 13, pp. 189-203, 1993.

[22] R. P. Müller, "An Experimental and Analytical Investigation on the Fatigue Behavior of Fuselage Riveted Lap Joints", PhD Thesis, Delft University of Technology, 1995. 
[23] B. Langrand, L. Patronelli, E. Deletombe, E. Markiewicz, and P. Drazétic, "Full scale experimental characterisation for riveted joint design", Aerospace Science and Thecnology, no. 6, pp. 333-342, 2002.

[24] T. J. Fitzgerald and J. B. Cohen, "Residual stresses in and around rivets in clad aluminum alloy plates", Material Science and Engineering, no. A188, pp. 51-58, 1994.

[25] T. B. Ryzhova, "Estimation of the reliability of ultrasonic quality control of riveted joints with clearance", Russian Journal of Nondestructive Testing, vol. 30, pp. 418-421, 1994.

[26] W. J. Slater, Static Strength of Riveted Joints in Fiber Metal Laminates, PhD Thesis ed.: Delft University of Technology, 1994.

[27] M. P. Szolwinski and T. N. Farris, "Linking Riveting Process Parameters to the Fatigue Performance of Riveted Aircraft Structures", Journal of Aircraft, vol. 37, no. 1, pp. 130-137, 2000.

[28] V. Blanchot and A. Daidie, "Riveted Assembly Modelling: Study and Numerical Characterisation of a Riveting Process", Journal of Materials Processing Technology, no. 180, pp. 201-209, 2006.

[29] A. Atre, "A Finite Element and Experimental Investigation on the fatigue of Riveted Lap Joints in Aircraft Applications", Georgia Institute of Technology, 2006.

[30] A. Del Grande and R. Testi, "FEM analysis of a CVT pulley", in International ANSYS Conference, 2006.

[31] Piaggio \& C. S.p.A., "Norma 2352", 1998.

[32] Tribology. [Online]. www.roymech.co.uk

[33] N. E. Dowling, "Mechanical Behavior of Materials", Prentice-Hall International Editions, 1993.

[34] R. C. Juvinall and K. M. Marshek, "Fondamenti della progettazione dei componenti delle macchine", Edizioni ETS, 1993. 